Florida International University FIU Digital Commons

3-30-2017

\title{
Using the Cognitive Interview to Enhance Recall During Contact Tracing
}

Alexandra E. Mosser

Florida International University, alimosser61@gmail.com

DOI: $10.25148 /$ etd.FIDC001801

Follow this and additional works at: https://digitalcommons.fiu.edu/etd

Part of the Cognitive Psychology Commons

\section{Recommended Citation}

Mosser, Alexandra E., "Using the Cognitive Interview to Enhance Recall During Contact Tracing" (2017). FIU Electronic Theses and Dissertations. 3176.

https://digitalcommons.fiu.edu/etd/3176

This work is brought to you for free and open access by the University Graduate School at FIU Digital Commons. It has been accepted for inclusion in FIU Electronic Theses and Dissertations by an authorized administrator of FIU Digital Commons. For more information, please contact dcc@fiu.edu. 


\section{FLORIDA INTERNATIONAL UNIVERSITY \\ Miami, Florida}

\section{USING THE COGNITIVE INTERVIEW TO ENHANCE RECALL DURING CONTACT TRACING}

A dissertation submitted in partial fulfillment of the requirements for the degree of

DOCTOR OF PHILOSOPHY

in

PSYCHOLOGY

by

Alexandra Mosser 
To: Dean Michael R. Heithaus

College of Arts, Sciences and Education

This dissertation, written by Alexandra Mosser, and entitled Using the Cognitive Interview to Enhance Recall During Contact Tracing, having been approved in respect to style and intellectual content, is referred to you for judgment.

We have read this dissertation and recommend that it be approved.

$\begin{array}{r}\hline \text { Mary Jo Trepka } \\ \hline \text { Lindsay Malloy } \\ \hline \text { Ronald Fisher } \\ \hline \text { Jacqueline Evans, Major Professor }\end{array}$

Date of Defense: March 30, 2017

The dissertation of Alexandra Mosser is approved.

Dean Michael R. Heithaus
College of Arts, Sciences and Education

College of Arts, Sciences and Education

Andrés G. Gil

Vice President for Research and Economic Development and Dean of the University Graduate School

Florida International University, 2017 
(C) Copyright 2017 by Alexandra Mosser

All rights reserved. 


\section{DEDICATION}

This dissertation is dedicated to the women who have inspired me, my family who has supported me, but most of all for Reid. 


\section{ACKNOWLEDGMENTS}

Thank you, first and foremost, to my advisor, Jacki Evans, for her endless feedback and support during the dissertation process. I never took for granted the obvious care, time, and effort you put into fostering the best project possible. I'm sure many, many students for years to come will benefit, as I have, from your mentorship. To the rest of my committee, your guidance has been invaluable. Ron Fisher, through your mentorship, humor and general chutzpah I learned to think both intuitively and critically, and, of course, to question everything. Lindsay Malloy, you encouraged me to undertake this project and I will always be grateful for the example you set as a woman in science. Finally, Mary Jo Trepka, you never hesitated to answer any questions I had regarding a field for which I knew little, and undoubtedly increased the quality of the work.

A huge thank you to my research assistants for their tireless efforts to get this work completed. I couldn't have done it without you. Thanks especially to Zoila Boza, Lazaro Nunez, Stephanie Padron, and Cynthia Weber. Thank you also to FIU's Department of Psychology, which supported this work through the Psychology Dissertation Year Fund and the Graduate Student Seed Fund Award.

Finally, Mom and Dad, you always told me that my best effort would be enough. I hope this work makes you proud. Blake and Michael, thank you for always letting me know you were in my corner. Lee and Cathy, thank you for your words of encouragement and support- it meant the world to me. Allison, thanks for getting me through the final push. Melissa, you made grad school worth it. You got me through some of the toughest days (and we had a lot of fun doing it). Finally, Reid, you know how I feel. This is for you, and I couldn't have done it without you. 


\title{
ABSTRACT OF THE DISSERTATION \\ USING THE COGNITIVE INTERVIEW TO ENHANCE RECALL DURING \\ CONTACT TRACING
}

by

\author{
Alexandra Mosser \\ Florida International University, 2017 \\ Miami, Florida \\ Professor Jacqueline Evans, Major Professor
}

To stem the spread of infectious diseases, epidemiologists use contact tracing interviews to identify individuals who may need treatment or, if indicated, quarantine or isolation. Given the high stakes, the most exhaustive list of potentially infected contacts must be reported. However, standard contact tracing procedures may fail to extract the most complete report possible from sick individuals. One of the most reliable methods for maximizing recall is the Cognitive Interview (CI). The CI uses several techniques grounded in psychological theory and was expected to increase the number of contacts listed during contact tracing interviewing compared to a standard contact tracing interview.

In Study One, participants imagined they were infected with meningococcal meningitis, and reported every person with whom they had physical contact, shared saliva, or lived with over the previous three days (i.e., at a high risk for developing meningococcal meningitis). Participants were interviewed with either a CI or a standard interview. Results suggested that the CI generated 35\% more total contacts listed, however, when examining only the contacts listed who would be at a high risk of 
meningococcal meningitis there was no significant difference between the CI and the standard interview.

Study Two followed the same procedure as that in Study One, but added a manipulation of cognitive resources intended to model impairment experienced by individuals who are interviewed while suffering from acute illness. Participants completed (or did not complete) a working memory impairment task (pressed a spacebar on a keyboard every time 7 seconds passed) while reporting their physical contacts during either a CI or a standard interview. Results clearly demonstrated a superiority of the $\mathrm{CI}$ in generating both more total contacts and more contacts at a risk of meningococcal meningitis than the standard interview. However, when the working memory impairment task was completed, the CI generated no more contacts than the standard interview. Findings have serious implications for contact tracing interviewing for infectious diseases such as Ebola and Zika. In light of the findings, we recommend the $\mathrm{CI}$ be considered as an alternative to the typical contact tracing interview, particularly if the interviewee is not cognitively impaired. 


\section{TABLE OF CONTENTS}

CHAPTER

PAGE

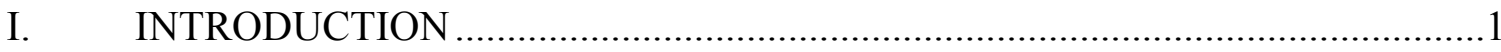

Contact Tracing.....

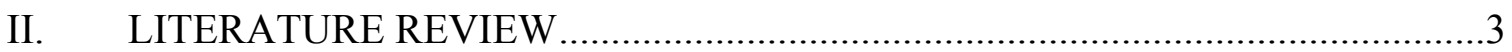

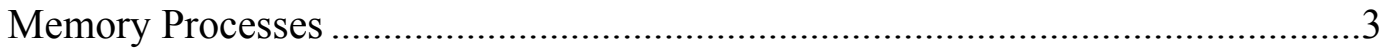

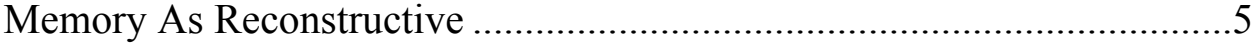

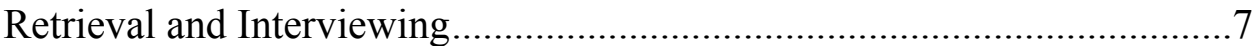

Retrieval Cues and Forgetting ................................................................

Food Histories and Sexual Contact Tracing …………......................................10

Food Histories ....................................................................................

Sexual Contact Tracing ........................................................................12

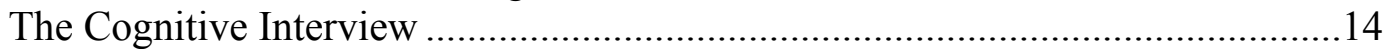

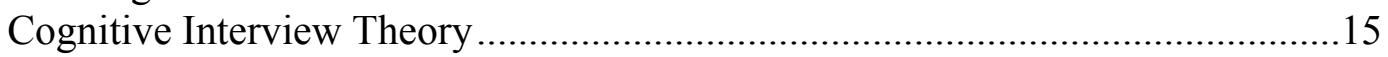

Basis of Social Dynamics in Psychological Theory ...................................15

Basis of Enhancing Cognitive Processes in Psychological Theory ...........19

Basis of Facilitating Communication in Psychological Theory .................21

Laboratory and Field Success of the Cognitive Interview....................................22

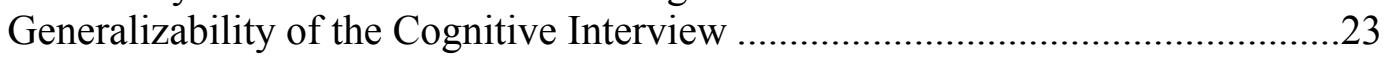

Illness and Cognitive Impairment ................................................................25

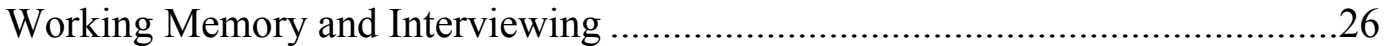

Cognitive Impairment and the Cognitive Interview ………...............................28

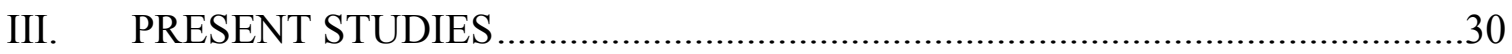

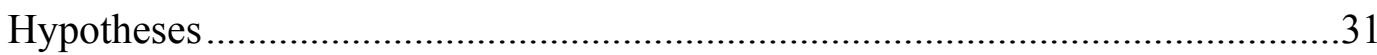

Study One

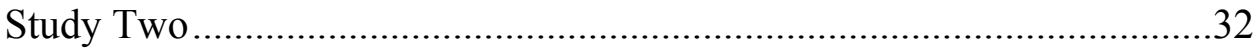

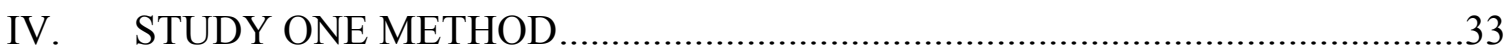

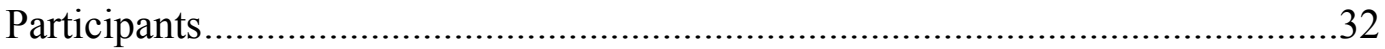

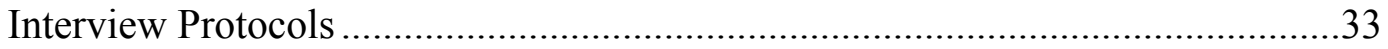

Standard Interview Protocol ...................................................................33

Cognitive Interview Protocol .....................................................................34

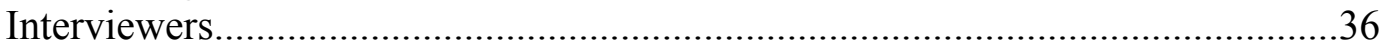

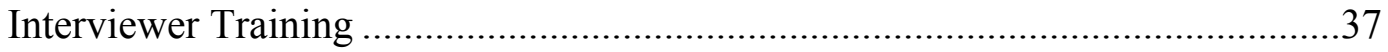

Standard Interview Training ..................................................................37

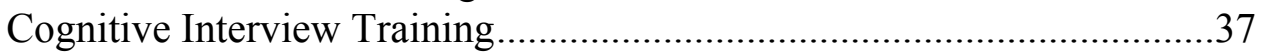

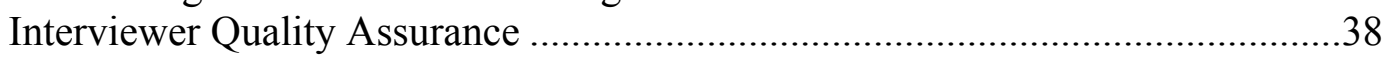

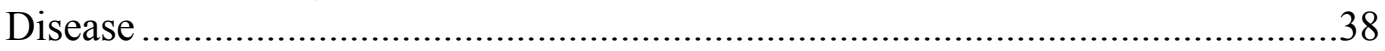

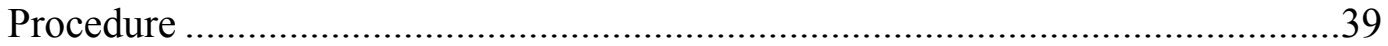

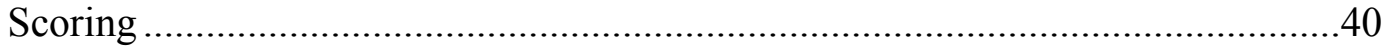


Scoring for Quantity …………………………………........................41

Scoring for Precision and List Utility .......................................................42

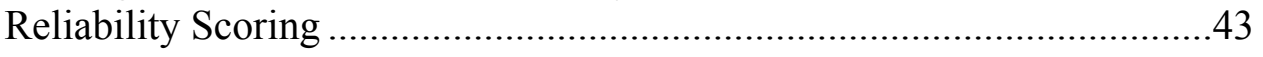

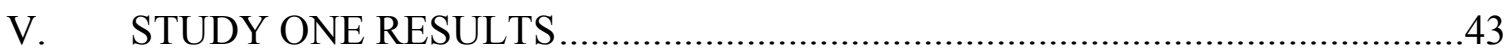

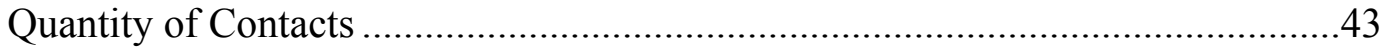

Average Precision of Contacts.....................................................................4

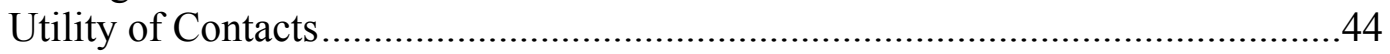

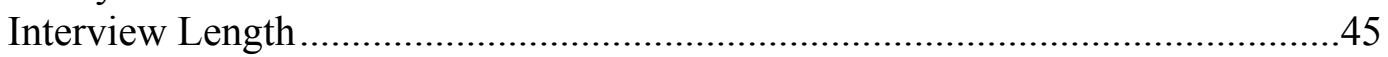

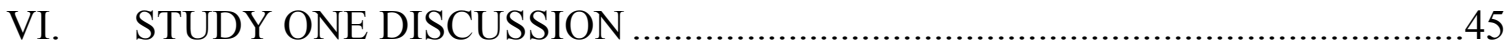

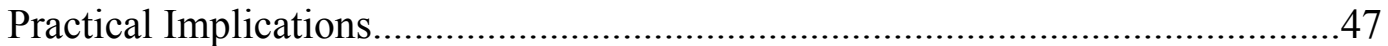

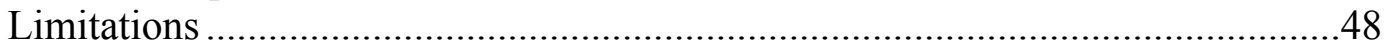

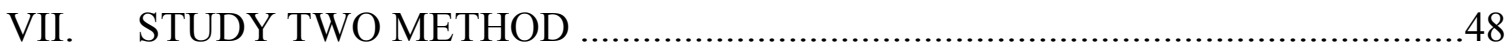

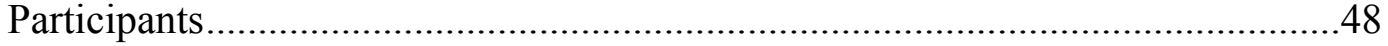

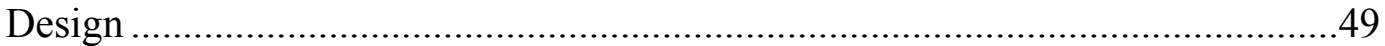

Interviewers and Interviewer Protocols ............................................................49

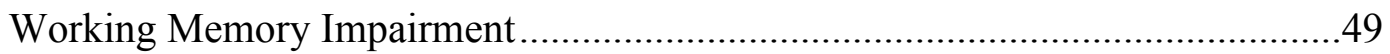

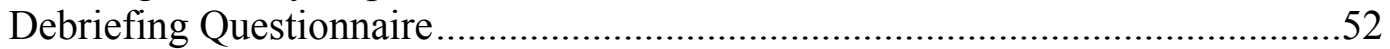

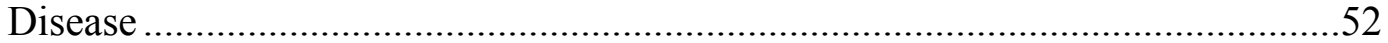

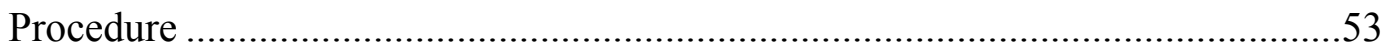

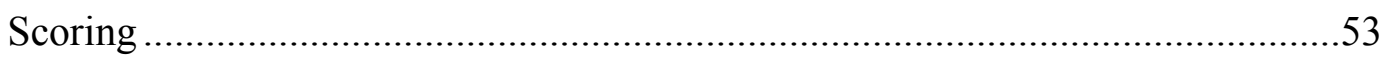

Scoring for Quantity, Precision, Utility, and Inter-Rater Reliability ..........54

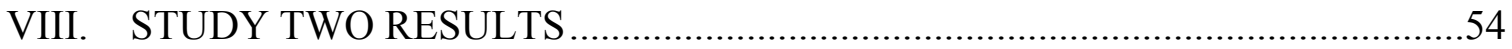

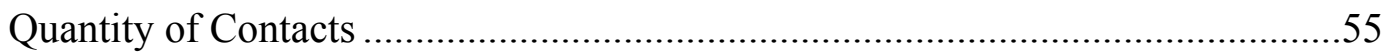

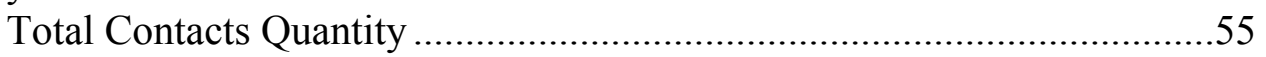

Droplet-Transmitted Quantity................................................................56

Average Precision of Contacts........................................................................57

Total Contacts Average Precision..........................................................57

Droplet-Transmitted Average Precision .................................................58

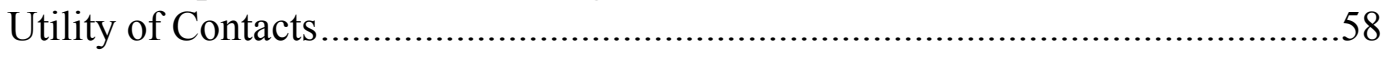

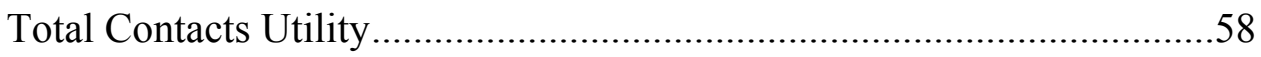

Droplet-Transmitted Utility ..............................................................59

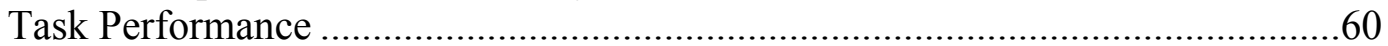

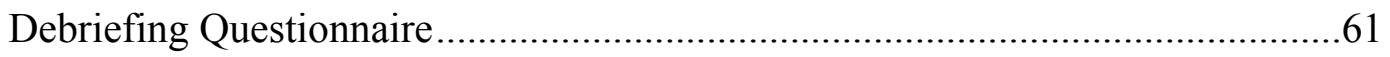

Subjective Assessments of Interview Experience........................................61

Subjective Assessments of Task Experience ………………....................62

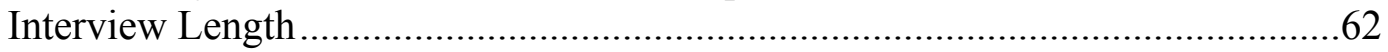

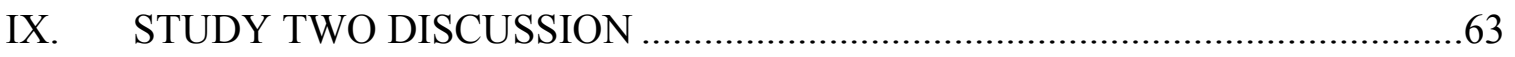

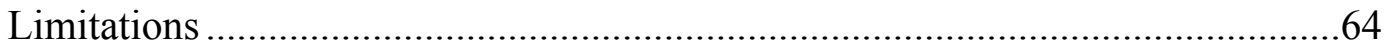

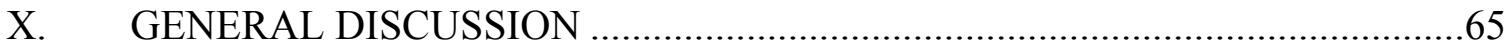




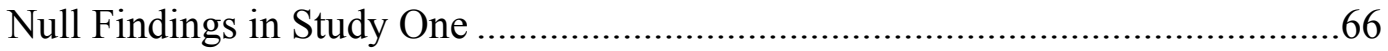

Novel Additions to CI Literature .....................................................................69

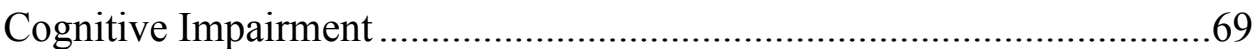

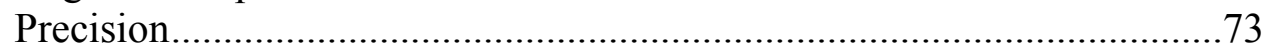

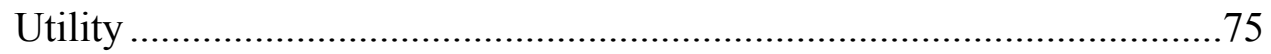

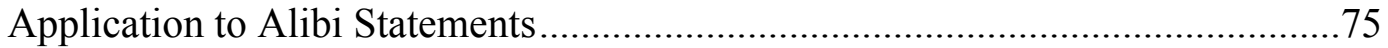

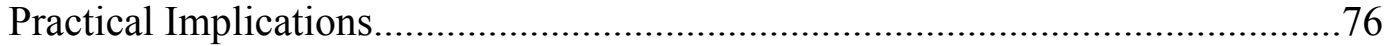

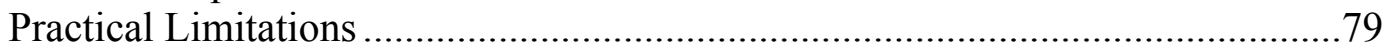

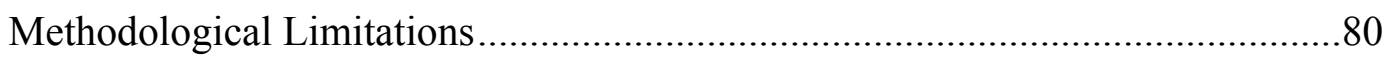

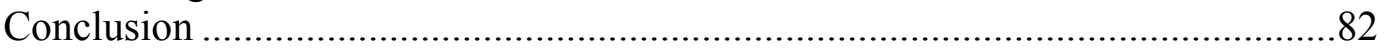

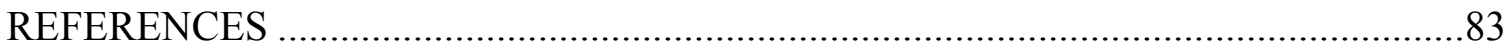

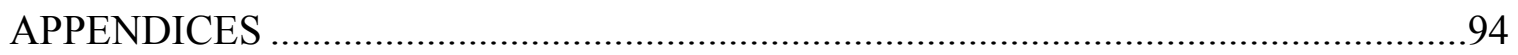

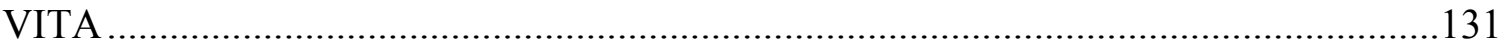




\section{LIST OF TABLES}

TABLE

PAGE

1. Study 2: Means, Standard Deviations and Confidence Interals of Total Contacts as a Function of Interview Type and Presence of Impairment Task

2. Study 2: Means, Standard Deviations and Confidence Intervals of DropletTransmitted Contacts As a Function of Interview Type and Presence of the Impairment Task

3. Study 2: Means, Standard Deviations and Confidence Intervals of Subjective Assessments of Difficulty Remembering, Mental Resources Expended, and Success at Remembering as a Function of Interview Type and Presence of the Impairment Task

4. Study 2: Means, Standard Deviations and Confidence Intervals of Subjective Assessments of Difficulty Completing Task, and Percent of Mental Resources Allocated to the Task as a Function of Interview Type

5. Study 2: Means, Standard Deviations and Confidence Intervals of Length of Interview as a Function of Interview Type and Presence of the Impairment Task ...123 


\section{LIST OF FIGURES}

FIGURE

PAGE

1. Study 2: Total Contacts listed as a function of interview type and presence of impairment task

2. Study 2: Droplet-transmitted contacts listed as a function of interview type and presence of impairment task

3. Study 2: Utility of total contacts as a function of interview type and presence of impairment task

4. Study 2: Utility of droplet-transmitted contacts as a function of interview type and presence of impairment task

5. Study 2: Ratings of difficulty remembering as a function of interview type and presence of impairment task (higher score indicates greater difficulty)

6. Study 2. Ratings of success of remembering contacts as a function of interview type and presence of impairment task (higher scores indicate greater success)

7. Study 2: Interview length as a function of interview type and presence of impairment task 


\section{INTRODUCTION}

Each year, tens-of-millions of lives are claimed by infectious diseases worldwide (CDC, 2014). Annual fatalities, however, can be substantially increased by devastating outbreaks such as that of Ebola in 2014, which led to an estimated 10,000 deaths (Sifferlin, 2015). To prevent accumulating casualties in this and other outbreaks, the World Health Organization (WHO) and the Centers for Disease Control and Prevention (CDC) use critical procedures to identify individuals for quarantine (for exposed but not

yet sick people) or isolation (for exposed and sick people). The following studies applied established research on memory and interviewing to these procedures with the goal of helping contain the spread of deadly infectious diseases.

\section{Contact Tracing}

The chief procedure used by the CDC to control several infectious diseases is contact tracing. Contact tracing is the practice of identifying and isolating individuals who have come in contact with infectious parties. Integral to the contact tracing process is asking infectious individuals to list both the people encountered and the places visited since the time of infection (Eames \& Kneeling, 2003). For example, imagine that a sick individual is admitted to a hospital for treatment. During questioning at intake, the medical staff learns that the individual has recently traveled from a region in which an Ebola outbreak occurred. As suspected, the individual tests positive for Ebola. Once the patient's immediate needs are met, the biggest concern for the medical staff becomes identifying whom may have been exposed to the patient when he or she was infectious and whom may have infected the patient. To investigate the patient's contacts, a contact tracing interview is conducted. The interview process begins by determining when the 
individual became contagious. Establishing the likely period of contagion allows the interviewer (typically an epidemiologist) to pinpoint the critical timeframe during which all contacts must be reported. Once the probably contagious period is identified, the epidemiologist asks the patient to recall every contact from contagion until entering the emergency room (by using anywhere from one to several open-ended prompts and follow-up questions; see Appendix A for the form completed in the Ebola outbreak). The listed contacts are subsequently investigated, and the relevant individuals are assessed for possible illness, quarantined if indicated, interviewed about their potential contacts, and ultimately treated, if ill.

Worldwide, contact tracing is paramount for controlling the spread of rare, but deadly infections. For example, outbreaks of severe acute respiratory syndrome (SARS) (Lipsitch et al., 2003), foot-and-mouth-disease (Kiss, Green \& Kao, 2005), smallpox (Porco, Holbrook, Fernyak, Portnoy, Reiter \& Aragon, 2004), avian influenza (Wu, Riley, Fraser \& Leung, 2006; cited in Armbruster \& Brandeau, 2007), and most recently Ebola virus disease (EVD) (CDC, 2014) are monitored using contact tracing procedures. Within the United States, more specifically, contact tracing is used to control the spread of low-prevalence infections such as tuberculosis (TB) (CDC, 2000), human immunodeficiency virus (HIV) (CDC, 2002), and various other sexually transmitted infections (STIs) (Clark, 1998; Cowan, French \& Johnson, 1996; cited in Armbruster \& Brandeau, 2007). The specific disease and the way it spreads (e.g., airborne, droplet, fomite) dictates the type of contacts targeted during the interview. For example, in an outbreak of meningococcal meningitis, which is spread through droplet-transmitted contact, patients would be asked to list the contacts whom they touched or shared saliva 
with; whereas in an outbreak of SARS, which can be transmitted through the airborne route, patients would be asked to list every person they may have encountered.

Contact tracing is vital to containing numerous deadly diseases for two reasons. First, it helps identify other infected people. These infected people will then be able to obtain treatment, and further transmission can be prevented either through counseling or, if indicated, by isolation until they are no longer infectious. Second, it helps identify exposed, and still healthy people. Symptoms can be monitored in these people, earlier treatment can be facilitated or in rare cases, such as an Ebola exposure, quarantine may be implemented until the incubation period has passed. Because it is imperative that every potentially exposed individual is identified (both to prevent further transmission and provide treatment), the most exhaustive list of contacts possible should be produced (e.g., Brewer et al., 2005; Eames \& Keeling, 2003; Potterat, 1997).

\section{LITERATURE REVIEW}

Despite its evidenced importance, surprisingly little research has examined the extent to which people can recall a comprehensive list of relevant contacts during contact tracing interviews. The minimal extant literature, however, disturbingly suggests that the typical contact tracing interview is gravely inadequate (e.g., Brewer, Garrett, \& Kulasingam, 1999). Fortunately, extensive research in the areas of cognition generally, and eyewitness memory specifically, can provide guidance on how the contact tracing interview can be improved.

\section{Memory Processes}

Recalling contacts is fundamentally a memory task. As such it is subject to the conventional principles and fallibilities of human memory. Indeed, extensive cognitive 
psychology research has demonstrated that during a simple listing task, such as that used during contact tracing when an interviewer simply requests a list of contacts, both errors of omission (i.e., forgetting) and errors of commission (i.e., false alarms) almost always occur (e.g., Anderson, Bjork \& Bjork, 1994; Krall \& Dwyer, 1987; Nelson, 1971; Tulving \& Osler, 1968; Tulving \& Pearlstone, 1966). Even more troubling, research has demonstrated that errors of omission and commission are often committed by witnesses recalling the people encountered (much like a contact tracing interview) over the course of a criminal event (e.g., Cohen \& Faulkner, 1989; Fisher \& Geiselman, 1992; MacLeod, 2002; Migueles \& Garcia-Bajos, 2007; Shaw, Bjork, \& Handal, 1995). To fully appreciate the fundamental difficulty of complete reporting during a contact tracing interview, it is first important to have a basic understanding of memory processes.

Memory is traditionally depicted as occurring in three phases: encoding, storage, and retrieval (Melton, 1963; Fisher \& Geiselman, 1992). At the first phase of memory, encoding occurs. During encoding the stimuli is perceived and a mental representation of the stimuli is formed. Once encoded, the representation must be held in storage, comprising the second phase of memory. At the third phase of memory, the representation of the stimuli is activated from storage and a conscious recollection of the stimuli is formed (retrieval).

For example, imagine a woman who encounters an intoxicated man being asked to leave a restaurant. She will encode, and store, some details of the event (e.g., parts of the conversation, the appearance of the man). When the manager contacts the witness for a detailed report of the event, she correctly retrieves and reports some of the encoded details as the remembered event (e.g., the man was yelling about the bad service). 
Assume that the witness attempts to remember all she can about the critical night. Although earnestly attempting to remember, her report likely omits some of the originally encoded information. Because the witness was simply asked to recall the event without the interviewer providing any aid in retrieval, some of details will likely be forgotten (Fisher \& Geiselman, 1992). As is discussed later there are techniques that can be used to help witnesses more successfully retrieve details of an event.

Memory As Reconstructive. One pervasive lay mischaracterization of cognitive processes is that memory of an event comprises an exact replica of the experienced event. Accordingly, recalling the event should merely entail passively accessing it, much like playing back a video recording. In reality, however, encoding, storage, and retrieval are complex and active processes. The recollection of an event is reconstructed by incorporating a multitude of factors, including but not limited to the actual event. Memory is therefore conventionally characterized as a reconstructive process during which many variables influence the final report, including the witness's mental state during the event, the context in which the event was encoded, the witness's knowledge of similar events, and many other factors (e.g., Brown \& Craik, 2000; Fisher \& Geiselman, 1992; Schacter, 1999; Schacter, Guerin \& Jacques, 2011; Vincente \& Brewer, 1993). For example, the restaurant witness's representation of the critical event might include her anxiety at the event, her expectations of how an intoxicated person behaves, and her discussion of the event with fellow restaurant patrons.

Because memory is not a carbon copy of the experienced event, reconstructive processes can result in errors of omission and commission (e.g., Bartlett, 1932/1995; Bartlett \& Burt, 1933; Bransford \& Johnson, 1973; Neisser, 1967, cited in Mitchell \& 
Johnson, 2000; Schacter, 1999). For instance, the witness's knowledge of alcohol's effect on motor impairment could lead to an erroneous statement that the intoxicated patron tripped while exiting the restaurant. In this way, the witnessed event is altered by the factors that interact with the representation at encoding, and these factors ultimately shape the accuracy and detail of the provided report.

How easily a detail is retrevied (i.e., was the detail recalled quickly and/or with minimial effort / mnemonic aid) is influenced by factors at all three stages of the memory process. At the encoding phase, ease of eventual retrieval is influenced by how much attention was paid to the event, the depth of processing involved, and the number of times a witness was exposed to the same information (e.g., Cepeda et al., 2006; Craik, Govoni, Naveh-Benjamin, \& Anderson, 1996; Craik \& Tulving, 1975; Scarborough, Cortese, \& Scarborough, 1977). For example, if the witness had been asked to describe the face of the intoxicated individual, who also happened to be the witness's close friend, it would be easier to retrieve the details of his face (due to repeated exposure and familiarity) than it would be if he was a stranger. At the storage phase, ease of retrieval can be influenced by the amount of time that passes between the encoding and retrieval phases (e.g., Ebbinghaus, 1885; Murre \& Dros, 2015). For example, if a year had passed between the witness viewing the intoxicated individual and being interviewed by the restaurant manager, she would likely remember his face with less ease than if she had been interviewed the next day. At the retrieval phase, the amount and strength of cues present at retrieval, and the amount of interference between memories for that particular event and a similar event, can influence the ease with which something is retrieved (e.g., Lindsay, Allen, Chan, \& Dahl, 2004; Moscovitch \& Craik, 1976; Tulving \& Watkins, 
1975). For example, if the witness was questioned in the same restaurant in which the event took place, external cues in the environement (e.g., seeing the hostess stand again), may make it easier for her to retrieve more details about the event than if she had been questioned at home.

Retrieval and Interviewing. Researchers have identified retrieval as the most laborious task in the memory process (Fisher \& Geiselman, 1992). Remarkably, longterm memory stores an unlimited amount of information with unlimited duration. To successfully retrieve an event, an individual must, therefore, search through billions of memories, many of which are similar to each other.

The effortful, and oftentimes-conscious retrieval process is the memory stage most likely to be influenced by interviewing procedures (compared to encoding and storage). During the encoding of events, witnesses are often unaware they will need to remember the event later and are thus are not intentionally encoding it (i.e., they use incidental encoding). Similarly, storage processes are primarily subconscious (Fisher \& Geiselman, 1992). As such, conscious efforts to improve the encoding and storage of personally experienced events are generally futile (although learning techniques can be successfully employed to facilitate encoding; e.g., Klein \& Kihlstrom, 1986; Pirolli and Anderson, 1985; Symons \& Johnson, 1997). In contrast, retrieval is an effortful process, often under conscious control (Klatzky, 1980; Fisher \& Geiselman, 1992). Retrieval has therefore been targeted for improvement via various memory techniques. Specifically, interview procedures aimed at enhancing recall, such as the Cognitive Interview (CI), tap into this potential by optimizing the retrieval phase of memory (Fisher \& Geiselman, 1992). 
Retrieval Cues and Forgetting. The CI attempts to improve retrieval by providing retrieval cues to overcome witness forgetting (i.e., errors of omission). Providing cues to prevent forgetting directly contradicts a common misconception that remembering depends exclusively on whether the encoded information is present in the memory store (Fisher \& Geiselman, 1992). Particularly common is the belief that if a fact is reported, then that fact must have been encoded and is therefore stored in memory, but if a fact is not reported, then that fact was not encoded and stored in memory (i.e., tracedependent forgetting). All forgetting cannot be accounted for by a lack of information in the memory store, however. Forgetting often occurs because of a lack of appropriate cues at retrieval (i.e., cue-dependent forgetting; e.g., Tulving, 1974). For example, the aforementioned witness might fail to report that the waiter had pushed the assailant during the critical exchange. The omission might be explained as a lack of effective retrieval cues for the waiter's actions. If she had thought about the waiter yelling, "Move aside!" she may have remembered that the waiter had pushed the assailant to get him out of the way.

Retrieval cues can be either internal or external. An internal cue is one generated by the witness's internal processes during retrieval (e.g., witnesses mood). The witness cuing herself to the waiter's push by thinking about his exclamation of "Move aside!" is an example of an internal cue. Conversely, an external cue is one prompted from outside forces in the environment (Fisher \& Geiselman, 1992). If the interviewer had asked the witness whether the waiter touched the assailant, or to describe the event from the waiter's perspective, it may have cued her to report the push. 
Notably, prolific cognitive researcher Endel Tulving (1974) conceptualized memory as being composed of both the original trace of the event, and the cues present in the environment during retrieval. To retrieve information from the memory store, the proper cue must be present (e.g., Tulving and Watkins, 1975). Thus, a seemingly "forgotten" memory (e.g., the waiter's push) can often be retrieved if the cue present at encoding is activated at retrieval (e.g., think about the event from the waiter's perspective or what the waiter said).

There is practical evidence that memories appearing to be "forgotten" are not lost forever and, in fact, can be retrieved given the right cues. For example, imagine a game show contestant asked to name all 50 states in one retrieval attempt. It is highly likely that at least one state will be forgotten (e.g., Oregon). Such an oversight does not suggest that the contestant does not know Oregon exists. Rather, it suggests a lack of retrieval cues reminding him to name it. For instance, if the contestant had been provided with a list of the different regions to consider (i.e., external cues), Oregon may have been listed.

The cue-dependent explanation of forgetting has been supported by decades of empirical work. A classic series of laboratory experiments (Tulving, 1974; Tulving \& Thompson, 1973) demonstrated that previously forgotten information could be remembered when retrieval cues were provided. For example, Tulving and Pearlstone (1966) presented participants with to-be-remembered words belonging to different categories (e.g., sports, flowers, animals). During recall, participants who were presented with the category name (serving as a retrieval cue) recalled significantly more words than those who were not. Researchers concluded that the participants who did not receive the 
category names lacked the appropriate retrieval cues, and thus suffered from a retrieval failure.

The evidenced difficulty of retrieval, as well as the ubiquity of cue-dependent forgetting (and consequently, the importance of cues at retrieval) has important implications for contact tracing interviewing. Notably, if a contact tracing interview involves merely asking an individual to list his or her contacts, the reviewed research suggests that a simple listing of stimuli will result in some forgetting. However, providing cues to retrieval can increase the amount of information generated.

\section{Food Histories and Sexual Contact Tracing}

To argue against the use of a conventional technique applied research related to contact tracing interviewing should be considered. Research on food histories and sexual contact tracing provides additional, robust evidence for the standard procedure's inadequacy. Both areas have found that individuals often provide insufficient and incomplete accounts when a standard procedure is used (Decker, Booth, Hutcheson \& Schaffner, 1986; Krall \& Dwyer, 1987; Mann, 1981).

Food Histories. During investigations of foodborne illness, epidemiologists interview individuals about the food they had consumed. These interviews help investigators pinpoint the specific food responsible for sickening a subset of the population. For example, food histories were used recently to trace various cases of $E$. coli and Salmonella to items from the popular Mexican fast food chain, Chipotle. To identify the offending food, the proportion of sick individuals who had consumed a certain food is compared to the proportion of healthy individuals who had also consumed 
that food. The food with the largest difference between the healthy and sick individuals is deemed a likely culprit (Mann, 1981).

Much like a typical contact tracing interview, in a food history interview an epidemiologist simply asks the individual to list all of the foods consumed during a period when the illness was likely contracted. For example, in one study, participants were asked about the foods they had consumed over a one-week period. To verify the comprehensiveness of the reports, participants filled out a daily food diary for that span of time. Results suggested that several foods initially written in participant diaries were commonly omitted in the list during the interview, indicating problems with forgetting during reporting (Krall \& Dwyer, 1987).

Another study employed different methods and similarly found the food history procedure to be inadequate. Mann (1981) invited participants to a controlled potluck meal at a research facility. Researchers monitored two dishes of interest and noted the individuals who had eaten each dish. Five days later participants were asked to report what they had eaten. Results demonstrated that participants both a) omitted foods they had actually consumed and b) reported eating target foods they had not consumed.

In almost all of the literature on reporting during food histories, authors have concluded that standard procedures should be improved. Specifically, using cognitive strategies to enhance recall has been acknowledged as a promising avenue for future research. In his review of health interviewing methodologies, Cannell (1970) emphasized that, "[the] standard questions may not represent the most adequate stimuli to activate respondent recall because they may ignore the way in which information is organized in memory" (cited in Mann, 1981). Other experts have also acknowledged the need to 
enhance the process by which memories are obtained in contact tracing interviews (cited in Fisher and Quigley, 1989).

In response to calls for improvement, Fisher and Quigley (1989) used a potluck paradigm to compare the standard food history interview to a well-established interviewing procedure, the Cognitive Interview (CI). The CI is a technique, which relies on techniques derived from classical principles of psychology to enhance individuals' recall during an interview (the $\mathrm{CI}$ will be described in detail later). To compare the contrasting interview techniques, 26 participants took part in a monitored potluck dinner. One week later participants were interviewed using either the standard food history interview or a modified version of the CI. Results demonstrated that more than two times as many foods (and with no loss in overall accuracy) were generated using the CI. This finding has two important implications. First, if more than twice as many foods were listed using a novel technique, there are obvious flaws with the standard questioning technique. Second, using an interview aimed at enhancing retrieval can substantially improve outcomes.

Sexual Contact Tracing. Mistakes made during food histories may have dire implications for the reporting of contacts during contact tracing. Although research on food histories has examined errors made while listing foods, rather than human contacts, arguably the same underlying memory principles are at work in both contexts. It is therefore plausible that the same types of errors demonstrated when reporting food histories are made when reporting individuals encountered during a contact tracing investigation. 
Brewer and colleagues $(1999,2001,2002 \& 2005)$ have, in fact, demonstrated similarities between the reporting of foods and human contacts. In their work, researchers examined the efficacy of sexual contact tracing in HIV investigations. When patients are admitted to an HIV clinic they are questioned about their sexual contacts as well as persons with whom they had shared needles. The contact tracing procedure allows for the notification and testing of the reported contacts.

Several studies have investigated a patient's ability to exhaustively report sexual contacts (Brewer, Garrett \& Kulasingam, 1999; Brewer, Garrett \& Rinaldi, 2002; Brewer, 2002; Brewer \& Garrett, 2001; Brewer et al., 2005). In a typical study Brewer and his colleagues asked patients infected with sexually transmitted diseases to, first, simply list their sexual and injection partners (replicating the typical questioning procedure). Researchers then asked repeatedly for more contacts by either simply asking the participant if there were any additional contacts he or she could list, or by using cognitive mnemonics to aid in recall (e.g., cue people to various places where it is likely to meet a partner, list a letter of the alphabet and ask participants to list names of any contacts that might begin with that letter, read back the already-remembered list of contacts to the participant). Repeated requests and/or the cognitive mnemonics caused patients to report substantially more contacts compared to the first open-ended request. Alarmingly, the standard procedure allowed for the forgetting of numerous sexual partners (accuracy could not be verified in this paradigm). In fact, in one study researchers estimated that up to $72 \%$ of sexual/injection partners listed were only remembered after repeated prompting (Brewer, Garrett, \& Kulasingam, 1999). Notably Brewer and colleagues (2005) concluded that omission errors during contact tracing were likely a result of a) the 
general forgetting of the contacts and b) the ignorance on the part of the patient that the interviewer wanted the most exhaustive list as possible. Research on sexual contact tracing has overwhelmingly demonstrated that the standard practice does not lead to a complete list of contacts. Thus, it is necessary to examine ways in which these methods could be improved.

\section{The Cognitive Interview}

One promising avenue for improving the standard contact tracing interviewing procedure is the CI. Originally developed by Ed Geiselman and Ronald Fisher for use in cooperative witness interviews, the CI implements established theories of social and cognitive psychology to increase the amount of information reported. The original CI, drawing on theories of encoding specificity and reminiscence, employs four specific mnemonics or memory aids to augment retrieval: mentally reinstating the emotional and physical context of the witnessed stimuli, changing the order in which the targeted information is reported (reverse-order), recalling the event from a different perspective (change-perspective), and an instruction to report exhaustively (e.g., Geiselman, 1984; Geiselman, Fisher, MacKinnon, \& Holland, 1986).

The original version of the CI was later modified to include important tenets of social psychology (the establishment of social dynamics and the enhancing of communication between the witness and interviewer). This most recent version of the CI employs three general components to aid in an individual's recall: a) establishing social dynamics; b) enhancing cognitive processes; and c) facilitating communication. The establishment of social dynamics includes the development of rapport, explicit instructions that the witness will do most of the talking, the use of open-ended questions, 
and avoiding interviewer interruptions. The cognitive processes of the witness are facilitated by reinstating the context (both physical and emotional) in which the event took place, asking for witnesses to repeatedly search through memory and approach the memory from different perspectives, asking non-suggestive questions, tailoring the questions to match the way the witness encoded the event, asking the witness not to guess, and instructing the witness to close his or her eyes during responding. Finally, the communication between the interviewer and the witness is enhanced by employing ways to convey information that may not be readily provided in a verbal form. Most notably, the sketch is recommended as a way to elicit spatially encoded information. Taken together, these tools should work to alleviate Brewer and colleagues' (2005) concerns regarding forgetting during a contact tracing interview by a) alleviating general forgetting and b) communicating to the witness that the interviewer wants the most exhaustive report as possible (Fisher, Milne, Bull, 2011; Fisher \& Geiselman, 1992).

\section{Cognitive Interview Theory}

The CI is heavily rooted in psychological theory. In fact, each of the CI's established techniques can be traced to classic psychological principles. The CI's reliance on evidence-based theory provides robust grounds for predicting an increase in contacts generated by a CI compared to a typical contact tracing interview.

Basis of Social Dynamics in Psychological Theory. The establishment of social dynamics was incorporated into the $\mathrm{CI}$ as a result of research suggesting that certain social techniques can optimize the amount of information gathered. For example, in the CI, interviewers are instructed to develop rapport by personalizing the interview (e.g., actively listening, using the witness's name) and communicating empathy (e.g., letting 
the witness know his or her feelings are understood) (Fisher \& Geiselman, 1992). The establishment of rapport has clear support in the psychological literature (Abbe \& Brandon, 2013; Collins, Lincoln \& Frank 2002; Kieckhaefer, Vallano \& Schreiber Compo, 2011; 2015). Positive working rapport has been noted to reduce the witness's anxiety at reporting, as well as establish trust between the parties. Oftentimes, during a contact tracing interview, the witness is reluctant to report because of the shame associated with having contracted the disease or a reluctance to confine friends and family to quarantine. Researchers have posited that positive rapport can increase trust and comfort at reporting, thereby increasing the number of contacts provided (e.g., Chapple, 1999).

The use of open-ended questions (e.g., "describe the robber") is another example of an empirically grounded technique, which influences not only social dynamics, but also the cognitive processes of the witness (Wright, Fisher \& Powell, 2004). The use of open-ended questioning has been touted as one of the most important recommendations for use in interviewing. In fact, an extensive body of literature suggests that witnesses tend to provide more information, and more accurate information in response to openended questions (Fisher et al., 2012). However, it is important to note that, as indicated by Fisher and colleagues (2012), it is difficult to compare the accuracy of closed-ended (e.g., "was the robber wearing a mask?") to open-ended questions (e.g., describe the robber?) because they often vary on other factors as well (e.g., differing levels of difficulty). Nevertheless, open-ended questions are primarily recommended to a) convey to the witness to provide a lot of information (rather than just responding to a few, 
pointed closed-ended questions) b) maximize meta-cognitive control, and c) reduce the chance of suggestion by the interviewer.

The use of open-ended questions has clear social value as well. When the interviewer asks only specific, closed-ended questions, it suggests to witnesses that they should wait for each question before generating a response. It also communicates that the only valuable information to be provided is the information that the investigator wants to know (e.g., whether the robber was wearing a mask or not). As a result, witnesses are reluctant to provide additional information because it is perceived as unimportant. The instruction for interviewers not to interrupt has related implications. If an interviewer continually interrupts the witness, the witness assumes that what he or she has to say is less important than any contribution made by the interviewer.

Much research also suggests that in a free-recall (open-ended) report, witnesses are able to maximize their metacognitive control. Koriat and Goldsmith (1996) posited a model whereby witnesses first monitor the accuracy of a response based on the subjective accuracy of the response (e.g., confidence, how fast it comes to mind). After the information is assessed for accuracy (metacognitive monitoring), if the subjective evaluation of accuracy passes the response threshold, the information is volunteered, otherwise it is withheld (metacognitive control). Research has suggested that witnesses are able to most effectively control the accuracy of their reports in a free report context (e.g., Evans \& Fisher, 2011). As such, it is expected that open-ended questions will lead to the most accurate reports, even as time passes and memory traces consequently weaken. 
In addition to maximizing control, open-ended questions also influence cognitive processing by allowing the witness to search through memory in a way that is compatible with how the event was encoded. Classic psychological literature refers to this as Transfer Appropriate Processing (TAP). When closed-ended questions are used, the witness recalls the event in the way specified by the interviewer's questions (rather than how it was initially encoded). Thus, open-ended questions are recommended.

The use of open-ended questions is also important because it helps control the amount of information the interviewer inadvertently leaks to the witness. Oftentimes closed-ended questions can become leading or suggestive. Suggestive questions can lead to the witness to report information that is inaccurate and based merely on information provided by the interviewer. The effects of post-event misinformation have been studied extensively, and suggest that the accuracy of witness reports is harmed by these intrusions (e.g., Loftus, 1975; Loftus \& Zanni, 1975; Roebers \& Schneider, 2000).

During the development of social dynamics witnesses are also explicitly instructed that they are the experts, know the most about the event, should not wait for questions to respond, and are in control of the interview. This is useful for multiple reasons. First, it helps to overcome some of the problems associated with a witness's preconceived notions about what a typical interview entails. Oftentimes a witness believes that the interview will be conducted much like seen on TV; the investigators will ask many skillful questions and will solve the case based on their masterful questioning technique. This instruction also informs witnesses that they will be doing a great deal of the talking. As a result, the witness is likely to provide more information than if not provided with this instruction. Finally, this instruction puts the witness in control of the 
reporting process. Therefore, witnesses should be more comfortable providing new information spontaneously throughout the interview.

Basis of Enhancing Cognitive Processes in Psychological Theory. The cognitive techniques of the CI are based largely on influencing the participant's retrieval processes. To aid in the fluent retrieval of the witnessed event, Fisher and Geiselman borrowed from several well-established theories of cognitive psychology. For example, one of the major tenets of the CI is to search through memory repeatedly. Research suggests that the more retrieval attempts that are made, the more likely new information will be provided (Roediger \& Payne, 1982). Indeed, much research has demonstrated that reminiscence (recalling an item at a second instance of retrieval that was not reported during the first) is common in repeated interviews (e.g., Gilbert \& Fisher, 2006; Hershowitz \& Turner, 2007; La Rooy, Pipe, \& Murray 2005; La Rooy, \& Lamb, 2011; Oeberst, 2012; Roediger, McDermott \& Groff, 1997; Turtle \& Yuille, 1994). For instance, in one study, every participant questioned about a mock crime made at least one reminiscent response (Oeberst, 2012). Gilbert and Fisher (2006) similarly reported that $98 \%$ of participants reminisced during a second retrieval attempt. In the CI, witnesses attempt retrieval multiple times, much like undergoing a second interview. These multiple attempts tend to lead to the addition of new details.

It has also been suggested that there are many different paths to retrieval (Anderson \& Prichert, 1978). For example, the aforementioned restaurant patron might struggle visualizing the intoxicated assailant's clothing, but then remember vividly the appearance of the assailant when thinking about the timbre of his voice. In line with this thinking, the CI encourages the witness to explore different means of retrieval. For 
example, witnesses may be asked to approach the memory through different perspectives. Classic research has demonstrated that when asked to adopt another person's perspective (e.g., report the event from the waiter's perspective) after an initial recall attempt, more information can be retrieved (Anderson \& Prichert, 1978).

Yet another way in which retrieval can be aided is through providing retrieval cues to the witness during reporting. In the CI these cues can be provided through context reinstatement. Related directly to the idea that cues are necessary for successful retrieval (i.e., cue-dependent forgetting), is the finding that memory is best when the context at encoding matches the context at retrieval (i.e., the Encoding Specificity Principle; e.g. Tulving and Thompson, 1973). In one famous experiment (Godden \& Baddeley, 1975) participants encoded a series of to-be-remembered information either on dry land (above water) or under water (scuba diving). Participants were then asked to recall the information either on dry land or under water. Results demonstrated that participants remembered the items better when they were encoded and recalled in the same context (either both under water or both above land).

In accordance with the literature regarding the Encoding Specificity Principle, Fisher and Geiselman incorporated context reinstatement into the CI as a tool to make the context at retrieval as close as possible to that during encoding. During contextreinstatement the witness is asked to mentally recreate the psychological, emotional, and/or physiological context of encoding (Fisher \& Geiselman, 1992). By matching (as close to possible) the context at encoding to the context at retrieval, the cues present at encoding of the event should also be present at retrieval. As a result, more information should be elicited from the witness than if context reinstatement is not used. 
The CI's instruction for witnesses to close their eyes during reporting is also based on classic theories of human cognition. The process of retrieval, especially as it pertains to the CI, is rather effortful. Witnesses are asked to extensively search their memories, requiring substantial concentration by the witness. As a result, the interviewer should facilitate concentration in any way possible. Instructing the witness to close his or her eyes is one of the Cl's proposed methods to enhance concentration. Closing of the eyes allows the witness to block out any external distractors and also allows for a more vivid mental image to be formed during retrieval (specifically during context reinstatement) (e.g., Vredeveldt et al., 2015; Vredeveldt, Baddeley \& Hitch, 2014; Vredeveldt, Hitch \& Baddeley, 2011; Vredeveldt \& Penrod, 2013; Vredeveldt \& Perfect, 2014; Vredeveldt \& Sauer, 2015).

\section{Basis of Facilitating Communication in Psychological Theory. The CI also}

aims to facilitate communication by providing ways for the witness to express information that might not be amenable to verbal form. The best example of facilitating communication in the $\mathrm{CI}$ is perhaps the sketch. Oftentimes witnesses struggle to verbalize certain aspects of an event. For example, it may be difficult to portray where certain parties were positioned, or how they moved about the space. In this instance the CI recommends the witness sketch the event or scene and narrate while sketching. Not only does the sketch itself provide more information about the event, but the act of narrating while sketching also serves as another retrieval attempt, resulting in the addition of new details. 


\section{Laboratory and Field Success of the Cognitive Interview}

Years of field and laboratory studies have comprehensively examined the efficacy of the CI under numerous conditions (for reviews see Fisher, Ross, \& Cahill, 2010; Griffiths \& Milne, 2010; for meta-analyses see Kohnken, Milne, Memon, \& Bull, 1999 and Memon, Meissner, \& Fraser, 2010). In a typical CI study, participants (college students) are asked to encode some to-be-remembered event (a live or videotaped event) that is criminal (e.g., bank robbery) or neutral (e.g., conversation between professor and student) in nature. After encoding the event, participants are interviewed by someone trained in either the CI or a standard interview commonly used in the field (e.g., Federal Law Enforcement's Five-Step Interview). Transcripts of the interviews are subsequently analyzed to assess differences in the amount and accuracy of the information gathered by the contrasting interviews. Hundreds of laboratory and field experiments have been conducted across the world and have explored many different variables including the type of witness (e.g., children, intellectually disabled, police officers), type of witnessed event (e.g., crime, accident, terrorist meeting), and delay between event and interview (e.g., immediately after, weeks after, 35 years after the event).

Previous research has overwhelmingly demonstrated that the CI substantially increases the amount of information gathered during an interview (Clifford \& George, 1996; Fisher, Geiselman \& Amador; Fisher, Milne, \& Bull, 2011; Fisher \& Schreiber, 2007; Kebbell \& Milne, 1998; Kebbell, Milne \& Flagstaff, 1999; Köhnken et al., 1999; Memon, 2006). In fact, across all published studies the CI tended to increase the amount of information gathered by anywhere from $25 \%$ to $50 \%$ compared to a standard interview (Memon, Meissner \& Fraser, 2010). In one field study alone, detectives trained on the CI 
gathered 63\% more information than untrained detectives (Fisher, Geiselman \& Amador, 1989).

Two notable meta-analyses have analyzed the combined effects of the CI across all available research. In an early meta-analysis of 36 studies conducted on the CI, researchers reported a large effect size for the increase in accurate information obtained by a CI, with a slight increase in inaccurate information gathered. Nevertheless, the CI was no less accurate proportionally than the control interview (i.e., the accuracy rates were equivalent; Kohnken, Milne, Memon \& Bull, 1999). In a more recent meta-analysis, Memon and colleagues (2010) similarly demonstrated a large increase in correct details, a small but significant effect of increase in incorrect details, and no significant increase in confabulated details.

\section{Generalizability of the Cognitive Interview}

The CI, supported by over 25 years of research, has been acknowledged as one of the most successful advances made in the field of law and psychology (Memon et al., 2010). As such, it has been applied to a wealth of contrasting areas in which individuals are interviewed for information. Furthermore, because the CI is based on general principles of memory and cognition (e.g., Tulving \& Thompson, 1973; Gilbert \& Fisher, 2006), it lends itself easily to any type of interview. For example, the CI has been applied to car accident investigations (Brock, Fisher, \& Cutler, 1999), epidemiological interviews about physical activity that occurred 35 years earlier (Fisher, Falkner, Trevisan, \& McCauley, 2000) and, of course, food history interviews (Fisher \& Quigley, 1992). We therefore expect the CI to translate readily to contact tracing. In Study One we tested 
whether a CI adapted for contact tracing increases the amount of information provided during a contact tracing interview compared to the standard interviewing procedure.

It is important to note that in contact tracing the value of a list of contacts lies in its quantity rather than accuracy. Thus, in any given contact tracing scenario, it is more important to have a very long list than to have a very accurate list. For example, if an infectious individual lists 15 potential contacts but 3 of them are false alarms, it is more advantageous than an infectious individual who lists 11 contacts, all of which are accurate. As such, a potential increase (compared to the standard interview) in inaccurate details caused by a CI is not a concern of the present studies. Rather, the present studies are a first step in evaluating whether the $\mathrm{CI}$ increases the number of contacts listed by individuals who are interviewed about their recent contacts.

Most interviewing research, including CI research, focuses on two measures of efficacy: quantity and accuracy. It is worth noting there is another relevant measure that has not received much attention: precision. Precision refers to the level of specificity with which a detail of an event is described (e.g., Evans \& Fisher, 2011). For example, if a witness describes the robber's shirt as "a navy polo with green stripes" the response would be considered more precise than if the witness describes the robber's shirt as "a dark polo with stripes". In the present studies we also assessed the effects of the CI versus a standard interview on a measure of precision: the ease with which a particular contact could be found as a result of the information provided. 


\section{Illness and Cognitive Impairment}

Although the CI has been readily applied to various types of interviewing, unique features of interviewing for contacts make this area of study novel. Critically, the individuals interviewed during a contact tracing interview are often acutely ill. Impairment of regular cognitive function is one of many deleterious side effects of infection. Laboratory research has identified several aspects of neurocognitive functioning associated with acute infection (for a review see Smith, 2013). Because patients interviewed during a contact tracing interview are ill, the effects of cognitive impairment on contact interviewing should be evaluated.

Smith (2012) evaluated the effects of acute infection on cognitive performance by first presenting participants with a series of cognitive tests to establish their baseline cognitive functioning. During a span of 90 days, a third of the participants returned to the lab after naturally developing a cold. The other two thirds of participants never became ill and returned to the lab as the control group. Illness was found to cause slower reaction times on cognitive tests, slower learning of novel information, as well as deficits (slower responses) in verbal reasoning and semantic processing compared to the healthy control group. Smith noted the failed transmission of noradrenaline (related to reaction times), choline (related to learning new information) and dopamine (related to working memory speed) as a cause of poor cognitive performance when ill.

Smith (2012) also compared a group of ill and healthy individuals on a driving task. Results suggested that being sick with a cold negatively impacted driving ability. Specifically, sick individuals were less likely to detect collisions and reacted more slowly to unexpected road obstacles than healthy individuals. 
More recently, Cvejic and colleagues (2014) presented a battery of neurocognitive tests to a cohort of participants infected with Epstein Barr virus, Ross River virus, or Coxiella burnetii. Participants were tested when ill, and again after obtaining complete recovery. Testing revealed that acute infection led to slower matching-to-sample responses, poorer working memory capacity, mental planning, and dual attention task performance, and longer time to complete discordant Stroop trials compared to recovery. Researchers concluded that the slower responses, as well as difficulty in completing complex tasks signaled acute impairment of neurocognitive functioning (particularly as it relates to the interference of related neurotransmitters). The impairment was especially associated with higher-order, executive functioning (working memory) and was noted as having potentially grave implications for completing every-day tasks when ill (e.g., remembering what you ate for lunch yesterday).

Generally, being ill impairs the types of executive cognitive functioning critical for retrieving information during an interview (i.e., working memory). As reviewed below, working memory affects almost every facet of retrieval. For any interviewing method to elicit the most extensive list possible from ill individuals, it must work for individuals with impaired working memory functioning. In Study Two, we sought to replicate and extend Study One by testing whether the CI improves recall compared to a standard interview for participants who are, or are not, cognitively impaired.

\section{Working Memory and Interviewing}

Working memory is an essential component of human cognition and is the avenue through which we are able to maintain information over short periods of time. For example, working memory allows an individual to remember the phone number of an 
attractive acquaintance from the bar, or the question just posed by the professor in front of the class. However, in contrast to the now passé "short-term" memory, working memory is understood as much more than just a short-term, limited capacity storage system (although both characterizations still apply to working memory). In addition to acting as a store, working memory also helps to process and retrieve learned information. For example, the author might use her working memory to remember in exactly which room she threw her car keys yesterday (that information is drawn from long-term memory into working memory and processed for conscious retrieval).

The complex conceptualization of working memory was originally proposed by Baddely and Hitch (1974) to include multiple systems (Central Executive, Phonological Loop, Articulatory Control and Visuo-Spatial Sketchpad), all of which work together to process and retrieve information. The central executive component $(\mathrm{CE})$, which is conceptualized as a command center, was the first system proposed by Baddeley and Hitch. Although the CE does not store information, it has direct access to long-term memory. With help from the CE, information moves backwards from long-term memory into working memory to complete certain tasks.

The CE also directs the three slave systems of working memory. The first is the phonological loop, which is composed of both the phonological store (inner ear; speech perception) and the articulatory loop. Known as the inner voice, for its speech production function, the articulatory loop is the system that silently repeats the phone number of the attractive person from the bar. The second slave system is the visuo-spatial sketchpad (VSS), also known as the inner eye. The VSS is used for visual and spatial tasks, including knowledge of where you are in a space. Finally, noticing a lack of capacity for 
the temporary storage of information in the working memory model, Baddeley (2000) added the episodic buffer as working memory's temporary storehouse where information stored in different modalities can be worked with together.

Working memory is undeniably important for both the processing and retrieval of information. As such, impairment of working memory has potentially severe consequences for performance during an interview. For example, a witness with impaired working memory may have difficulty maintaining focused attention for the length of the interview, keeping track of the questions the interviewer is asking and the names already provided during the interview, retrieving information from long-term memory, developing mental imagery, and generating internal retrieval cues, amongst many others.

\section{Cognitive Impairment and the Cognitive Interview}

Little is known about the CI's effects on cognitively impaired individuals. On the one hand it is reasonable to predict that the CI will be particularly effective for the cognitively impaired. The CI employs a host of social and cognitive retrieval aids and should, therefore, potentially help to overcome the impairment associated with illness. On the other hand, it is reasonable to predict that the CI will fail to improve recall in the cognitively impaired. The CI uses complex mnemonics and asks the participant to laboriously recall many different events in extreme detail (requiring full concentration and mental effort). Thus, the cognitively impaired may lack the resources required by the CI. Cognitive impairment might therefore lead to no increase in information gathered by the CI compared to the comparatively less demanding and less complex typical contact tracing interview. To help predict the CI's effects on the ill, we can look to a small body of research that has examined the effects of the CI on the intellectually disabled. 
Much like individuals who are cognitively impaired by illness, chronically intellectually disabled (ID) adults are slower to retrieve details and provide fewer details of an event than other, non-disabled adults (Milne \& Bull, 2001; Perlman, Ericsson, Esses \& Isaacs, 1994). Whereas sick individuals are likely only impaired at retrieval, ID adults are also impaired at the encoding and storage phases as well. Testimony of ID adults is invaluable to some cases. Thus, research has evaluated whether the CI increases the amount and accuracy of information provided by ID adults compared to a standard interview (for a review see Holliday, Brainerd, Reyna \& Humphries, 2009).

In an early study, Brown and Geiselman (1990) tested the effects of the CI versus a standard interview for witnesses to a to-be-remembered event who were either ID or non-disabled adults. Researchers found that ID adults provided fewer correct details, but significantly more confabulated details than the non-disabled adults. Notably, however, the CI generated more information than a standard interview regardless of whether participants were disabled or not.

In a second study, Milne and colleagues (1999) presented ID and non-disabled adults with a film of an accident. The next day participants were interviewed with either a $\mathrm{CI}$ or a structured control interview. Researchers encouragingly found that the CI led to an increase in correct details compared to the structured control interview; however, the CI also led the ID adults to generate more confabulated details (Milne \& Bull, 2001; Cited in Holliday, Brainerd, Reyna \& Humphries, 2009).

Recent research conducted by Wright and Holliday (2007) examined the efficacy of the CI on elderly adults with dementia. Elderly adults (ages 75-96) with and without dementia viewed a short film and were subsequently interviewed using a modified CI 
(omitting certain difficult elements), a full CI (no elements omitted), or a control interview. As expected, the participants with dementia reported fewer correct details than the healthy adults. However both CIs increased the amount of information recalled for participants with and without dementia compared to the control interview.

Taken together, the scant existing research suggests that the CI will increase the amount of information recalled compared to a standard interview for both cognitively impaired individuals and non-cognitively impaired individuals. Furthermore, the reviewed research seems to suggest that cognitive impairment at retrieval will decrease the amount of information provided compared to those who are unimpaired.

\section{PRESENT STUDIES}

The typical contact tracing method is still relied upon to stem the spread of deadly diseases despite its apparent weaknesses. Thus, it was necessary to evaluate ways in which the current procedure could be improved. In Study One participants were asked to report their contacts over the past 3 days using either a CI or a standard contact tracing interview. ${ }^{1}$ Study One was a first step in testing whether the well-established, evidencebased $\mathrm{CI}$ increases the number of contacts reported during a contact tracing interview compared to the standard interview.

To expand upon Study One, in Study Two some participants completed a second, distracting task (i.e., "a cognitive impairment task") during either a $\mathrm{CI}$ or a standard interview to mimic the effects of being sick while reporting. In both studies analyses

\footnotetext{
${ }^{1}$ There is no known systematic data to suggest what a "standard" contact tracing interview comprises. As discussed in the methods, the comparison contact tracing interview used in both studies was developed to represent a high-quality contact tracing interview, not necessarily whatever a "typical" contact tracing interview might be. To keep terminology consistent with past research, we refer to this comparison contact tracing interview as the "standard interview" throughout the dissertation.
} 
were conducted on the number of contacts provided (quantity), the average ease with which each contact could be found/identified based on the information provided (average precision of contacts) and the overall utility of the list of contacts (average precision * number of contacts).

\section{Hypotheses}

Study One. Because of the CI's robust support in the literature and strong basis in psychological theory we expect that the CI will yield more contacts than the standard interview. Specifically, we expect that the CI will generate all the same contacts that the standard interview would generate, with those contacts having at least the same level of precision as those that would be obtained via the standard interview. However the $\mathrm{CI}$ is expected to also elicit additional contacts beyond what would be elicited from the standard interview.

The most identifiable (i.e., precise) contacts (e.g., spouse, family member, roommate) are less likely to be omitted (and thus require less cuing) than less identifiable contacts (e.g., classmate, yoga instructor, adjacent passenger on plane). Thus, the extra contacts generated by the $\mathrm{CI}$ only are expected to have lower average precision than the contacts listed in the standard interview, because they required additional cuing to recall.

Thus, we hypothesize 1a) the CI will yield more contacts than the standard interview 1 b) the average precision of the contacts elicited by the CI will be less precise than those elicited by the standard interview, and 1c) the overall utility of the responses provided during the $\mathrm{CI}$ will be greater than those provided by the standard interview. 
Study Two. Regarding the different interview types the same hypotheses hold, 2a) the CI will yield more contacts than the standard interview $2 b$ ) the average precision of the contacts elicited by the CI will be less precise than those elicited by the standard interview, and $2 \mathrm{c}$ ) the overall utility of the responses provided during the CI will be greater than those provided by the standard interview.

Study Two introduces a new manipulation: the presence (or absence) of the cognitive impairment task. Because retrieval is an effortful task, we predict that recall will be negatively impacted by the presence of a distracting secondary task because of the reduction in resources available for retrieval. Specifically, we hypothesize when the task is present, versus absent, 2d) there will be fewer contacts reported, 2e) the average precision of the contacts will be lower and consequently $2 \mathrm{f}$ ) the overall utility of the responses will be lower. Note, it is also possible that average precision will in fact improve if, as a result of the limited resources available, only highly identifiable contacts are reported and less identifiable contacts are omitted, rather than described in less detail. Regarding potential interaction between the presence of the impairment task, and the interview type, there are two competing hypotheses. The first is that, consistent with past research, $2 \mathrm{~g}$ ) the CI will maintain its superiority over the standard interview in terms of quantity and utility, regardless of impairment (i.e., there will be no interaction). However, the past research is not directly on point, as it dealt with ID individuals, not individuals with a temporary limitation to their resources during retrieval only. Thus, the alternate hypothesis is that, $2 \mathrm{~h}$ ) because of the lack of resources to devote to the cognitively demanding CI, the CI will lose its advantage over the standard interview (i.e., there will 
be an interaction such that the CI is superior to the standard interview in the no impairment condition, but less beneficial in the impairment condition).

\section{STUDY ONE METHOD}

\section{Participants}

Fifty-two participants were recruited via SONA systems and in-class recruitment from a pool of undergraduate students enrolled at a large southeastern university. ${ }^{2}$ Course credit was provided for participation. Two participants were excluded because they were friends with the interviewer. Familiarity between the interviewer and participant was a concern because in at least one case the interviewer was listed as a contact of that participant. One participant was excluded because of experimenter error (non-random assignment to condition). The sample $(N=49)$ was primarily female $(86.3 \% ; 13.7 \%$ male) and Hispanic (76.5\%; 3.9\% White (not Hispanic or Latino); $17.6 \%$ African American; 2\% Asian/Pacific Islander). The mean age of participants was $22(S D=5)$ with a range of 18 to 50 .

\section{Interview Protocols}

Standard Interview Protocol. The standard contact tracing interview (see Appendix B) was developed to be representative of a high-quality contact tracing interview conducted by a skilled epidemiologist. For the purposes of both studies, specific contact information (e.g., phone number) for each contact was not required. Rather, the focus of the present studies was on the ability to remember more names,

\footnotetext{
2 Based on a power analysis using G*Power (Faul, Erdfelder, Lang, \& Buchner, 2007), a sample size of 40 participants was estimated to provide $80 \%$ power to detect group mean differences of large ( $\mathrm{f}=.4$ ) effect size (based on large effect sizes in previous research; e.g., Memon et al., 2010), using an ANOVA with a critical alpha of .05. We increased the target sample size by 5 per cell after the first days of testing indicated that there was a larger amount of variance in the contacts reported than expected.
} 
places, and details rather than the ability to provide contacts' phone numbers. The exact form used is listed in Appendix C. The interviewer filled in each slot on the form throughout the duration of the interview with the identified contact, descriptions of the contact, the location where the contact took place, and the type of contact (e.g., kiss, handshake). Prior to giving the standard procedural instructions (see the procedure section below), the interviewer developed rapport with the participant, which included a friendly exchange with the participant by asking, for example, whether the participant found parking easily at school that day. After the procedural instructions were provided, the interviewer asked about the participants" "circles of contacts" that should be listed if they had physical contact with the participant over the past three days. The circle of contacts began with the most intimate contacts (e.g., live with or significant other) and then broadened into work/school colleagues, friends, and general acquaintances (e.g., Publix cashier). After every contact provided, the interviewer followed-up immediately with questions about where the contact took place, a description of the person, and the type of contact that had occurred. Following the "circles of contacts", participants were shown a blank calendar and were asked with whom they interacted on each of the days (e.g., Monday, Tuesday, and Wednesday). Interviewers completed each section by asking if there were any additional contacts the participant could remember.

Cognitive Interview Protocol. The CI protocol was semi-structured and adhered to the following procedure (Appendix D). First, the interviewer established rapport by developing a connection between themselves and the participant (e.g., discussed the their common issues with traffic that morning, or how they are both from the same neighborhood). After rapport was developed, the interviewer instructed the witness to 
report exhaustively and established the social dynamics of the interview (see Appendix D for full description of interview procedure). To further demonstrate the need for a lengthy report, the interviewer provided a narration of an ideal interview, in which a very detailed description of an event and the people encountered was provided (e.g., his or her morning at work). The interviewer then asked for a first recall of everything the participant did and everyone with whom they had contact over the past three days (going through each day separately). During the first recall participants were presented with the blank calendar as a reference to the critical days. Throughout all free recalls, interviewers were instructed not to interrupt the participant and to save (and make a note of) any follow-up questions related to each contact for the end of the interview. After the first recall, participants went through a second free recall. During the second recall participants were asked to close their eyes (to aid in concentration) and instead of thinking about what they were doing on each day (as they did in the first recall), to think about all of the places they had been and all of the people who they encountered (varied retrieval). But instead of listing those places in chronological order, in this second recall participants were asked to list them in reverse order for each respective day. Participants were then asked to close their eyes again and were prompted to develop a rich mental image (context reinstatement) about one particular instance, which the interviewer deemed important to readdress (e.g., a time when a lot of people were present). Once participants felt as if a rich mental image was developed, they were prompted to provide a detailed narrative response about everything that happened (e.g., out to dinner with family). Participants then narrated while drawing a sketch of another scene in which many contacts were present (if possible a scene other than the one for which the context reinstatement was completed). The interview 
concluded with a request for participants to go through their "circle of contacts" and by filling out the same form used in the standard interview (Appendix C) by asking specific questions about each of the contacts listed in his or her notes (e.g., What is her last name? What type of contact did you have?). Throughout the CI the interviewer was encouraged to ask "anything else", or "anyone else" to probe for additional contacts.

Note, reporting a "circle of contacts" is not typically part of a CI. The CI is meant to improve upon a standard interview by using techniques appropriate for that particular witness or subject matter. In the real world, a specific practice used to gather critical information for that particular subject matter (as is the "circle of contacts" for contact tracing interviews) would be incorporated into the CI. In the present study, the circle of contacts was therefore included to ensure that all relevant contacts (e.g., people lived with) were listed.

\section{Interviewers}

Four undergraduate research assistants (RAs) conducted both the CI and the standard contact tracing interviews during the duration of data collection and switched between the protocols depending on which condition participants were randomly assigned to. In the interviewing literature there are competing schools of thought regarding whether the same interviewers should conduct all interviewing protocols (e.g., CI and standard interview), or whether one group of interviewers should conduct a particular condition (e.g., CI), and a different group of interviewers conduct the other condition (e.g., standard interview); each approach has advantages and disadvantages (e.g., Hershkowitz, Lamb \& Katz, 2014). In the present study we trained RAs to conduct both CIs and standard interviews to ensure that any differences found in interview 
outcomes were not driven by the specific characteristics of the interviewers selected to conduct the particular interviewing protocol, but rather were caused by the differences in the interview protocols themselves. One of the disadvantages of having RAs conduct both types of interviews is that there may be leakage from one interview protocol to another. For example, an interviewer may mistakenly ask follow-up questions in the middle of a CI, because he or she was trained to do so for the standard interview. Given the highly structured nature of the interviews in the present study, however, we were confident that little leakage would occur between interview protocols.

\section{Interviewer Training}

Standard Interview Training. A 2-hour training was provided for the standard interview. Because no known training is provided for standard contact tracing procedures, this training was provided to ensure that the interviewers were able to reliably replicate the prescribed standard procedure. During training, interviewers were instructed on the steps of the protocol, given handouts detailing the proper procedures and engaged in practice exercises. At the beginning of training, a cheat-sheet on the steps of the standard interview was provided for use both in training and also during the formal participant interviews (see Appendix E).

Cognitive Interview Training. The CI training was provided in a manner similar to that given for real-world practitioners and consisted of one, 5-hour session. In the first phase of training, interviewers were instructed on the general psychological principles underlying the $\mathrm{CI}$ and were also introduced to the Social Dynamics phase of the CI. In a second phase of training, interviewers learned the specific techniques used to enhance the witnesses' cognitive processes (context reinstatement, multiple retrieval, and eye 
closure). Interviewers were then trained on issues of communication (the sketch).

Practice exercises for each of the CI elements were completed throughout each training session. After each exercise, critical feedback was provided by the author.

At the beginning of training, the interviewers were given a cheat sheet outlining the CI protocol (see Appendix F). This sheet was used as a guide throughout practice exercises as well as during the formal interviews.

\section{Interviewer Quality Assurance}

After training was completed, each RA interviewed and recorded two pilot participants (one from each interview protocol) to assess adherence to training (as determined by the author). Feedback was subsequently provided and interviewers were instructed on any changes required to meet quality standards. One more practice interview was required and examined by the author in order to ensure that, if changes were required, they were applied. For those RAs for whom changes were not necessary after the first practice, a second interview was still required to ensure that the first successful interview was not successful merely by chance. As long as all protocols were adequately followed, the RA was allowed to begin the formal interview phase (all RAs met standards after the second round of practice).

\section{Disease}

The disease modeled in the present study was meningococcal meningitis. Meningococcal meningitis was selected because it a) would be familiar to college students, b) involved droplet transmission (transferred via large respiratory droplets; e.g., sneeze), like Ebola, but also included its spread through physical touch (increasing the amount of variability to detect statistical differences), and c) had an incubation period 
(usually about 3 days) conducive to practical methodological constraints (i.e., an hourlong interview). The 3-day incubation period ("Describe your contacts over the past three days.") allowed for enough time to list people from various activities (e.g., school, work, home), but not so much time that the interview would take more than one hour.

\section{Procedure}

Prior to participation, participants were assigned randomly to either a CI or a standard contact tracing interview. It was clear from initial testing that the day of the week in which the contacts were collected influenced the amount of contacts recalled. For example, if contacts were collected on a Monday, the participant typically listed fewer contacts than if contacts were collected on a Thursday. Thus, it was critical that any one type of interview did not fall exclusively on a day of the week where participants tend to recount fewer contacts. This was especially important since interviews were only collected over the course of a few weeks (restricting the ability for the random assignment to naturally balance out over time). Therefore, during random assignment, prior to data collection, participants were assigned such that one condition (SI or CI) did not comprise more than $60 \%$ of the interview types for a given day (e.g., out of 5 interview slots on Monday, only 3 could be taken up by a CI or a SI; out of 6 interview slots, only 3 could be taken up by a CI or SI). If the random number generator indicated that, for example, 4 out of 5 timeslots should be a standard interview (or CI), the author used the random number generator to decide which of those 4 timeslots would now be a CI (or standard interview) condition.

Upon arrival to the interview rooms, participants were consented and completed a standard demographic form. Participants were instructed they were participating in an 
interview to assess with whom they had interacted over the past three days (see Appendix $\mathrm{G}$ for full script of instructions provided to participants). The interviewer then stressed the importance of the contact tracing procedure (reminding them of the devastation of the recent Ebola outbreak), and asked them to imagine that they had been feeling ill the past 3 days. Participants were then instructed that they would be listing individuals with whom they had interacted over the past 3 days (including any type of physical interaction (e.g., hug or kiss) or anyone they may have shared saliva with (e.g., shared cigarette or drink). Finally, the participants were told that they would need to provide details about the interaction, including information about the person (first name, last name, and description), the location of the interaction, and the specific type of contact. After these instructions were provided, the interviewer began the assigned protocol. The interviewers were given a total of one hour to complete their procedure.

\section{Scoring}

All interviews were audio recorded by a digital MP3 recorder and transcribed by a professional transcribing service $(n=47)$ or undergraduate research assistant $(n=2){ }^{3}$

The dependent variables of interest are the number of contacts provided (quantity), how easy it would be to find the reported contacts (average precision), and a score of the overall utility of the contacts provided (average precision * number of contact provided). In order to score for both of these measures, undergraduate research assistants $(N=6)$ scoured transcripts for every contact listed. These contacts were then transferred into a spreadsheet, which closely resembled the sheet completed by interviewers during the

\footnotetext{
${ }^{3}$ Funds were only able to cover the cost of 47 interviews, leaving 2 to be transcribed by an undergraduate.
} 
interviews (Appendix H). In addition to creating an exhaustive list of all of the contacts, additional information not requested by the original contact tracing sheet was included in the spreadsheet (i.e., the contact's relationship to participant, and whether the contact lives with the participant). The corresponding list created by the RAs was used in lieu of the interviewers' notes during scoring in order to ensure that the most exhaustive version of the contact list was used (because the interviewer may have missed some details).

Prior to being used for scoring, each RA-generated spreadsheet was crosschecked against the original interviewer's contact sheet notes. If there was a disagreement between the two (e.g., the spreadsheet was missing a contact who was listed in the notes), the transcript was referred to and whatever was present in the transcript was provided in the final version of the spreadsheet. Differences in the spreadsheet and the interviewers notes were rare and typically comprised one contact included in the spreadsheet but not in the interviewer's notes.

Scoring for Quantity. Each person listed in the spreadsheet was scored by the author, who was the primary scorer and blind to condition, to determine whether the individual would be considered a contact by an epidemiologist (see Appendix I for the quantity scoring protocol). First, the total number of contacts listed was noted (regardless of type of contact). Because the present study's disease of interest, meningococcal meningitis, is spread through droplet transmission, this was the primary contact-type of interest (participants were asked for people with whom they had physical contact with or shared saliva). Thus each of the "total contacts" identified was subsequently categorized as either a contact for a droplet-transmitted disease specifically, or not a contact for a droplet-transmitted disease. To score for whether the contact would be droplet- 
transmitted contact and thus at high-risk for meningococcal meningitis, the type of interaction (e.g., hug, kiss, shared a drink or utensil) and/or whether the contact and participant lived together, was considered (see Appendix $\mathrm{J}$ for a source on who would be considered a high-risk contact for meningococcal meningitis; Pickering, Baker, \& Kimberlin, 2012). Once participants were classified as being a droplet-transmitted contact (or not a droplet-transmitted contact), the number of droplet-transmitted contacts were counted.

Scoring for Precision and List Utility. Each contact and the details associated with that contact were also scored by the author, who was the primary scorer and blind to condition, for precision (see Appendix K for the precision scoring protocol). Precision was conceptualized as a measure of how likely it would be to find the reported contact. Precision was scored on the following 0 to 3 scale: 0 being impossible to find (no name or description; e.g., kids running around in a park), 1 being difficult to find (no name, but helpful description; e.g., waiter at Cheesecake Factory who was tall, and blonde), 2 being likely to find (first name and a description; e.g., John in my psych class at FIU who is Cuban and has black hair and brown eyes), and 3 being easy to find (first and last name; John Smith, my friend from class at FIU). An average precision score (total precision score divided by total number of contacts) was calculated in order to gauge the ease of finding the contacts.

We were also interested in the overall usefulness of responses to the interview; both precision and quantity play into the whether the interview should be considered effective. The utility score was calculated by multiplying the average precision score for a participant by the number of contacts that participant provided (note, this is the same as 
the sum of the precision scores across all contacts). The average precision scores and the utility scores were calculated for a) the total contacts listed and b) the droplet-transmitted contacts specifically.

\section{Reliability Scoring}

An undergraduate research assistant co-scored 15\% $(n=8)$ of the spreadsheets already scored by the author for quantity and precision. Reliability was calculated using Kappas and was .94 for scoring for droplet-transmitted quantity and .84 for scoring for precision.

\section{STUDY ONE RESULTS}

A series of t-tests was conducted to examine the effects of interview type on the total number of contacts reported and the number of droplet-transmitted contacts reported (testing hypothesis 1a). For each of these types of contacts, separate t-tests were also conducted to test whether an average measure of precision and the measure of overall utility varied as a function of interview type (testing hypotheses $1 \mathrm{~b}$ and $1 \mathrm{c}$ ). Two outliers, defined as scores more than 2 standard deviations from the mean on total contacts listed, were identified and excluded from analyses. As suggested by McClelland (2000) any differences in conclusions that would be made if outliers were included in the analyses will be reported. Only one analysis differed when outliers were included and is reported in the "Overall Utility" section below.

\section{Quantity of Contacts}

The CI generated significantly more total contacts $(M=16.91, S D=6.90,95 \%$ $\mathrm{CI}[13.93,19.90])$ than the standard interview $(M=11.88, S D=6.02,95 \% \mathrm{CI}[9.33$, $14.42]), t(45)=2.67, p=.011, d=.78$. There was, however, no significant difference in 
the number of droplet-transmitted contacts generated by the CI $(M=10.96, S D=6.09$, $95 \% \mathrm{CI}[8.32,13.59])$ compared to the standard interview $(M=9.50, S D=6.60,95 \%$ $\mathrm{CI}[6.71,12.29]), t(45)=.79, p=.437, d=.23$. Thus, our hypothesis (1a) that the CI would increase the number of contacts listed compared to the standard interview, was supported for the total contacts listed, but not for the droplet-transmitted contacts listed.

\section{Average Precision of Contacts}

Precision was measured on a scale of 0 to 3, with 0 being not likely to find the contact, and 3 being extremely likely to find the contact. ${ }^{4}$ Higher average scores indicate a higher level of precision. Results suggested no significant difference in the average precision of the total contacts listed for the CI $(M=2.17, S D=.41,95 \% \mathrm{CI}[1.99,2.35])$ compared to the standard interview $(M=2.33, S D=.45,95 \% \mathrm{CI}[2.14,2.52]), t(45)=$ $1.29, p=.205, d=.38$. Although the difference was not significant, the effect size was moderate. There was also no difference in the average precision of droplet-transmitted contacts listed for the $\mathrm{CI}(M=2.61, S D=.34,95 \% \mathrm{CI}[2.46,2.76])$ compared to the standard interview $(M=2.66, S D=.33,95 \% \mathrm{CI}[2.51,2.8]), t(44)=.45, p=.656, d=.13$. These findings fail to support our hypothesis (1b) that the average precision of responses would be higher in the standard interview than in the CI.

\section{Utility of Contacts}

There were no significant differences in the utility of the responses provided for the total contacts listed in the CI $(M=35.27, S D=14.73,95 \% \mathrm{CI}[28.85,51.59])$ compared to the standard interview $(M=28.21, S D=16.96,95 \% \mathrm{CI}[21.05,35.37]) t(45)$

\footnotetext{
4 Although the outcome variables average precision and total utility are bounded at 0 and 3 and are only coarsely continuous, violations of normality are minor. Predicted means for each condition are within the bounds of the observed variables; skew within each condition is low, ranging from .04 to 1.75. Thus, standard normal models were used.
} 
$=1.51, p=.138, d=.44$. Although the effect did not reach significance, the effect size was moderate. Note that when outliers were included in the analyses, the overall utility of responses for the total contacts listed was significantly higher in the CI compared to the standard interview ( $p=.041, d=.60)$. There was also no difference between the CI ( $M=$ $27.61, S D=14.63,95 \% \mathrm{CI}[21.28,33.94])$ and standard interview $(M=26.09, S D=17.25$, $95 \% \mathrm{CI}[18.63,33.55])$ in the overall utility for the droplet-transmitted contacts provided $t(44)=.32, p=.748, d=.10$. Our hypothesis (1c) that the overall utility would be greater in the CI than the standard interview was therefore unsupported.

\section{Interview Length}

The length of each interview was measured in minutes. The CI $(M=33.55, S D=$ 14.24) lasted significantly longer than the standard interview $(M=13.7, S D=10.56)$ $t(45)=5.45, p<.001, d=1.58$. Interview length was significantly correlated with both total contacts $r(47)=.57, p<.001$ and droplet-transmitted contacts $r(47)=.35, p=.015$.

\section{STUDY ONE DISCUSSION}

The results clearly indicated a substantial increase in the number of total contacts reported compared to the standard contact tracing interview. Almost 35\% more contacts were provided by the CI compared to the standard interview (approximately 5 more contacts). When examining the droplet-transmitted contacts only, the CI produced a statistically non-significant $14 \%$ increase in droplet-transmitted contacts listed compared to the standard interview (approximately 1.5 more contacts). Although statistically nonsignificant, one could argue that in this context a single additional contact is important.

There were no significant differences between the CI and standard interview in the precision of the contacts listed. It was hypothesized that the standard interview would 
have a higher level of precision than the CI. This prediction was based on the expectation that the CI would generate all the same contacts that the standard interview would generate, and at the same level of precision, but also yield additional contacts at a lower level of precision than the standard interview (driving down the average precision score). Since, the CI generated more total contacts compared to the standard interview, there was a non-significant trend, whereby the precision score for total contacts was higher in the standard interview than the CI. However, because few additional droplet-transmited contacts were provided by the CI, there was no detectable difference in average precision between the $\mathrm{CI}$ and the standard interview.

There was also no significant difference between the CI and standard interview in the overall utility of the responses. Although statistically non-significant, the effect was of moderate size with the overall utility of the responses in the CI higher than in the standard interview. This suggests that there is likely a practical difference between the overall utility of the responses for the CI compared to the standard interview. Overall utility is tied closely to the number of contacts reported. Because the CI generated significantly more total contacts (but not droplet-transmitted contacts), with similar levels of precision, the difference in utility scores was moderate, but only for total contacts.

One might interpret the results regarding total contacts as suggesting that for a disease that is transmitted through the air (e.g., measles), the CI would be superior to the standard interview, as "total contacts" would be relevant in that situation. However, any findings reported on the total contacts listed should be evaluated cautiously. While in both the $\mathrm{CI}$ and standard interview, participants were given the same initial instructions regarding the task (i.e., the interviewer needs to know about contacts you had physical 
contact with), during the CI participants were generally encouraged to narrate freely about all of the people they encountered, regardless of whether physical contact was made (to avoid stunting recall by restricting it to only physical contacts). In the standard interview, however, participants were asked only to report people with whom they had a physical exchange, shared saliva, or lived with. To be able to make a valid comparison between the CI and standard interview for total contacts (i.e., contacts that are not droplet-transmitted contacts, but potentially relevant contacts for an airborne illness), the standard interview should also ask for the non-physical contacts as well. We are therefore wary to conclude from the findings on total quantity that the CI significantly increases the amount of contacts listed relative to the standard interview for a disease in which nonphysical contacts would also be of interest. Nevertheless, these findings are encouraging. Much of the CI's value lies in its ability to generate information not directly requested by the interviewers. As such, total quantity findings signify that the CI was generating more information than the standard interview, even if the increase was not in the target information per se.

\section{Practical Implications}

Study One was a first attempt at testing whether the well-established CI increased the number of contacts reported compared to a typical contact tracing interview (i.e., the standard interview). In the present study, the CI generated approximately 1.5 more droplet-transmitted contacts than the standard interview. Although statistically nonsignificant, in a practical sense, generating even one more contact may be critically important. For instance, in some diseases contacts have a high probability of becoming dangerously ill and infecting others. When this is the case, the identification of an 
additional contact may mean the difference between whether or not many people receive treatment, infect others, or succumb to the disease.

\section{Limitations}

In Study One, we appeared to have a lack of statistical power. Future research would benefit from examining the differences between the CI and standard interview with a larger sample size. The power analysis that suggested the sample size used here would be sufficient was based on the large effect sizes reported in much of the CI literature. However, it seems that in a contact tracing context, particularly when only droplet-transmitted contacts are considered, the effect size is relatively smaller. Thus, power was lower than intended. A second limitation is that the individuals interviewed in the present study were presumably healthy. Reporting during contact tracing often occurs when the patient is acutely ill. Since research suggests that acute infection impairs cognitive functioning, it is critical to evaluate how cognitive impairment affects reporting during a contact tracing interview.

In light of the identified limitations, in Study Two we a) increased the sample size per cell, thereby increasing power to detect differences and b) introduced a cognitive impairment task to model being interviewed while ill.

\section{STUDY TWO METHOD}

\section{Participants}

A total of 157 participants were recruited via SONA systems and in-class recruitment from a pool of undergraduate students enrolled at a large southeastern 
university. ${ }^{5}$ Course credit was provided for participation. Nine participants were excluded from analyses because of experimenter error (started the impairment task at the wrong point in the interview), and two participants were excluded because of participant error (used cell phone to look up contacts $(n=1)$ or indicated unwillingness to report all contacts $(n=1))$. The final sample $(N=146)$ was primarily female $(75.0 \% ; 25.0 \%$ male $)$ and Hispanic (61.8\%; 9.7\% White (not Hispanic or Latino); $18.8 \%$ African American; 4.9\% Asian/Pacific Islander; 4.9\% Other). The mean age of participants was $21(\mathrm{SD}=4)$ with a range of 18 to 48 .

\section{Design}

A 2 (impairment task v. no impairment task) x 2 (CI v. standard interview) between subjects factorial design was used.

\section{Interviewers and Interview Protocols}

The same interviewers and interviewing protocols used in Study One were used in Study Two.

\section{Working Memory Impairment}

To select an appropriate working memory impairment task, there were important considerations. First, the impairment task could not itself directly interfere with the completion of an interview protocol. Relatedly, the interview procedure could not interfere with the completion of the task. Notably, in both interviews the witnesses must listen and respond to interview questions. As such, an auditory (e.g., press a button every

\footnotetext{
${ }^{5}$ Based on a power analysis using $G^{*}$ Power (Faul, Erdfelder, Lang, \& Buchner, 2007), a sample size of 140 participants was estimated to provide $80 \%$ power to detect group mean differences, of medium size (f $=.25$ ), using an ANOVA with a critical alpha of .05 .
} 
time you hear a beep) or oral task (e.g., count numbers backward by 3 starting with 100) could not be used. Similarly, during a CI participants draw a sketch. Thus, any task that might impede the ability to draw a sketch (and if drawing a sketch, would impede the completion of the impairment task) could not be used (e.g., sorting a shapes using hands only). Finally, because participants close their eyes during a CI, a visual task (e.g., indicate which color is being displayed on a screen every five seconds) could not be used.

Given these restrictions the task selected to interfere with working memory was a time-estimation procedure (referred to as "the impairment task" throughout the rest of the manuscript). During the task participants hit the spacebar on a keyboard with their nondominant hand every time they thought 7 seconds had passed. Notably, the task interfered with working memory, while also allowing participants to listen to the questions asked, close their eyes as instructed, orally respond to questions, and use their dominant hand to sketch. Time-estimation has been used as a secondary task in several studies on the mental workload associated with driving automobiles, piloting planes, conducting surgery, and other basic cognitive processes (Baldauf, Burgard, \& Wittmann, 2009; Grant, Carswell, Lio, \& Seales, 2013; Grant, Carswell, Lio, Seales, \& Clark, 2009; Liu \& Wickens, 1994).

A time-estimation website was used (http://stopwatch.online-timers.com/ stopwatch-with-time-intervals) on a standard laptop computer, with an external keyboard attached. During both a practice session and the formal testing the screen displaying the website and the running clock was turned away from the participant. The amount of time to be estimated was pilot tested and 7 seconds seemed to be most difficult (compared to 5 
seconds or 10 seconds). ${ }^{6}$ Participants were given a 5-minute practice session prior to the rapport section of the interviews. Participants were instructed that during the practice task their performance would be tracked and that they would have to continue practicing (in addition to the standard five minutes) if they did not perform well enough. For 30 seconds prior to the practice task, the participant completed the task while facing the countdown clock on the website (to provide a sense of how long 7 seconds was). After the 30 seconds was completed, the interviewer turned the screen back around, left the room, and let the participant practice the task for 5 minutes. The practice session served two purposes. First, it familiarized participants with how the procedure would be performed. Second, it imitated a sick patient coming in for an interview and having undergone cognitive impairment from the illness for some time before the interview had begun. Prior to completing the impairment task during the interview, participants were instructed that it was important to perform well on the task throughout the interview and that performance on the task would be measured. It was assumed that the task instruction would entice participants to take the task seriously. Pilot testing suggested that participants were able to complete the distraction task at a relatively high level of accuracy (hit the space bar around 7 seconds), and that it successfully made the interview portion more difficult for participants to complete.

At the end of the interview (and the completion of the task), the website provided an output of each participant's scores including the number of times the participant had

\footnotetext{
6 Interviewers pilot tested approximately 2 participants each, for a total of 8 participants to test how difficult it was to complete the time estimation task during an interview. Informal results suggested that the time estimation task caused reporting to be more difficult than when no task was used and that estimating that 7 seconds had passed was more difficult than estimating that 5 or 10 seconds had passed.
} 
pressed the spacebar, and the time that passed after each hit of the spacebar (measured performance on the task).

\section{Debriefing Questionnaire}

Participants completed a debriefing questionnaire evaluating their subjective perceptions of the interview and task. The questionnaire asked participants to rate on a 7point scale the ease or difficulty of remembering their contacts, the mental effort expended during the interview, and how successfully they remembered their contacts (see Appendix L). Participants in the cognitive impairment conditions were additionally asked to rate how difficult it was to complete the task during the interview, and to estimate what percentage of their mental attention (divided as they please between 100\%) was relegated to the task versus responding to the interview (see Appendix M). Note that, because of a clerical error, the anchor for the question about difficulty completing the time estimation task (i.e., how difficult was it to complete the time estimation task during the interview) was erroneous. Instead of anchoring for difficulty, it asked participants to rank the subjective effectiveness at completing the task ( 1 being not at all effectively and 7 being extremely effectively). Since the anchors did not match the question asked, conclusions surrounding the question on difficulty should be evaluated cautiously.

\section{Disease}

The disease (meningococcal meningitis) and number of days of contacts to list (3

days) was the same as in Study One. It is important to note that although we are modeling the types of contacts of interest as well as the incubation period of an outbreak of meningococcal meningitis, the cognitive impairment imposed via the impairment task is not meant to be representative of the impairment associated with meningococcal 
meningitis. In fact, the cognitive impairment associated with meningococcal meningitis can be as extreme as a being unable to form cogent sentences, or being in a coma. As such, the cognitive impairment imposed in the present study was simply meant to serve as a proxy for the potential impairment associated with various types of acute infection, not necessarily meninicoccal meningitis.

\section{Procedure}

Participants were assigned randomly to one of the four conditions prior to participation. As in Study One, the author ensured that no one out of the four conditions was assigned more than $60 \%$ of the time for each day. Upon entering the lab, all participants were consented, and completed a demographic form. For participants in conditions that involved no impairment task, the rest of the procedure followed that of Study One (complete either a CI or standard contact tracing interview). Participants in the cognitive impairment conditions were instructed that they must complete an additional task while completing their interview and that they must first complete the task alone for five minutes as practice. After the five-minute practice round, both types of interviews commenced as described in Study One (i.e., the interviewer went over the contact tracing instructions and conducted either a CI or a standard contact tracing interview). See Appendix $\mathrm{N}$ for an experimental protocol for a condition with an impairment task. After the interview participants in all condtions completed the debriefing questionnaire.

\section{Scoring}

All interviews were audio recorded by a digital MP3 recorder and transcribed by undergraduate research assistants. As in Study One the dependent variables of interest are 
quantity, precision, and utility, and all responses were transferred into a spreadsheet by research assistants for scoring.

Scoring for Quantity, Precision, Utility and Inter-Rater Reliability. Quantity and precision were scored in the same fashion and by the same scorers (author as primary scorer) as in Study One. Fifteen percent of the interviews $(n=22)$ were co-scored by an undergraduate RA. Kappas were .96 for scoring for droplet-transmitted quantity and .85 for scoring for precision.

\section{STUDY TWO RESULTS}

A series of 2 (interview type) x 2 (presence of the impairment task) between subjects ANOVAs were conducted to test the effects of interview type and presence of the impairment task on measures of quantity (testing hypotheses $2 \mathrm{a}, 2 \mathrm{~d}, 2 \mathrm{~g}$ and $2 \mathrm{~h}$ ), average precision (testing hypotheses $2 \mathrm{~b}$ and $2 \mathrm{e}$ ) and overall utility (testing hypotheses $2 \mathrm{c}$ and 2f) for both total contacts and droplet-transmitted contacts. In addition, a one-way (interview type) between subjects ANCOVA was conducted to test whether performance on the distraction task was associated with the number of contacts reported. A 2 (interview type) x 2 (presence of the impairment task) between subjects MANOVA was used to test the effects of interview type and presence of impairment on three of the debriefing questions involving subjective assessment of the interview experience, and a one-way (interview type) MANOVA was used to test the effects of interview type on two subjective questions about completing the distraction task. Finally, a 2 (interview type) $\mathrm{x}$ 2 (presence of the impairment task) between subjects ANOVA was used to examine the effects of interview type and presence of the impairment task on length of the interview. Eight outliers, defined as scores more than 2 standard deviations from the mean on total 
contacts listed, were identified and excluded from the analyses. As suggested by Mclleland (2000), if results differ when outliers are present, they will be reported. There were several differences in conclusions when outliers were included, and are reported in the "Quantity of Contacts", "Utility of Contacts" and "Interview Length" sections below. The discrepancy in the results when outliers are present seems to be largely driven by one particular outlier in the CI with impairment task condition. The outlier provided almost $80 \%$ more contacts than the average participant and $25 \%$ more than the next highest outlier; the outlier reported attending a large event at which she greeted a large number of people. When only this particular outlier is removed, the vast majority of analyses are the same with and without outliers present.

\section{Quantity of Contacts}

Total Contacts Quantity. A 2 x 2 ANOVA revealed a significant main effect of interview type, $F(3,134)=13.45, p=<.001, d=.63$, but not of presence of the impairment task on the total number of contacts reported, $F(3,134)=.60, p=.441, d=$ .13 (see Table 1 for all means, standard deviations and confidence intervals for total contacts). Results demonstrated that the $\mathrm{CI}(M=16.78, S D=7.55)$ led to significantly more contacts reported than the standard interview $(M=12.57, S D=6.02)$. However, the main effect of interview type was qualified by a significant interaction effect, $F(3,134)=$ $5.17, p=.025, d=.41$ (see Figure 1). Follow-up analyses suggested that when there was no impairment task completed, the $\mathrm{CI}(M=18.52, S D=7.50)$ generated significantly more contacts than the standard interview $(M=11.71, S D=5.91), t(64)=4.12, p<.001$, $d=1.01$. However, when the impairment task was completed, there was no difference in the number of contacts generated by the $\mathrm{CI}(M=15.03, S D=7.32)$ compared to the 
standard interview $(M=13.43, S D=6.10), t(70)=1.01, p=.317, d=.24$. Note that when outliers were included in analyses, the interaction was not significant $(p=.204, d=$ .21). The interaction effects for total quantity seem to support our hypothesis $(2 \mathrm{~h})$ that because of a lack of resources to devote to the CI, the CI would be superior to the standard interview only when no impairment task was used, and not the competing hypothesis $(2 \mathrm{~g})$ that the CI would increase the number of contacts regardless of whether or not the impairment task was completed.

As previously noted, results surrounding total contacts should be interpreted with caution. Only the CI conditions (with and without task) encouraged participants to generate all of their encountered contacts, and not just physical contacts. As such, we tested whether the impairment task affected the total number of contacts reported for just the CI conditions (in which less emphasis was placed on droplet-transmitted contacts). To do this, the interaction was re-assessed to determine whether completing the impairment task significantly decreased the number of contacts reported within each interview type. Results suggested that the impairment task reduced the number of contacts reported in a CI (compared to when no impairment task was present), but only with marginal significance $t(64)=1.91, p=.061, d=.47$. However, the impairment task did not significantly affect the number of contacts reported in a standard interview (compared to when no impairment task was present), $t(70)=1.21, p=.229, d=.29$.

Droplet-Transmitted Quantity. A 2 x 2 ANOVA revealed no significant main effect of interview type on the number of droplet-transmitted contacts $F(3,134)=2.13, p$ $=.147, d=.26$ (see Table 2 for all means, standard deviations, and confidence intervals for droplet-transmitted contacts). Note that when outliers were included in analyses, there 
was a significant main effect of interview type $(p=.010, d=.43)$, such that the CI generated significantly more droplet-transmitted contacts compared to the standard interview. There was also no significant main effect of presence of the impairment task $F(3,134)=.17, p=.683, d=.06$. There was, however, a significant interaction effect, $F(3,134)=7.09, p=.009, d=.46$ (see Figure 2$)$. Follow-up analyses indicated that when no impairment task was completed, the CI yielded significantly more contacts $(M=$ 14.07, $S D=6.60)$ than the standard interview $(M=9.69, S D=5.42), t(64)=2.96, p=$ $.004, d=.87$. However, when the impairment task was completed, there was no significant difference between the CI $(M=10.8, S D=6.52)$ and standard interview $(M=$ $12.08, S D=6.34)$ in number of contacts reported, $t(70)=.85, p=.401, d=.20$. Note that when outliers were included in analyses, the interaction was only marginally significant $(p=.066, d=.31)$. The significant interaction found for droplet-transmitted contacts supports our hypothesis (2h) that the CI would generate more contacts than the standard interview, but only when no impairment task was used.

\section{Average Precision of Contacts}

Total Contacts Average Precision. Precision was measured on a scale of 0 to 3 , with 0 being not likely to find the contact, and 3 being extremely likely to find the contact (higher scores indicate higher levels of precision) ${ }^{7}$. A 2 × 2 ANOVA demonstrated a significant main effect of interview type on the average precision of total contacts reported $F(3,134)=5.92, p<.001, d=1.09$ (see Table 1 for all means, standard deviations and confidence intervals). Results suggested that the standard interview $(M=$

\footnotetext{
7 Although the outcome variables average precision and total utility are bounded at 0 and 3 and are only coarsely continuous, violations of normality are minor. Predicted means for each condition are within the bounds of the observed variables; skew within each condition is low, ranging from .11 to 2.10. Thus, standard normal models were used.
} 
2.50, $S D=.29)$ generated a significantly higher average precision score than the $\mathrm{CI}(M=$ $2.08, S D=.47)$. There was no significant main effect of impairment task, $F(3,134)=$ $.002, p=.963, d=.00$, or a significant interaction effect, $F(3,134)=.41, p=.524, d=$ .12. The significant main effect of interview type supports our hypothesis $(2 b)$ that the average precision of contacts listed would be higher in the standard interview compared to the $\mathrm{CI}$.

Droplet-Transmitted Average Precision. A 2 x 2 ANOVA demonstrated a significant main effect of interview type on the average precision of droplet-transmitted contacts, $F(3,134)=21.43, p<.001, d=.81$ (see Table 2 for means, standard deviations and confidence intervals). Results suggested that the standard interview $(M=2.67, S D=$ .27) generated significantly higher average precision scores than the CI $(M=2.37, S D=$ .46). There was no significant main effect of impairment task $F(3,134)=.08, p=.450, d$ $=.13$, or significant interaction effect, $F(3,134)=.89, p=.348, d=.17$. Once again, these results support our hypothesis (2b) that the average precision of contacts listed would be higher in the standard interview compared to the CI.

\section{Utility of Contacts}

Total Contacts Utility. A 2 x 2 ANOVA revealed no significant main effects of interview type, $F(3,134)=2.36, p=.127, d=.26$, or presence of the impairment task on the overall utility of total contacts reported, $F(3,134)=.17, p=.684, d=.06$ (see Table 1 for means, standard deviations and confidence intervals). Note that when outliers were included in analyses, there was a significant main effect of interview type $(p=.011, d=$ .43), such that the overall utility of the CI was higher than the utility of the standard interview. There was, however, a significant interaction effect, $F(3,134)=4.15, p=.044$, 
$d=.35$ (see Figure 3). Follow-up analyses suggested that when no impairment task was completed, the overall utility of the $\mathrm{CI}(M=38.81, S D=18.64)$ was higher than the utility of the standard interview $(M=28.69, S D=13.47), t(64)=2.55, p=.016, d=.62$, but when the impairment task was completed, there was no difference in the utility of the $\mathrm{CI}(M=31.89, S D=18.50)$ compared to the standard interview $(M=33.30, S D=15.58)$, $t(70)=.35, p=.726, d=.08$. When outliers were included in analyses, the interaction was not significant $(p=.282, d=.18)$. We had hypothesized that overall utility would be higher in the CI than the standard interview (2c) and would be reduced by the presence of the impairment task (2f). The interaction partially supports hypothesis $2 \mathrm{c}$, and supports hypothesis $2 \mathrm{f}$. Results demonstrated that utility was higher in the CI (supporting the hypothesized superiority of the CI), but only when the impairment task was not completed (supporting the hypothesized reduction as a function of impairment).

Droplet-Transmitted Utility. A 2 x 2 ANOVA demonstrated no significant main effects of interview type, $F(3,134)=.176, p=.675, d=.06$ or presence of the impairment task on overall utility for droplet-transmitted contacts, $F(3,134)=.214, p=$ $.645, d=.09$ (see Table 2 for means, standard deviations and confidence intervals). There was, however, a significant interaction between interview type and presence of the impairment task $F(3,134)=6.57, p=.011, d=.44$ (see Figure 4). Follow-up analyses suggested that when no impairment task was completed, the overall utility of the CI was higher $(M=33.55, S D=16.34)$ than in the standard interview $(M=25.57, S D=14.99)$, $t(64)=2.18, p=.033, d=.53$. However, when the impairment task was completed, there was no significant difference in the overall utility of the CI $(M=25.46, S D=16.75)$ compared to the standard interview $(M=31.19, S D=15.67), t(70)=1.48, p=1.43, d=$ 
.35. When outliers were included in analyses, the interaction was not significant ( $p=$ $.107, d=.27)$. As with results surrounding the utility of total contacts, the interaction effects for droplet-transmitted contacts provide support for hypotheses $2 \mathrm{c}$ and $2 \mathrm{f}$.

\section{Task Performance}

Participants' performance on the distraction task was calculated as the average distance of each hit of the spacebar from 7 seconds (i.e., average error from the desired score of 7 seconds). One potential concern with the distraction task was that there would be differential performance across the $\mathrm{CI}$ and standard interview conditions (e.g., participants in the CI condition may not pay attention to the impairment task and thus perform worse on the task but better in the interview than participants in the standard condition). In order to test whether performance on the distraction task explained any variance, a one-way ANCOVA was performed using droplet-transmitted contacts as the dependent variable, interview type as the factor and average error score on the distraction task as the covariate. Controlling for performance on the impairment task, there was still no significant difference between the CI with the impairment task $(M=10.65, S D=6.52$, $95 \%$ CI $[8.49,12.82])$ and the standard interview with the impairment task $(M=12.01$, $S D=6.28,95 \% \mathrm{CI}[9.87,14.14]), F(1,68)=.783, p=.379, d=.20$ and the covariate (error score) did not have a significant effect, $F(1,68)=1.50, p=.225, d=.30$. An additional t-test with the error score as the dependent variable also demonstrated that there was no significant difference in performance on the distraction task in the CI condition $(M=3.26, S D=1.58,95 \% \mathrm{CI}[2.69,3.80])$ and the standard interview condition $(M=2.74, S D=1.71,95 \% \mathrm{CI}[2.19,3.30]), t(69)=1.32, p=.190, d=.32$. 


\section{Debriefing Questionnaire}

Subjective Assessments of Interview Experience. A 2 x 2 MANOVA was conducted with ratings of difficulty of remembering ( 1 being extremely easy and 7 being extremely difficult), mental resources expended (1 being no mental effort and 7 being extreme mental effort), and success of recall ( 1 being not at all successfully and 7 being extremely successfully) as the dependent variables and type of interview and presence of the impairment task as the factors. There was no significant multivariate effect of interview type, Wilks' Lambda $=.99, F(3,131)=.443, p=.723, d=.20$, but there was a significant main effect of presence of the impairment task, Wilks' Lambda $=.84, F(3$, $131)=8.35, p<.001, d=.87$. (See Table 3 for all means, standard deviations, and confidence intervals on debriefing questions.) An examination of the univariate effects revealed that presence of the impairment task significantly affected all three ratings, with the impairment task causing participants to find it significantly more difficult to remember contacts $(M=4.23, S D=1.49)$ compared to those without the task, $(M=3.51$, $S D=1.45), F(1,133)=7.88, p=.006, d=.51$, use significantly more mental resources during the interview $(M=5.39, S D=1.27)$ compared to those without the task $(M=4.51$, $S D=1.09), F(1,133)=17.85, p<.001, d=.74$, and perceive recall as significantly less successful $(M=4.94, S D=1.21)$ compared to those without the task $(M=5.45, S D=$ 1.26), $F(1,133)=6.04, p=.015, d=.41$.

The multivariate main effect of impairment task was qualified by a significant interaction with interview type, Wilks' Lambda $=.94, F(3,134)=3.05, p=.031, d=.52$. There were significant univariate effects for the interaction on difficulty remembering $F(1,134)=4.83, p=.030, d=.38$, and success of recall $F(1,134)=6.10, p=.015, d=$ 
.43 , but not for mental effort $F(1,134)=.726, p=.396, d=.14$. Follow-up analyses suggested that when no impairment task was used, the ratings by those in the standard interview compared to the $\mathrm{CI}$ did not differ on questions of difficulty remembering contacts, $t(63)=.98, p=.329, d=.25$ or success of recall $t(63)=1.45, p=.152, d=.36$. However, when the impairment task was used, the standard interview led to significantly higher ratings of difficulty remembering contacts compared to the $\mathrm{CI}, t(70)=2.16, p=$ $.035, d=.51$, and led to significantly lower ratings of success at remembering contacts compared to the $\mathrm{CI}, t(70)=2.07, p=.042, d=.49$ (see Figures 5 and 6 ).

Subjective Assessments of Task Experience. A one-way MANOVA was conducted with ratings of difficulty of completing the time estimation task ( 1 being not at all effectively and extremely effectively ${ }^{8}$, and amount of mental effort devoted to the task compared to the interview (out of $100 \%$ ) as the dependent variables, and interview type as the factor (only participants who completed the impairment task conditions completed this measure). There was no significant effect of interview type on ratings of the difficulty of the time estimation task or the percentage of mental effort devoted to the task, Wilks' Lambda $=.98, F(2,68)=80, p=.454, d=.31$ (see Table 4 for all means, standard deviations, and confidence intervals for questions about completing the task).

\section{Interview Length}

A $2 \times 2$ ANOVA demonstrated a main effect of interview type on length of the interviews (measured in minutes), $F(1,134)=201.27, p<.001, d=2.45$ (see Table 5 for all means, standard deviations and confidence intervals of length of interview). Results

\footnotetext{
${ }^{8}$ As a reminder, the anchors used for ratings of difficulty were included in error, and should have been 1 being extremely easy and 7 being extremely difficult. As such, results surrounding these findings should be evaluated with caution.
} 
suggested that the CI $(M=40.49, S D=11.44)$ lasted significantly longer than the standard interview $(M=19.58, S D=6.44)$. There was also a significant effect of the presence of the impairment task on the length of the interview, $F(1,134)=6.02, p=.015$, $d=.41$. Specifically, interviews lasted longer when the task was completed $(M=31.84$, $S D=11.06)$ compared to when no task was completed $(M=28.23, S D=16.16)$. Note that the main effect of impairment task is non-significant when outliers are present $(p=$ $.099, d=.28)$. The main effects were qualified by a significant interaction effect $F(1$, $134)=12.60, p=.001, d=.63$ (see Figure 7). Follow-up analyses revealed that the impairment task led standard interviews to be significantly longer in conditions with the impairment task $t(71)=6.82, p<.001, d=1.89$, however, the impairment task had no significant effect on the length of the CIs, $t(66)=.79, p=.434, d=.14$.

\section{STUDY TWO DISCUSSION}

Results plainly demonstrated an advantage of the CI over the standard interview in generating more total contacts (approximately 7 more contacts) and droplet-transmitted contacts listed (approximately 4.5 more contacts). However, when there was cognitive impairment, the CI's advantage over the standard interview was diminished. Specifically, the CI was superior to the standard interview when no cognitive impairment was present, but performed at a similar level to the standard interview when participants were cognitively impaired. This suggests that participants in the impairment condition may not have had the cognitive resources available that were required to benefit from the CI. Results also suggested that the standard interview generated more precise responses on average than the CI for both the total contacts and droplet-transmitted contacts (precision did not vary as a function of presence or absence of impairment). As predicted, this may 
be attributed to participants in the standard interview condition leaving off their low precision contacts entirely - this would leave them reporting fewer contacts, and on average, more precise contacts. In terms of the overall utility of the responses, the CI was superior to the standard interview when there was no cognitive impairment, because the increase in number of contacts made up for the lower precision of those additional contacts. However, when cognitive impairment was present the contacts generated by the CI were no more useful than in the standard interview.

\section{Limitations}

One potential limitation of the present study was that participants were able to control the extent to which they attended to the impairment task. As such, participants may have paid more attention to the interview and less attention to the task (or vice versa) depending on the interview condition (CI or standard). It could be argued, for example, that the impairment task did not affect participants in the standard condition, because they paid less attention to the task. To ameliorate concerns about differential performance, participants' performance on the impairment task was measured. Results clearly demonstrated no difference in performance on the task as a function of condition, suggesting that the resources dedicated to the cognitive impairment task were similar across both interview types.

There was also a concern that the impairment task would fail to successfully impair retrieval during an interview. The presence of the significant interaction by which the presence of the impairment task reduces the number of contacts generated by the CI compared to when there was no impairment task, however, suggests that the task was difficult enough to have affected reporting. Furthermore, participants in the impairment 
conditions rated the interview as more difficult, reported having expended more mental resources during the interview, and rated recall as less successful than those in the no impairment conditions. This offers further support that the manipulation had the impact intended. Nonetheless, the extent to which the distraction task directly mirrors impairment from acute illness could be debated. As such, future research should compare the CI versus a standard contact tracing interview in participants who are actually ill. Using physically ill participants should serve to more closely replicate the effects of illness in real-world reporting.

\section{GENERAL DISCUSSION}

Contact tracing interviews are an important component of infectious disease control. and are most effective if the lists of potential contacts are comprehensive. However, in order to obtain a list of potential contacts the infected individual must be interviewed. Despite the importance of this interview (arguably one of the most important types of interviews that could be conducted), there is a striking dearth of research on how to most effectively question these individuals to obtain their contacts. The present studies demonstrated that a high quality standard interview generated fewer contacts than an interview aimed at enhancing recall (the CI). The fact that additional contacts are reported in a CI, and not in a high-quality standard interview, disturbingly suggests that some forgetting is taking place during a typical contact tracing interview. Unfortunately, failure to report exhaustively in the standard contact tracing interview may have devastating effects. Unreported contacts might infect many others and/or miss seeking necessary treatment themselves. 
Encouragingly, results suggested that the CI was effective in increasing the number of contacts compared to the standard interview, at least when cognitive impairment was not present. As will be discussed, these findings have clear implications for conducting contact tracing interviews in the real world. In addition to the practical implications, results surrounding the null findings in Study One, cognitive impairment in Study Two, and the measurement of precision and overall utility in both studies, are also theoretically informative and add to the literature on the CI in general.

\section{Null Findings in Study One}

While Study Two clearly demonstrated the superiority of the CI compared to the standard interview, the results from Study One were less clear, and prompted questions regarding why the effect was not found as expected. We suspect that the null findings were a result of a lack of power to detect effects. Indeed, in Study Two, which had a larger sample, the difference between the CI and standard interview was significant. This implies that the increase in recall of physical contacts is possible, but requires more statistical power to uncover the effect.

The sample used in the present study, however, was based on past CI research, which tends to find large effects. This is another way to say that a typical CI study would have likely found differences between a CI and standard interview using the sample size implemented in Study One (and have in fact done so in the past; Memon, Meissner, \& Fraser, 2010). However, we were nonetheless unable to find a difference in the number of droplet-transmitted contacts provided. This inability to find effects, suggests that there are likely some interesting theoretical and/or practical differences between the present study and past CI research, which may explain this discrepancy. 
First, participants were asked to remember people with whom they had close or intimate physical contact - a fairly easy task. Imagine being asked to list the people who you physically touched over the past three days. Now, imagine instead being asked to list every person you saw over the past three days (merely in passing or physical contact). Providing a list of the former is inherently easier than attempting to come up with every face you may have seen. Physical contact not only provides for a more salient memory, it is also likely that the people you physically touched are people you know extremely well. As a result, these contacts are remembered with more ease than unfamiliar strangers. Because listing physical contacts over a three day period is a relatively "easy task," there is less need to provide extra cues for retrieval. In other words, CI mnemonics such as reverse order or varied retrieval are less able to increase the number of contacts listed. In fact, results from Study One suggested that the physical contacts could be remembered with minimal help (i.e., in the standard interview). However, increasing the difficulty of the task should allow the CI more room to be effective (e.g., remembering a list of all persons encountered regardless of physical contact, or a list of contacts from a week ago instead of the past three days).

Second, there is a fairly low ceiling on the number of actual physical contacts a person tends to have and can therefore report. In a typical CI experiment participants view some simulation of a crime and are asked to recall as many details as possible. In recalling the criminal event, there is an almost infinite number of details a witness can provide. When the to-be-remembered stimulus is rich in details, the CI works to help witnesses remember more additional details compared to the standard interview. However, as suggested by Study One, when the range of details to-be-remembered is 
restricted, the CI has a relatively small effect. As a result, in order to detect effects when the number of details to be remembered is limited, more statistical power is required compared to a typical CI study. The fact that there was a benefit of the CI over the standard interview in Study One when considering total contacts (which is a less limited pool of contacts) provides support for this logic.

Third, in a typical CI study every participant watches and recalls the same event. However, in the present studies, participants reported on autobiographical events that differed between participants. Thus, there is a great amount of variability in what the participant could recall. As a result of describing different events, there was likely a larger amount of variability in the information reported in the present studies compared to a typical CI study.

Finally, a major difference between this research and some of the typical CI research is the quality of the standard interview. There is no systematic data on how the majority of epidemiological interviews are conducted (e.g., are only open-ended questions used, are participants repeatedly prompted?). To provide the most conservative comparison of a contact tracing interview to the CI, the standard interview was developed to represent a high-quality contact tracing interview. This high-quality interview even incorporated inadvertently some elements of a CI. For example, participants went through their circle of contacts and then discussed whom they had encountered. This arguably constitutes varying retrieval strategies. Furthermore, primarily open-ended questions were used. Although much recent CI research uses high-quality comparison interviews (e.g., the FLETC five-step; e.g., Rivard, Fisher, Robertson, \& Hirn Mueller, 2014), there are some studies in which the comparison interview is merely one open- 
ended prompt. For instance, in the study assessing the effects of the CI on food histories, one open-ended prompt served as the primary comparison interview (Fisher \& Quigley, 1992). It is highly likely that if a very basic, one-prompt standard interview had been used in Study One, there would have been larger effects of the CI over a standard interview, even when using the typical CI sample size. We expect that the high-quality comparison interview compounded with the other unique obstacles of reporting physical contacts (e.g., easy to remember, low ceiling, little room for improvement) likely contributed to the lack of a statistical difference between the CI and standard interview in Study One.

\section{Novel Additions to CI Literature}

These are the first known studies to date to examine the effects of the CI compared to an infectious disease contact tracing interview. In addition to demonstrating the successful application of the CI to contact tracing, several aspects of the research, i.e., manipulation of cognitive impairment at retrieval, and inclusion of average precision and overall utility make this research novel.

Cognitive Impairment. Perhaps the most novel aspect of this research is the introduction of a cognitive impairment task during retrieval. In fact, to the author's knowledge this is the first study on the $\mathrm{CI}$ to implement a distraction task during reporting. Results suggested that the CI yielded more contacts in the no impairment condition, but was no better than the standard interview in conditions when impairment was present. Reporting during a CI is an arguably effortful process requiring sometimesintense concentration/mental effort. For example, participants are tasked with generating an extremely detailed narration sometimes using complex mnemonics such as reverse 
order (in the present studies) or change-perspective (in other studies). Thus, a distraction task aimed at consuming working memory resources and consequently the ability to allocate mental effort to the interview, effectively prevented the interviewee from fully engaging in the CI. The standard interview, however, seemed to take less effort to complete. Questions were more targeted in a standard interview than in the CI and required less self-generation than a CI. As such, when impairment was applied, the task affected the $\mathrm{CI}$ only, resulting in no difference between the $\mathrm{CI}$ and standard interview.

Additionally, it seems likely that the additional contacts provided by the CI were generally harder to remember than those that were provided across both the standard interview and the $\mathrm{CI}$ (evidenced by the less precise responses in the $\mathrm{CI}$ on average compared to the standard interview). Thus, when impairment is applied to both interviews, the standard interview remains the same, because the difficult contacts would be left out regardless of impairment. However, for the CI, the cognitive impairment interferes with remembering these difficult-to-remember contacts, resulting in no additional contacts generated by the CI compared to the standard interview. Interestingly, in the present studies participants did not perceive the CI to be more difficult to complete than the standard interview. In fact, when the impairment task was completed, the standard interview was rated as more difficult to complete and the contacts remembered less successfully than the CI. We suspect that participants were aware that the CI mnemonics were cognitively demanding, but felt as if the mnemonics were increasing the number of contacts they could recall, leading to lower ratings of difficulty remembering and higher ratings of success. In reality, however, the CI generated no more contacts than the standard interview when the impairment task was completed. This suggests some 
disconnect between subjective ratings of difficulty and success at remembering, and the actual number of contacts reported. Future research should examine whether the $\mathrm{CI}$ is more cognitively demanding than the standard interview, and explore whether there is a disconnect between participants' ratings of difficulty and how much they remember.

It may be that, in a study in which there are an almost infinite number of potential details to report, the CI generates the same details as the standard interview, but also increases both details that are a) more difficult to retrieve and b) details that were omitted in the standard interview because of the sheer volume of details to report or the witness's misunderstanding of the level of detail expected. Thus, when the ceiling of number of details to report is high (e.g., reporting details of a crime video) cognitive impairment may reduce the ability of the CI to generate those more difficult to remember details. However, in this "high ceiling" case, the CI may still maintain the ability to overcome the general omission of details in the standard interview that are left out, not because they are more difficult to remember, but because the witness does not realize the level of detail expected. As a result, in a typical CI study where the ceiling of number of details to report is high, there may be less of an effect of impairment on the total number of details generated by a CI. This hypothesis may be supported by the fact that previous research found no effect of intellectual disability on reporting during a CI - all of these studies involved reporting on an event with an immense number of to-be-remembered details. Future research should attempt to classify the additional information generated by the CI compared to the standard interview (e.g., is the extra information provided by the CI information that was poorly encoded, or information that would not "typically" be 
reported in an interview, or information that is non-schematic for that particular event, or is there no describable pattern?).

Results also suggested that impairment increased the length of the standard interview compared to when no impairment was present, but made no difference in the length of the CI. It may be that the participants who received the impairment task in the standard interview compensated by more slowly responding to questions compared to when no impairment task was provided, thus preventing a decrease in the amount of contacts reported as a result of impairment. In the CI condition participants did not seem to compensate the same way - their interviews were not longer in the impairment task condition. Rather they "compensated" for the impairment by providing fewer contacts.

The results involving cognitive impairment tend to run counter to what has been found in research on the CI for ID adults. However, we would caution against generalizing results of the cognitive impairment task to adults with an intellectual disability. There are several differences between the impairment task used and an actual intellectual disability. The task used was likely more targeted at impairing the working memory resources necessary for retrieval than an intellectual disability. Furthermore, intellectual disabilities examined in research tend to be mild and vary widely in level of impairment (e.g., Bull, 2010). It is therefore possible that the distraction task, standardized across participants, was much more effective at inhibiting recall during a CI than an intellectual disability. As noted previously, another difference is that the impairment task was temporary- only taking place during retrieval. Encoding and storage processes were not impacted. In contrast, someone with an intellectual disability has the same limitations at encoding, storage, and retrieval. 
Precision. To the author's knowledge, this is the first study to date to examine how the CI versus a standard interview affects the precision of the provided responses. Conventionally, in CI research the responses to an interview are scored in terms of quantity and accuracy. The examination of the precision of interview response is a relatively unstudied area of study (but see, e.g., Evans \& Fisher, 2011). Precision is typically conceptualized as the grain-size or level of specificity at which a response is provided. For example, if a witness described a getaway car as "a blue truck with large silver rims" the response would be considered more precise than if the witness described the car as "a dark truck with rims." In the present studies precision was conceptualized slightly differently; to make the results practically relevant precision was operationalized as the ease with which any given contact could be identified and/or located. Using this conceptualization, the maximum level of precision was reached when a first and last name was provided. Note that if a participant listed a first and last name, and also a detailed description of the person, the score of precision was no higher than if just the names were provided. It is important to note that this differs from the traditional conceptualization of precision as the level of specificity of a response. If contacts from the present study were scored for precision as more traditionally conceptualized, a first and last name along with a list of specific details would be scored as more precise than just a first and last name.

We predicted that the CI would generate all the same contacts, with the same level of precision as the standard interview (i.e., close contacts like family, friends and significant others). However, the CI was also expected to generate additional contacts, at a lower average level of precision, than what was provided by the standard interview (i.e., 
more difficult to remember contacts like classmates and coworkers). Thus, it was hypothesized that because the additional contacts provided in the CI would tend to be lower in precision (i.e., harder to find), the average precision score for the CI would be lower than the score in the standard interview. The results supported our hypothesis. Nonetheless, it must be acknowledged that because the research employed a between subjects design it did not directly test the proposed underlying process (i.e., the CI generated the same contacts as the standard interview, plus other less precise contacts).

It is, perhaps, reassuring that the responses to a standard interview were more precise on average than those in the CI (in Study Two). This suggests that even though there might be some important physical contacts missing when a standard interview is used, the contacts that are reported in the standard interview are more likely to be easily found, and may even be at higher risk of infection (e.g., more likely to include only family, or close friends), than the extra contacts provided in the CI. Nevertheless, in some cases the extra contacts reported in the CI could mean the difference between life and death. As such, the advantage of an average precision score for the standard interview is outweighed by the overall utility of providing more contacts, regardless of level of precision.

We expect the findings related to precision and interview type to extend to research on the CI versus a standard interview when even a more traditional measure of precision (i.e., level of specificity) is used. The CI tends to increase the number of details provided, and as such, it is likely that much of the extra information generated is lower in precision. However, future research should examine whether the results found here hold when a traditional measure of precision is used. 
Utility. Although the average precision of responses was higher in the standard interview, the overall utility of responses was higher in the CI (in Study Two). This result was likely driven by the fact that the CI generated more contacts than the standard interview. Since more contacts were provided, the list had more overall utility than a standard interview. Interestingly, CI research has been criticized for an inability to demonstrate that the extra details provided in a CI are actually useful to an investigation (Memon, Meissner, \& Fraser, 2010). In the present study however, the extra contacts listed (especially the extra droplet-transmitted names) are important. Specifically, even though the droplet-transmitted contacts generated by the CI were less precise on average, these contacts were still classified as high-risk for the contracting and spreading of the infectious disease. This suggests that the extra details provided by the CI were, in fact, useful. Of course, their usefulness is diminished if they are provided with a low level of precision and are difficult or impossible to identify and locate.

\section{Application to Alibi Statements}

Another context in which people are asked to list places visited and people encountered is generating true alibi statements to a crime (e.g., Allison, Michael, Mathews, \& Overman, 2011; Crozier, Strange, \& Loftus, 2017; Culhane, Hosch, \& Kehn, 2008; Culhane et al., 2013; Olson \& Charman, 2012). In fact, there are many different facets of providing an alibi that are similar to reporting during contact tracing. For example, when a person provides an alibi he or she attempts to remember events that were incidentally encoded (i.e., not intentionally remembered). In eyewitness memory research the participant is often aware that the information presented is likely to be requested later. However, in generating an alibi, the individual is typically recalling 
everyday events for which he or she did not pay attention and therefore has difficulty remembering later (Crozier, Strange, \& Loftus, 2017). Reporting an alibi is also unique to other types of reporting because it asks individuals to remember autobiographical events, which often occur repeatedly (i.e., a person trying to remember details about one specific instance (e.g., driving to school last Tuesday) amongst all other instances (e.g., driving to school on Thursday, or on the Tuesday before)). Research has suggested that remembering repeated events is difficult because a) witnesses tend to remember only the "gist" (what usually happens) of the event (e.g., Connely \& Lindsay, 2001; cited in Willen, Granhag, Stromwall, \& Fisher, 2015) and b) witnesses struggle to determine the particular source of the memory (e.g., during which of the drives to school did the individual call his or her sister; Johnson, Hashtroudi, \& Lindsay, 1993; cited in Willen,

Granhag, Stromwall, \& Fisher, 2015). Interestingly, the factors that make recall unique to alibi generation compared to traditional interviewing research are very similar to those involved in a contact tracing interview (which also involves incidental autobiographical memory for repeated events). The present research is therefore highly applicable to generating alibis. If a person needs to provide an exhaustive (true) alibi, we recommend the use of a CI in order to improve statement detail.

\section{Practical Implications}

Results of this research have serious implications for interviewing during contact tracing. Most notably, we found that the CI was substantially superior to the standard interview, but only when the interviewee was not experiencing cognitive impairment. We would caution against concluding from these findings that the CI should be avoided or is unnecessary for sick individuals. For example, there may be instances in which patients 
can be interviewed for their contacts before acute symptoms develop, or there may be diseases in which there are few symptoms (e.g., Zika). Thus, the cognitive impairment is irrelevant and the CI would be expected to increase the number of contacts generated. Furthermore, individuals who are identified as contacts by a sick patient, but who are not yet suffering from symptoms themselves, are often interviewed as well. In these cases, no cognitive impairment is expected to be present. There is also likely a great deal of variation in the level of cognitive impairment experienced by patients who are sick. For example, a person with early stages of the flu might not exhibit the same levels of cognitive impairment as someone who is seriously sick with Ebola. As such, a blanket avoidance of the CI when someone is sick would be unadvised. As discussed, however, the participants in the present studies were not sick. Before any concrete conclusions can be made about reporting contacts when sick, research should examine whether the findings from Study Two extend to participants who are actually sick. Furthermore, it may be that a patient with large working memory capacity is not as affected by the cognitive impairment associated with illness as someone with small working memory capacity. Future research should examine whether the effects of cognitive impairment on reporting differ based on an individual's working memory capacity.

There are many practical applications of this research to various diseases. For example, the CI appeared to aid in remembering more obscure contacts than the standard interview. We are therefore optimistic that the CI will be especially helpful during the outbreak of a serious airborne illness in which contacts are often strangers. The CI is also likely to be of particular benefit for illnesses with airborne transmission because there is a much higher ceiling on the number of to-be-reported contacts. As such, there is more 
room for improvement in retrieval. Overall, we expect the CI to have an even greater effect on reporting in an airborne context compared to reporting about a droplettransmited disease.

Another type of disease transmission mechanism, for which the utility of the CI has not been examined, is that of mosquito-borne illnesses. One timely example of such an illness is Zika Virus Disease. Zika is a primarily mosquito-borne illness (via the Aedes aegypti), but is also transmitted sexually. Once a patient is infected with Zika, it is imperative to determine where they have been and with whom they have had sexual contact. This allows officials to isolate where infected mosquitos are most likely to be located, and identify individuals who may be infected with (and transmitting) Zika.

One of the primary mechanisms for controlling the spread of mosquito-borne illnesses is widespread spraying of large areas with truck-mounted insecticides. In a recent study, researchers tested a method in which nurses contacted every patient confirmed to have a dengue infection (a mosquito-borne illness) and interviewed them about the places they had been (e.g., inside personal residences). Only some of the identified places were then sprayed with insecticides, thereby allowing researchers to compare the dengue transmission of sprayed and unsprayed areas. Results suggested that the targeted spraying based on the interview reduced the chances of transmission by up to $96 \%$, which was lauded by researchers as an unprecedented success. As a result, combining the interview with targeted spraying of insecticides was recommended for use in other mosquito-borne illnesses, such as Zika, as well (Vazquez-Prokopec et al., 2017). Because contact tracing is vital to the eradication of these types of illness, the present studies can provide insight into how to most effectively conduct these contact tracing 
interviews. Results suggest that the CI would generate a more exhaustive list of the places visited than a typical interview (although, like with physical contacts, there may be a low ceiling on visited locations, making the potential benefit of the CI limited). As a result of this increase in information, more areas for spraying and potential contacts might be identified, helping stem the further spread of the disease.

\section{Practical Limitations}

There may be situations in which interviewing a patient about his or her contacts is limited by practical constraints (e.g., sick patient cannot spend an extended amount of time on the interview). When using a CI this problem may be even more pronounced. A $\mathrm{CI}$ is likely more difficult for an interviewer to conduct (although we were able to train undergraduate research assistants to conduct the interviews in a short period of time) and is demonstrated to take more time than a standard interview. Nevertheless, contact tracing investigations are generally time-consuming and resource demanding. As such, the extra 20 minutes it might take to conduct a CI instead of a standard interview is insignificant considering the immense time spent on the entire contact tracing investigation.

Although the extra time to conduct a CI may not be of utmost concern, it may still be beneficial to find creative ways to conserve resources. For example, a smartphone application was developed recently to allow potentially infected individuals to systematically input the people with whom they had been in contact (Epi Info viral hemorrhagic fever (VHF) application; Schafer et al., 2016). This should allow individuals, who might otherwise not take part in a contact tracing interview for practical reasons, contribute to contact tracing efforts. In light of the results of the present studies, the completeness of a list provided to written requests from an app is likely insufficient. 
Helpfully, there is research suggesting that a written version of the CI (the "selfadministered interview") can be useful in generating information from a witness (Gabbert, Hope, \& Fisher, 2009; 2012; Hope, Gabbert, \& Fisher, 2011). Future research should address the effectiveness of providing contacts in response to a smartphone application compared to an in-person interview and whether the superiority of the CI over a standard interview still holds when the responses are provided via a smartphone application.

\section{Methodological Limitations}

One limitation of the present research was that the accuracy of responses could not be assessed in either study. As such, there is no way to verify whether the extra contacts generated by the CI were accurate or generated in error. It could be that the CI encouraged participants to list contacts with whom they had not actually had physical contact, thus driving the overall increase in contacts compared to the standard interview. We have reason to expect, however, that the accuracy rates of contacts listed in the CI and standard interview were comparable. Notably, meta-analyses examining data across all published CI research has demonstrated no difference in the accuracy rates of the CI compared to standard interviews (Kohnken, Milne, Memon, \& Bull, 1999; Memon, Meissner, \& Fraser, 2010). These findings are likely explained by the fact that errors of commission are typically rare when participants are provided the opportunity to withhold responses based on their confidence in the accuracy of that response (e.g., Koriat \& Goldsmith, 1996). Because the ability to control the accuracy of responses is maximized when open-ended questions are used, as in the present studies, it is likely that the accuracy rates were high (e.g., Fisher et al., 2012). 
Note also that, in a contact tracing interview, it is typically more valuable to receive an exhaustive list with a small number of contacts listed in error, than to receive a more conservative list with no inaccurate contacts. Thus, accuracy was not considered in the present studies and instead an emphasis was placed on reporting as many contacts as possible in as naturalistic a way as possible. There are methods that can be used that would allow accuracy to be determined, but they come at the expense of losing ecological validity. For example, consider a "speed-dating paradigm" during which participants interact with numerous other participants in a controlled environment and are asked later to report the people with whom they interacted. In this type of paradigm, researchers can keep track of every interaction and therefore the accuracy of every reported contact. However, every contact listed would likely be a stranger with whom the participant interacted with only once. Providing a list of strangers is less naturalistic than the method used in the present studies, and might result in different findings. Future research should creatively test the differences between the CI and standard interview in a paradigm in which accuracy can be assessed, while also balancing ecological validity (e.g., participant wears a video camera to a social event). Future research could also use a paradigm in which participants recall their contacts in an interview, but after the interview phase are allowed to use their calendars, phones, or social media to report any contacts that may have been missed, and to corrobate the accuracy of the contacts provided in the interview. This would serve as a way to both test the completeness of the list of contacts provided, and potentially assess the accuracy of some of the already provided contacts. 


\section{Conclusion}

Studies One and Two were important first steps in assessing the differences between the CI and a more typical contact tracing interview. Results encouragingly suggested that the number of reported contacts can be increased via the CI. As such, we recommend that the $\mathrm{CI}$ be considered as an alternative to the standard contact tracing procedure. We believe that this area of research is ripe for additional research. Perhaps most importantly, future research should examine the effects of the CI versus the standard interview in a paradigm in which witnesses are actually ill. Furthermore, the present study compared the CI to a high-quality contact tracing interview, which may not be representative of a more typical interview conducted by an epidemiologist. Future research should also examine the effects of the CI compared to a more representative contact tracing interview. 


\section{REFERENCES}

Allison, M., Michael, S. W., Mathews, K. R., \& Overman, A. A. (2011). Brief report: Narrative qualities and perceptions of generated alibis. North American Journal of Psychology, 13(3), 359-365.

Anderson, M. C., Bjork, R. A., \& Bjork, E. L. (1994). Remembering can cause forgetting: retrieval dynamics in long-term memory. Journal of Experimental Psychology: Learning, Memory, and Cognition, 20(5), 1063.

Andre, M., Ijaz, K., Tillinghast, J. D., Krebs, V. E., Diem, L. A., Metchock, B., Crisp, T, \& McElroy, P. D. (2007). Transmission network analysis to complement routine tuberculosis contact investigations. American journal of public health, 97(3), 470.

Armbruster, B., \& Brandeau, M. L. (2007). Contact tracing to control infectious disease: when enough is enough. Health care management science, 10(4), 341-355.

Baddeley, A. D. (1986). Working memory. Oxford England: Clarendon Press.

Baddeley, A. (2000). The episodic buffer: a new component of working memory?. Trends in Cognitive Sciences, 4(11), 417-423.

Bartlett, F. C. (1932/1935). Remembering: A study in experimental and social psychology. New York: Cambridge University Press [reprint].

Bartlett, F. C., \& Burt, C. (1933). Remembering: A study in experimental and social psychology. British Journal of Educational Psychology, 3(2), 187-192.

Baldauf, D., Burgard, E., Wittmann, M. (2009). Time perception as a workload measure in simulated car driving. Applied Ergonomics, 40, 929-935.

Bransford, J. D., \& Johnson, M. K. (1973). Considerations of some problems of comprehension. In W. Chase (Ed.), Visual information processing (pp. 383-438). New York: Academic Press.

Brewer, D. D., Garrett, S. B., \& Kulasingam, S. (1999). Forgetting as a cause of incomplete reporting of sexual and drug injection partners. Sexually transmitted diseases, 26(3), 166-176.

Brewer, D. D. (2002). Supplementary interviewing techniques to maximize output in free listing tasks. Field Methods, 14, 108-118.

Brewer, D. D., Garrett, S. B., \& Rinaldi, G. (2002). Free listed items are effective cues for eliciting additional items in semantic domains. Applied Cognitive Psychology, $16,343-358$. 
Brewer, D. D., \& Garrett, S. B. (2001). Evaluation of interviewing techniques to enhance recall of sexual and drug injection partners. Sexually Transmitted Diseases, 28, 666-677.

Brewer, D. D., Potterat, J. J., Muth, S. Q., Malone, P. Z., Montoya, P., Green, D. L., ... \& Cox, P. A. (2005). Randomized Trial of Supplementary Interviewing Techniques to Enhance Recall of Sexual Partners in Contact Interviews*.Sexually transmitted diseases, 32(3), 189-193

Brock, P., Fisher, R. P., \& Cutler, B. L. (1999). Examining the cognitive interview in a double-test paradigm. Psychology, Crime, \& Law, 5, 29-45. doi 10.1080/10683169908414992

Brown, S. C., \& Craik, F. I. (2000). Encoding and retrieval of information. In E Tulving \& F. I. M. Craik (Eds.), The Oxford handbook of memory (pp. 93-107). New York: Oxford University Press.

Brown, C. L. \& Geiselman, R. E. (1990). Eyewitness testimony of mentally retarded: Effect of the cognitive interview. Journal of Police and Criminal Psychology, 6, 14-22.

Bull, R. (2010). The investigative interviewing of children and other vulnerable witnesses: Psychological research and working/professional practice. Legal and Criminological Psychology, 15(1), 5-23.

Cannell, C. F. (1959-1970). A summary of research studies of interviewing methodology. Rockville, MD: National Center for Health Statistics, DHEW Pub. No. (HRA) 771343. .

Centers for Disease Control and Prevention. (2000) Community TB control: Identification of persons who have clinically active TB. Retrieved from http://www.cdc.gov/nchstp/tb/pubs/corecurr/Chapter10/Chapter_10_Identification .htm.

Centers for Disease Control and Prevention. (2002) Sexually transmitted diseases treatment guidelines. Retrieved from http://www.cdc.gov/STD/treatment/12002TG.htm.

Centers for Disease Control and Prevention. (2014, May 14). Infectious Disease. Retrieved from http://www.cdc.gov/nchs/fastats/infectious-disease.htm

Cepeda, N. J., Pashler, H., Vul, E., Wixted, J. T., \& Rohrer, D. (2006). Distributed practice in verbal recall tasks: A review and quantitative synthesis. Psychological bulletin, 132(3), 354. 
Chapple, A. (1999). The use of telephone interviewing for qualitative research. Nurse Researcher, 6(3), 85.

Clarke, J. (1998). Contact tracing for chlamydia: data on effectiveness. International journal of STD \& AIDS, 9(4), 187-191.

Clifford, B. R., \& George, R. (1996). A field evaluation of training in three methods of witness/victim investigative interviewing. Psychology, Crime and Law, 2(3), 231248.

Cohen, G., \& Faulkner, D. (1989). Age differences in source forgetting: Effects on reality monitoring and on eyewitness testimony. Psychology and Aging, 4(1), 10.

Connolly, D. A., \& Lindsay, D. S. (2001). The influence of suggestions on children's reports of a unique experience versus an instance of a repeated experience. Applied Cognitive Psychology, 15(2), 205-223.

Craik, F. I., Govoni, R., Naveh-Benjamin, M., \& Anderson, N. D. (1996). The effects of divided attention on encoding and retrieval processes in human memory. Journal of Experimental Psychology: General, 125(2), 159.

Craik, F. I., \& Tulving, E. (1975). Depth of processing and the retention of words in episodic memory. Journal of experimental Psychology: general, 104(3), 268.

Crozier, W. E., Strange, D., \& Loftus, E. F. (2017). Memory errors in alibi generation: How an alibi can turn against us. Behavioral Sciences \& the Law, doi:http://dx.doi.org.ezproxy.fiu.edu/10.1002/bs1.2273

Culhane, S. E., Kehn, A., Horgan, A. J., Meissner, C. A., Hosch, H. M., \& Wodahl, E. J. (2013). Generation and detection of true and false alibi statements. Psychiatry, Psychology and Law, 20(4), 619-638.

Culhane, S. E., Hosch, H. M., \& Kehn, A. (2008). Alibi generation: Data from US Hispanics and US non-Hispanic whites. Journal of Ethnicity in Criminal Justice, 6(3), 177-199.

Cvejic, E., Lemon, J., Hickie, I. B., Lloyd, A. R., \& Vollmer-Conna, U. (2014). Neurocognitive disturbances associated with acute infectious mononucleosis, Ross River fever and Q fever: A preliminary investigation of inflammatory and genetic correlates. Brain, behavior, and immunity, 36, 207-214.

Decker, M. D., Booth, A. L., Hutcheson, R. H., \& Schaffner, W. (1986). Validity of food consumption histories in a foodborne outbreak investigation. American journal of epidemiology, 124(5), 859-863. 
Eames, K. T., \& Keeling, M. J. (2003). Contact tracing and disease control. Proceedings of the Royal Society of London. Series B: Biological Sciences, 270(1533), 25652571 .

Ebbinghaus H (1885) Über das Gedächtnis. Leipzig: Dunker.

Evans, J. R., \& Fisher, R. P. (2011). Eyewitness memory: Balancing the accuracy, precision and quantity of information through metacognitive monitoring and control. Applied Cognitive Psychology, 25(3), 501-508.

Faul, F., Erdfelder, E., Lang, A. G., \& Buchner, A. (2007). G* Power 3: A flexible statistical power analysis program for the social, behavioral, and biomedical sciences. Behavior Research Methods, 39(2), 175-191.

Fisher, R. P. (1995). Interviewing victims and witnesses of crime. Psychology, Public Policy, and Law, 1(4), 732-764. doi:http://dx.doi.org/10.1037/1076-8971.1.4.732

Fisher, R. P., Falkner, K. L., Trevisan, M., \& McCauley, M. R. (2000). Adapting the Cognitive Interview to enhance long-term (35-years) recall of psychical activities. Journal of Applied Psychology, 85, 180-189. doi: 10.1037//0021-9010.85.2.180.

Fisher, R. P., Geiselman, R. E., \& Amador, M. (1989). Field test of the cognitive interview: Enhancing the recollection of the actual victims and witnesses of crime. Journal of Applied Psychology, 74(5), 722.

Fisher, R. P., \& Geiselman, R. E. (1992). Memory enhancing techniques for investigative interviewing: The Cognitive Interview. Springfield, IL: Charles C. Thomas.

Fisher, R. P., Milne, R., \& Bull, R. (2011). Interviewing cooperative witnesses. Current Directions in Psychological Science, 20(1), 16-19.

Fisher, R. P., Ross, S. J., \& Cahill, B. S. (2010). Interviewing witnesses and victims. Forensic psychology in context: Nordic and international approaches. (pp. 56-74) Willan Publishing, Devon.

Fisher, R. P., \& Schreiber, N. (2007). Interviewing protocols to improve eyewitness memory. In M. Toglia, et al. (Eds.), The handbook for eyewitness psychology, Vol. I: Memory for events (pp. 53-80). Mahwah, NJ: Lawrence Erlbaum

Fisher, R. P., \& Quigley, K. L. (1992). Applying cognitive theory in public health investigations: Enhancing food recall with the cognitive interview. In J. M. Tanur (Ed.), Questions about questions: Inquiries into the cognitive bases of surveys (pp. 154-169). New York: Russell Sage Foundation

Foster, J. L., Shipstead, Z., Harrison, T. L., Hicks, K. L., Redick, T. S., \& Engle, R. W. (2015). Shortened complex span tasks can reliably measure working memory capacity. Memory \& Cognition, 43(2), 226-236. 
Gabbert, F., Hope, L., \& Fisher, R. P. (2009). Protecting eyewitness evidence: Examining the efficacy of a self-administered interview tool. Law and Human Behavior, 33(4), 298-307.

Gabbert, F., Hope, L., Fisher, R. P., \& Jamieson, K. (2012). Protecting against misleading post-event information with a self-administered interview. Applied Cognitive Psychology, 26(4), 568-575.

Geiselman, R. E., Fisher, R. P., MacKinnon, D. P., \& Holland, H. L. (1986). Enhancement of eyewitness memory with the cognitive interview. The American journal of psychology, 385-401.

Gilbert, J. A., \& Fisher, R. P. (2006). The effects of varied retrieval cues on reminiscence in eyewitness memory. Applied Cognitive Psychology, 20(6), 723-739.

Grant, R. C., Carswell, C. M., Lio, C. H., \& Seales, W. B. (2013). Measuring surgeons' mental workload with a time-based secondary task. Ergonomics in Design, 21(1), 7-11.

Grant, R.C., Carswell, C.M., Lio, C.H., Seales, W.B., and Clark, D. (2009). Verbal Time Production as a Secondary Task: Which Metrics and Target Intervals are Most Sensitive to Workload for Fine Motor Laparoscopic Training Tasks? Human Factors and Ergonomics Society Annual Meeting Proceedings, 18, 1191-1195.

Griffiths, A., \& Milne, R. (2010). The application of cognitive interview techniques as part of an investigation. Consultancy and advising in forensic practice: Empirical and practical guidelines, 71-90.

Hershkowitz, I., Lamb, M. E., \& Katz, C. (2014). Allegation rates in forensic child abuse investigations: Comparing the revised and standard NICHD protocols. Psychology, Public Policy, and Law, 20(3), 336.

Hershkowitz, I., \& Terner, A. (2007). The effects of repeated interviewing on children's forensic statements of sexual abuse. Applied Cognitive Psychology, 21(9), 11311143 .

Holliday, R. E., Brainerd, C. J., Reyna, V. F., \& Humphries, J. E. (2009). The cognitive interview: Research and practice across the lifespan. R. Bull, T. Valentine, \& T. Williamson (Eds.), Handbook of the psychology of investigative interviewing: Current developments and future directions, 137-160.

Hope, L., Gabbert, F., \& Fisher, R. P. (2011). From laboratory to the street: Capturing witness memory using the Self-Administered Interview. Legal and criminological psychology, 16(2), 211-226.

Johnson, M. K., Hashtroudi, S., \& Lindsay, D. S. (1993). Source monitoring. Psychological bulletin, 114(1), 3. 
Kebbell, M. R., Milne, R., \& Wagstaff, G. F. (1999). The cognitive interview: A survey of its forensic effectiveness. Psychology, Crime and Law, 5(1-2), 101-115.

Kebbell, M. R., \& Milne, R. (1998). Police officers' perceptions of eyewitness performance in forensic investigations. The Journal of Social Psychology,138(3), 323-330.

Kiss, I. Z., Green, D. M., \& Kao, R. R. (2005). Disease contact tracing in random and clustered networks. Proceedings of the Royal Society of London B: Biological Sciences, 272(1570), 1407-1414.

Klein, S. B., \& Kihlstrom, J. F. (1986). Elaboration, organization, and the self-reference effect in memory. Journal of Experimental Psychology: General,115(1), 26.

Köhnken, G., Milne, R., Memon, A., \& Bull, R. (1999). The cognitive interview: A metaanalysis. Psychology, Crime and Law, 5(1-2), 3-27.

Klatzky, R. L. (1980). Human memory: Structures and processes. San Francisco: Freeman.

Krall, E. A., \& Dwyer, J. T. (1987). Validity of a food frequency questionnaire and a food diary in a short-term recall situations. Journal of the American Dietetic Association, 73(2).

La Rooy, D., \& Lamb, M. E. (2011). What happens when interviewers ask repeated questions in forensic interviews with children alleging abuse?. Journal of Police and Criminal Psychology, 26(1), 20-25.

La Rooy, D. L., Pipe, M. E., \& Murray, J. E. (2005). Reminiscence and hypermnesia in children's eyewitness memory. Journal of Experimental Child Psychology, 90(3), 235254.

Lindsay, D. S., Allen, B. P., Chan, J. C., \& Dahl, L. C. (2004). Eyewitness suggestibility and source similarity: Intrusions of details from one event into memory reports of another event. Journal of Memory and Language, 50(1), 96-111.

Lipsitch, M., Cohen, T., Cooper, B., Robins, J. M., Ma, S., James, L., ... \& Fisman, D. (2003). Transmission dynamics and control of severe acute respiratory syndrome. Science, 300(5627), 1966-1970.

Liu, Y. \& Wickens, C. D. (1994). Mental workload and cognitive task automaticity: an evaluation of subjective and time estimation metrics. Ergonomics, 37(11), 18431854. 
Loftus, E. F. (1975). Leading questions and the eyewitness report. Cognitive Psychology, $7(4), 560-572$.

Loftus, E. F., \& Zanni, G. (1975). Eyewitness testimony: The influence of the wording of a question. Bulletin of the Psychonomic Society, 5(1), 86-88.

MacLeod, M. (2002). Retrieval-induced forgetting in eyewitness memory: forgetting as a consequence of remembering. Applied Cognitive Psychology, 16(2), 135-149.

Mann, J.A. (1981) A prospective study of response error in food history questionnaires. American Journal of Public Health 70, 401-412.

McClelland, G. H. (2000). Nasty data. Handbook of research methods in social psychology, 393-411.

Melton, A. W. (1963). Implications of short-term memory for a general theory of memory. Journal of Memory and Language, 2(1), 1.

Memon, A., Meissner, C. A., \& Fraser, J. (2010). The Cognitive Interview: A metaanalytic review and study space analysis of the past 25 years. Psychology, Public Policy, and Law, 16(4), 340.

Migueles, M., \& García-Bajos, E. (2007). Selective retrieval and induced forgetting in eyewitness memory. Applied Cognitive Psychology, 21(9), 1157-1172.

Milne, R., \& Bull, R. (2001). Interviewing witnesses with learning disabilities for legal purposes. British Journal of Learning Disabilities, 29(3), 93-97.

Milne, R., Clare, I.C.H., \& Bull, R. (1999). Interviewing adults with learning disability with cognitive interview. Psychology, Crime Law, 5, 81-100.

Mitchell, K. J., \& Johnson, M. K. (2000). Source monitoring: Attributing mental experiences. In E Tulving \& F. I. M. Craik (Eds.), The Oxford handbook of memory (pp. 179-195). New York: Oxford University Press.

Molinaro, P., Fisher, R. P., Mosser, A., Satin, G. (2014) Training the trainer on the Cognitive Interview: Translating science into practice. Paper presentation at the National Academy of Sciences, Washington D.C.

Morris, C. D., Bransford, J. D., \& Franks, J. J. (1977). Levels of processing versus transfer appropriate processing. Journal of Verbal Learning and Verbal Behavior, 16(5), 519-533.

Moscovitch, M., \& Craik, F. I. (1976). Depth of processing, retrieval cues, and uniqueness of encoding as factors in recall. Journal of Verbal Learning and Verbal Behavior, 15(4), 447-458. 
Murre, J. M., \& Dros, J. (2015). Replication and analysis of Ebbinghaus' forgetting curve. PloS one, 10(7), e0120644.

Neisser, U. (1967). Cognitive psychology. New York: Appleton-Century-Crofts.

Nelson, T. O. (1971). Savings and forgetting from long-term memory. Journal of Verbal Learning and Verbal Behavior, 10(5), 568-576.

Oeberst, A. (2012). If anything else comes to mind... better keep it to yourself? Delayed recall is discrediting - unjustifiably. Law and Human Behavior, 36(4), 266.

Olson, E. A., \& Charman, S. D. (2012). 'But can you prove it?'-examining the quality of innocent suspects' alibis. Psychology, Crime \& Law, 18(5), 453-471.

Perlman, N. B., Ericson, K. I., Esses, V. M., \& Isaacs, B. J. (1994). The developmentally handicapped witness: Competency as a function of question format. Law and Human Behavior, 18(2), 171.

Pickering, L.K., Baker, C.J., Kimberlin, D.W., Long S.S. (2012). Meninigococcal Infections. In Red Book; American Academy of Pediatrics, 500-509.

Pirolli, P. L., \& Anderson, J. R. (1985). The role of practice in fact retrieval. Journal of experimental psychology: Learning, memory, and cognition, 11(1), 136.

Porco, T. C., Holbrook, K. A., Fernyak, S. E., Portnoy, D. L., Reiter, R., \& Aragón, T. J. (2004). Logistics of community smallpox control through contact tracing and ring vaccination: a stochastic network model. BMC public health, 4(1), 1.

Potterat, J. J. (1997). Contact tracing's price is not its value. Sexually transmitted Diseases, 24(9), 519-521.

Prieto, L., Lamarca, R., Casado, A., \& Alonso, J. (1997). The evaluation of agreement on continuous variables by the intraclass correlation coefficient. Journal of epidemiology and community health, 51(5), 579-581.

Rivard, J. R., Fisher, R. P., Robertson, B., \& Hirn Mueller, D. (2014). Testing the Cognitive Interview with professional interviewers: Enhancing recall of specific details of recurring events. Applied Cognitive Psychology, 28(6), 917-925.

Roebers, C. M., \& Schneider, W. (2000). The impact of misleading questions on eyewitness memory in children and adults. Applied Cognitive Psychology, 14(6), 509-526.

Roediger, H.L., McDermott, K.B., \& Goff, L.M. (1997). Recovery of true and false memories: Paradoxical effects of repeated testing. In M.A. Conway (Ed.), 
Recovered memories and false memories (pp. 118_149). Oxford: Oxford University Press.

Roediger, H. L., \& Payne, D. G. (1982). Hypermnesia: the role of repeated testing. Journal of Experimental Psychology: Learning, Memory, and Cognition, 8(1), 66.

Scarborough, D. L., Cortese, C., \& Scarborough, H. S. (1977). Frequency and repetition effects in lexical memory. Journal of Experimental Psychology: Human perception and performance, 3(1), 1.

Schacter, D. L. (1999). The seven sins of memory: Insights from psychology and cognitive neuroscience. American Psychologist, 54(3), 182.

Schacter, D. L., Guerin, S. A., \& Jacques, P. L. S. (2011). Memory distortion: An adaptive perspective. Trends in cognitive sciences, 15(10), 467-474.

Schafer, I. J., Knudsen, E., McNamara, L. A., Agnihotri, S., Rollin, P. E., \& Islam, A. (2016). The Epi Info Viral Hemorrhagic Fever (VHF) Application: A Resource for Outbreak Data Management and Contact Tracing in the 2014-2016 West Africa Ebola Epidemic. Journal of Infectious Diseases, 3(214), 122-136.

Shaw, J. S., Bjork, R. A., \& Handal, A. (1995). Retrieval-induced forgetting in an eyewitness-memory paradigm. Psychonomic Bulletin \& Review, 2(2), 249-253.

Sifferlin, A. (2015, March 12). Ebola deaths top 10,000. Retrieved from http://time.com/3742986/ebola-deaths-10000/

Smith, A. P. (2012). Effects of the common cold on mood, psychomotor performance, the encoding of new information, speed of working memory and semantic processing. Brain, behavior, and immunity, 26(7), 1072-1076.

Smith, A. P. (2013). Twenty-five years of research on the behavioural malaise associated with influenza and the common cold. Psychoneuroendocrinology, 38(6), 744-751.

Smith, A. P., \& Jamson, S. (2012). An investigation of the effects of the common cold on simulated driving performance and detection of collisions: a laboratory study. BMJ open, 2(4).

Symons, C. S., \& Johnson, B. T. (1997). The self-reference effect in memory: a metaanalysis. Psychological bulletin, 121(3), 371.

Tulving, E. (1974). Cue-Dependent Forgetting: When we forget something we once knew, it does not necessarily mean that the memory trace has been lost; it may only be inaccessible. American Scientist, 62(1), 74-82.

Tulving, E., \& Osler, S. (1968). Effectiveness of retrieval cues in memory for words. Journal of Experimental Psychology, 77(4), 593. 
Tulving, E., \& Pearlstone, Z. (1966). Availability versus accessibility of information in memory for words. Journal of Verbal Learning and Verbal Behavior, 5(4), 381391.

Tulving, E., \& Thomson, D. M. (1973). Encoding specificity and retrieval processes in episodic memory. Psychological review, 80(5), 352.

Tulving, E., \& Watkins, M. J. (1975). Structure of memory traces. Psychological Review, 82(4), 261.

Turtle, J.W., \& Yuille, J.C. (1994). Lost but not forgotten details: Repeated eyewitness recall leads to reminiscence but not hypermnesia. Journal of Applied Psychology, $79,260-271$.

Unsworth, N., Heitz, R. P., Schrock, J. C., \& Engle, R. W. (2005). An automated version of the operation span task. Behavior Research Methods, 37, 498-505.

Vallano, J. P., \& Compo, N. S. (2011). A comfortable witness is a good witness: Rapport-building and susceptibility to misinformation in an investigative mockcrime interview. Applied Cognitive Psychology, 25(6), 960-970.

Vallano, J. P., \& Schreiber Compo, N. (2015). Rapport-building with cooperative witnesses and criminal suspects: A theoretical and empirical review. Psychology, Public Policy, and Law, 21(1), 85.

Vazquez-Prokopec, G.M., Montgomery, B. L., Horne, P. Clennon, J. A., Ritchie, S.A. (2017). Combining contact tracing with targeted indoor residual spraying significantly reduces dengue transmission. Science Advances, 3(2). DOI: 10.1126/sciadv. 1602024.

Vicente, K. J., \& Brewer, W. F. (1993). Reconstructive remembering of the scientific literature. Cognition, 46(2), 101-128.

Vredeveldt, A., Baddeley, A. D., \& Hitch, G. J. (2014). The effectiveness of eye-closure in repeated interviews. Legal and Criminological Psychology, 19(2), 282-295.

Vredeveldt, A., Hitch, G. J., \& Baddeley, A. D. (2011). Eyeclosure helps memory by reducing cognitive load and enhancing visualisation. Memory \& Cognition, 39(7), 1253-1263.

Vredeveldt, A., \& Penrod, S. D. (2013). Eye-closure improves memory for a witnessed event under naturalistic conditions. Psychology, Crime \& Law, 19(10), 893-905.

Vredeveldt, A., \& Perfect, T. J. (2014). Reduction of environmental distraction to facilitate cognitive performance. Frontiers in Psychology, 5. 
Vredeveldt, A., \& Sauer, J. D. (2015). Effects of eye-closure on confidence-accuracy relations in eyewitness testimony. Journal of Applied Research in Memory and Cognition, 4(1), 51-58.

Vredeveldt, A., Tredoux, C. G., Nortje, A., Kempen, K., Puljević, C., \& Labuschagne, G. N. (2015). A field evaluation of the Eye-Closure Interview with witnesses of serious crimes. Law and human behavior, 39(2), 189.

Willén, R. M., Granhag, P. A., Strömwall, L. A., \& Fisher, R. P. (2015). Facilitating particularization of repeated similar events with context-specific cues. Scandinavian journal of psychology, 56(1), 28-37.

Wright, A. M., \& Holliday, R. E. (2007). Interviewing cognitively impaired older adults: How useful is a Cognitive Interview?. Memory, 15(1), 17-33.

Wu, J. T., Riley, S., Fraser, C., \& Leung, G. M. (2006). Reducing the impact of the next influenza pandemic using household-based public health interventions. PLoS Med, 3(9), e361. 


\section{APPENDICES}




\section{APPENDIX A}

Contact Tracing Form for 2014 Ebola Outbreak

\section{UGANDA VIRAL HEMORRHAGIC FEVER CONTACT LISTING FORM}

\begin{tabular}{|c|c|c|c|c|c|c|c|c|c|}
\hline \multicolumn{10}{|c|}{ Case Information } \\
\hline $\begin{array}{l}\text { UVRIIMoH } \\
\text { Case ID }\end{array}$ & Surname & Other Names & Head of Household & Village & Sub-County & District & $\begin{array}{c}\text { Date of } \\
\text { Symptom } \\
\text { Onset }\end{array}$ & $\begin{array}{c}\text { Date of } \\
\text { Admission to } \\
\text { Isolation }\end{array}$ & Date of Death \\
\hline & & & & & & & & & \\
\hline
\end{tabular}

**For all information on location, please list information on where the contact will be residing for the next month.

\section{Contact Information}

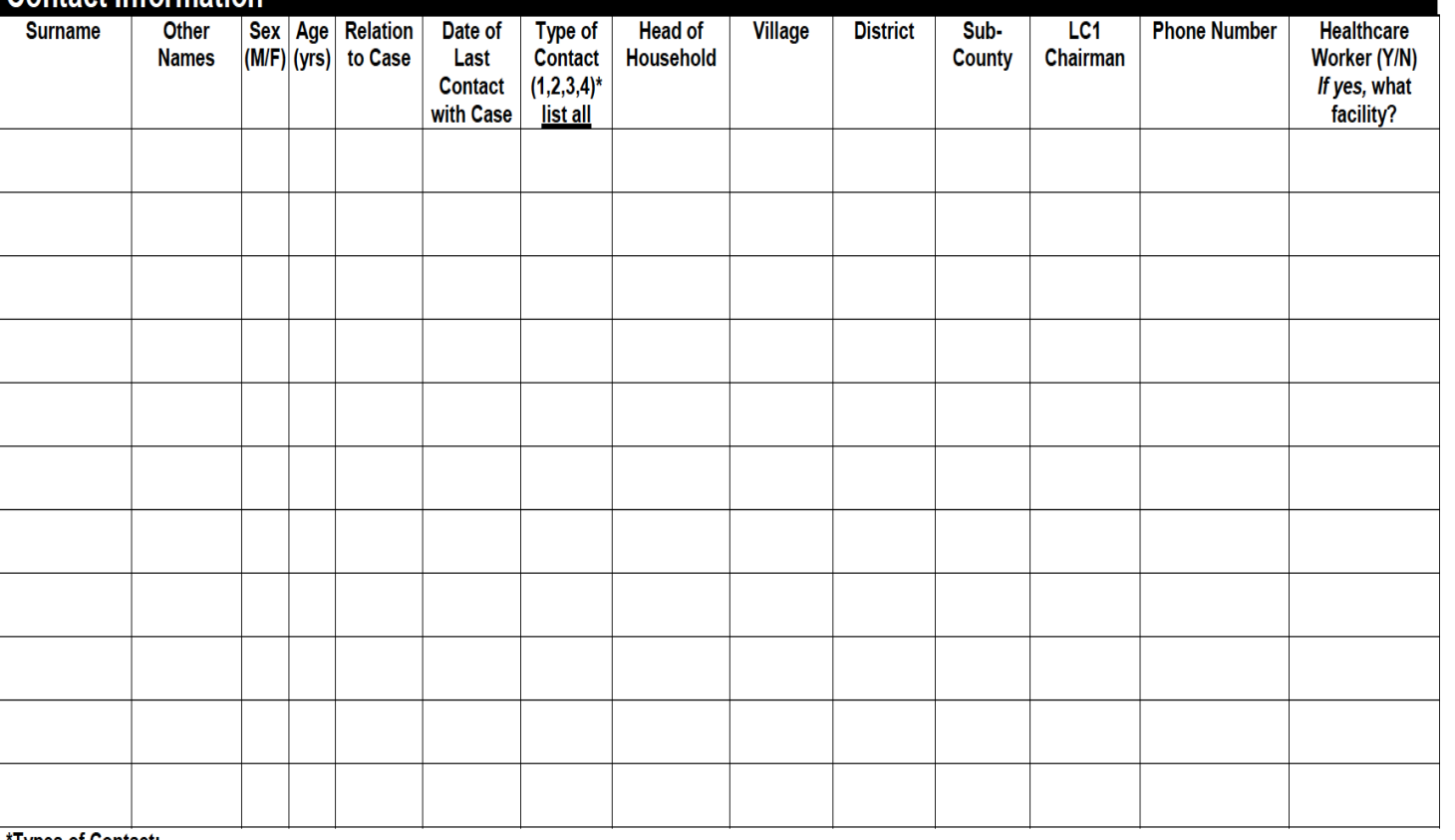

*Types of Contact:

$1=$ Touched the body fluids of the case (blood, vomit, saliva, urine, feces)

$2=$ Had direct physical contact with the body of the case (alive or dead)

$3=$ Touched or shared the linens, clothes, or dishes/eating utensils of the case

$4=$ Slept, ate, or spent time in the same household or room as the case

Contact Sheet Filled by: Name:

Position:

Phone: 


\section{APPENDIX B \\ Standard Contact Tracing Interview Protocol}

\section{Introductory Phase}

1. Introduce yourself

a. Develop rapport

i. Draw some type of connection between yourself and the person and make them feel comfortable with speaking with you

1. Example:
a. Interviewer: "Did you find parking okay today?"
b. Witness: "Yes I got here early enough"
c. Interviewer: "Good, thanks for coming in."

\section{Instruction Phase (Universal to Standard and CI)}

1. General Instructions

b. What we're going to do today is take part in a contact-tracing interview. Do you remember when the Ebola outbreak happened? Well, key to stopping the spread of diseases like Ebola is asking people who are infected to tell medical professionals who they were in contact with, so they can get the life saving treatment they need and avoid infecting others.

c. "What I need you to do now is imagine that you have been feeling ill the past 3 days and just now, with a high fever, you are coming into the hospital. The hospital tests revealed that you have the deadly infectious disease, Meningitis. It's very important to the health and safety of the public that I know about all of the individuals with whom you have had personal contact in the past 3 days. By alerting us to these individuals you could save many lives."

d. More specific instructions

i. "So, today I will be asking you about the individuals with whom you have interacted over the past 3 days (meaning from $x$ day to right now). I will be asking you to report anyone you may have touched, which includes any type of physical contact (e.g., hug, handshake, kiss) over the past 3 days. We are also interested in whether you shared a plate, or cup, straw or a cigarette (basically anything where you might have transferred germs through the mouth) with someone.

ii. We would also like to know the type of interaction you had with the person. For example, you can say 'I shook my bosses hand after my shift on Thursday'.

iii. It is also important to list the places you have visited over that time. While you provide the information, I will be filling out this form (show form). If you do not know the first and last name of a specific person, or the name of the place you were, if you provide a description of the person or place, I will make a note of it. Any 


\section{Interview Phase}

details you can provide about the person or the place, even if you do know the name, may be important in finding them

iv. Do you have any questions right now? If not, we can get started".

* Throughout the interview phase, you will fill out the attached form in which the contacts will be listed. It is important that as accurate spelling as possible is used.

So once a contact is provided, ask for the appropriate spelling to the best of the witness's knowledge.

1. Go through the "circles" of contacts (people you live with, significant other etc...)

a. "First I want you to take me through the people you've lived with at any time over the past three days.

b. Do you also have a significant other who you interacted with as specified over the past three days?

c. Do you have a job? If yes: Next, I want you to tell me about the people you may have had direct contact with at your place of employment. Over the past three days, with whom did you interact with (touch or share desk/food, drink with??) and how?

d. What about any friends you may have interacted with over the past three days?

e. Finally, tell me with whom did you interact with and how who may have been acquaintances or even a stranger over the past three days (remember: if you don't know their name, a description might help to track them down)"

2. Go through the calendar (pull out a calendar with the dates so they can visualize it), day-by-day

a. Provide a reminder about the types of contact we're looking for here

i. "Who did you interact with (as described above: touch, hand shake, shared plates etc...) on Monday?"

ii. "Who did you interact with on Tuesday?"

iii. "Who did you interact with on Wednesday?"

3. After each listing (e.g., after listing people interacted with on Monday, say, "Is there anyone else?")

4. Finish interview with, "Is there anyone else you can think of?" until the witness says, "No, that's it".

5. Thank you for participating. You will now be assigned your credit. 
APPENDIX C

Contact Tracing Form

Contact Tracing Form

Participant \#

Interviewer:

Time Slot:

Contacts During Contagion (Person and/or Place and/or Details):

\begin{tabular}{|c|c|c|c|c|}
\hline & First Name: & Last Name: & Location: & Contact Details: \\
\hline 1 & & & & \\
\hline 2 & & & & \\
\hline 3 & & & & \\
\hline 4 & & & & \\
\hline 5 & & & & \\
\hline 6 & & & & \\
\hline 7 & & & & \\
\hline 8 & & & & \\
\hline 9 & & & & \\
\hline 10 & & & & \\
\hline 11 & & & & \\
\hline 12 & & & & \\
\hline 13 & & & & \\
\hline 14 & & & & \\
\hline 15 & & & & \\
\hline 16 & & & & \\
\hline 17 & & & & \\
\hline
\end{tabular}




\section{APPENDIX D \\ Cognitive Interview Protocol}

\section{Introductory Phase}

2. Introduce yourself

a. Develop rapport

i. Draw some type of connection between yourself and the person and make them feel comfortable with speaking with you

1. Example:
a. Interviewer: "Did you find parking okay today?"
b. Witness: "Yes I got here early enough"
c. Interviewer: "Do you live close to campus?"
d. Develop some type of connection, make it clear you're comfortable also talking about your self and that this isn't a one-way interrogatory type of interview

\section{Instruction Phase (Universal to Standard and CI)}

2. General Instructions

b. What we're going to do today is take part in a contact-tracing interview. Do you remember when the Ebola outbreak happened? Well, key to stopping the spread of diseases like Ebola is asking people who are infected to tell medical professionals who they were in contact with, so they can get the life saving treatment they need and avoid infecting others.

c. "What I need you to do now is imagine that you have been feeling ill the past 3 days and just now, with a high fever, you are coming into the hospital. The hospital tests revealed that you have the deadly infectious disease, Meningitis. It's very important to the health and safety of the public that I know about all of the individuals with whom you have had personal contact in the past 3 days. By alerting us to these individuals you could save many lives."

d. More specific instructions

i. "So, today I will be asking you about the individuals with whom you have interacted over the past 3 days (meaning from $\mathrm{x}$ day to right now). I will be asking you to report anyone you may have touched, which includes any type of physical contact (e.g., hug, handshake, kiss) over the past 3 days. We are also interested in whether you shared a plate, or cup, straw or a cigarette (basically anything where you might have transferred germs through the mouth) with someone.

ii. We would also like to know the type of interaction you had with the person. For example, you can say 'I shook my bosses hand after my shift on Thursday'.

iii. It is also important to list the places you have visited over that time. While you provide the information, I will be filling out this 


\section{Interview Phase}

form (show form). If you do not know the first and last name of a specific person, or the name of the place you were, if you provide a description of the person or place, I will make a note of it. Any details you can provide about the person or the place, even if you do know the name, may be important in finding them

iv. Do you have any questions right now? If not, we can get started".

* Throughout the interview phase, you will be taking notes on the listed contacts. It's important that you can then go BACK to those contacts to ask for more specifics, like where do they live, what kind of contact was it, etc...

6. Social Dynamics

a. Steps:

i. Not like a TV interview

ii. Not going to ask a lot of questions

iii. You're the expert, you know what happened and who you have contacted with and I don't

iv. I'll just be taking notes

v. Like you're the boss and I'm the secretary

vi. Every detail is important

vii. We have a lot of time to go through this, so take you time.

7. Ideal response (very detailed, but not too long)

a. Explicitly say- this is the level of detail I want

8. Go through the first day using calendar:

a. "I want you to go through that first day. And just tell me everything you did and everyone you had contact with"

9. Go through second day:

a. Same instruction as first

10. Go through third day:

a. Same instruction as first and second

11. DO NOT EVER INTERRUPT FOR ANY REASON. Make a note and come back to it.

12. Reverse order/varied retrieval

a. Have participant close their eyes.

b. Instead of talking about what you were doing I'm going to ask you the places you were and the contacts who were there.

c. (Pause to let the first instruction sink in)

d. But I'm going to ask you to now describe the places you were on that day in reverse order.

e. So for example, from Wednesday night when you were home in bed all the way to Wednesday morning when you were home in the morning.

f. Do that for each day.

13. Context Reinstatement (pick sometime when the person was interacting with many people)

a. I want you to close your eyes and go to... (Describe the time). I want you to think about how you were feeling, what you were seeing, what you 
were smelling, and what you were doing at that time. Take a minute to develop a rich mental picture. GIVE THEM A MINUTE TO GET AN IMAGE (if they start talking quickly, tell them to take more time).

b. Tell me again what you were doing and who you interacted with.

14. Sketch

a. Pick an important scene where there were with a lot of people (depending on the person, it could be the same scene or different scene as the one chosen for context reinstatement)

b. Have them draw a sketch and NARRATE while the sketch is going on.

15. Go through the "circles" of contacts (people you live with, significant other etc...) (remind about the contacts)

a. "First I want you to take me through the people you've lived with at any time over the past three days.

b. Do you also have a significant other who you interacted with as specified over the past three days?

c. Do you have a job? If yes: Next, I want you to tell me about the people you may have had direct contact with at your place of employment. Over the past three days, with whom did you interact with (touch or share desk/food, drink with??) and how?

d. What about any friends you may have interacted with over the past three days?

e. Finally, tell me with whom did you interact with and how who may have been acquaintances or even a stranger over the past three days (remember: if you don't know their name, a description might help to track them down)"

16. Remember to ask, "what else"? or "Is there anyone else" after they say "that's it" after one of the narratives.

17. Introduce the Form.

18. Go back and ask the critical questions about each of the contacts (this is important)

19. Finish interview with, "Is there anyone else you can think of?" until the witness says, "No, that's it".

20. Thank you for participating. You will now be assigned your credit. 


\section{APPENDIX E \\ Standard Interview for Contact Tracing Cheat Sheet}

1. Develop rapport (briefly).

2. Give experiment instructions.

3. Go through circles of contacts (get spelling and additional info immediately).
a. Live with
b. Significant others
c. Job/school
d. Friends
e. Acquaintances

i. If don't know name, description will be fine

f. Is there anyone else?

4. Show calendar to aid in recall (get spelling and additional info immediately).

a. Anyone else you can think of interacting with on "x" day?

b. What about "y" day?

c. How about "z" day?

d. Anyone else you can think of?

5. Is there anyone else at all you can think of?

a. Ask this until you get a "no"

6. Thank participant for coming in and giving us this information. You will receive your credit shortly. 


\section{APPENDIX F \\ Cognitive Interview for Contact Tracing Cheat Sheet}

1. Develop rapport (draw a connection).

2. Give experiment instructions.

3. Social Dynamics
a. Not like TV
b. Not a lot of questions
c. You're the expert, you know what happened
d. I'll just be taking notes
e. Like you're the boss and I'm the secretary
f. Every detail is important
g. Take your time

4. Ideal Response
a. This is the level of detail I'd like you to provide during your interview

5. First Telling
a. Use calendar and go through every day
b. Anything else

6. PAUSE and NO interruptions.

7. Varied Retrieval (places) / Reverse Order
a. Have calendar out still
b. Ask for Places instead of what you did, and remember who you interacted with at each of those places. 
c. But, in Reverse Order (e.g., so from when you were home Wednesday night for bed to when you were home waking up Wednesday morning).

d. Ask them to close eyes to help concentrate

8. Context Reinstatement (SELECT a time for which there were many people and you want to get more information)

a. Ask them to close eyes

b. Take a minute to think back to (describe the time you want more information about, e.g., the birthday party).

c. Think about what you were feeling, and thinking, and smelling and seeing.

d. Take a minute to develop that mental image

e. (Make them wait to develop it)

f. Describe again that time and who you were in contact with.

9. Sketch (SELECT a time for which there were many people and you want to get more information)

a. Ask them to narrate what they were doing and whom they were in contact with during the sketch.

10. Circle of Contacts (Remember, no interruptions.)
a. Live with
b. Significant others
c. Job/school
d. Friends 
e. Acquaintances

i. If don't know name, description will be fine

f. Is there anyone else?

11. Fill out the form

a. Go back and ask specific questions (descriptions of contact and people) to fill out the form with all of the contacts they had reported

12. Finish interview with, "Is there anyone else you can think of?" until the witness says, "No, that's it".

13. Thank you for participating. You will now be assigned your credit. 


\author{
APPENDIX G \\ Instruction Phase (Universal to Standard and CI)
}

\title{
1. General Instructions
}

a. What we're going to do today is take part in a contact-tracing interview. Do you remember when the Ebola outbreak happened? Well, key to stopping the spread of diseases like Ebola is asking people who are infected to tell medical professionals who they were in contact with, so they can get the life saving treatment they need and avoid infecting others.

b. "What I need you to do now is imagine that you have been feeling ill the past 3 days and just now, with a high fever, you are coming into the hospital. The hospital tests revealed that you have the deadly infectious disease, Meningitis. It's very important to the health and safety of the public that I know about all of the individuals with whom you have had personal contact in the past 3 days. By alerting us to these individuals you could save many lives."

c. More specific instructions

i. "So, today I will be asking you about the individuals with whom you have interacted over the past 3 days (meaning from $\mathrm{x}$ day to right now). I will be asking you to report anyone you may have touched, which includes any type of physical contact (e.g., hug, handshake, kiss) over the past 3 days. We are also interested in whether you shared a plate, or cup, straw or a cigarette (basically anything where you might have transferred germs through the mouth) with someone.

ii. We would also like to know the type of interaction you had with the person. For example, you can say 'I shook my bosses hand after my shift on Thursday'.

iii. It is also important to list the places you have visited over that time. While you provide the information, I will be filling out this form (show form). If you do not know the first and last name of a specific person, or the name of the place you were, if you provide a description of the person or place, I will make a note of it. Any details you can provide about the person or the place, even if you do know the name, may be important in finding them

iv. Do you have any questions right now? If not, we can get started". 


\section{APPENDIX H}

Excel Sheet for Transferring Contacts from Transcripts

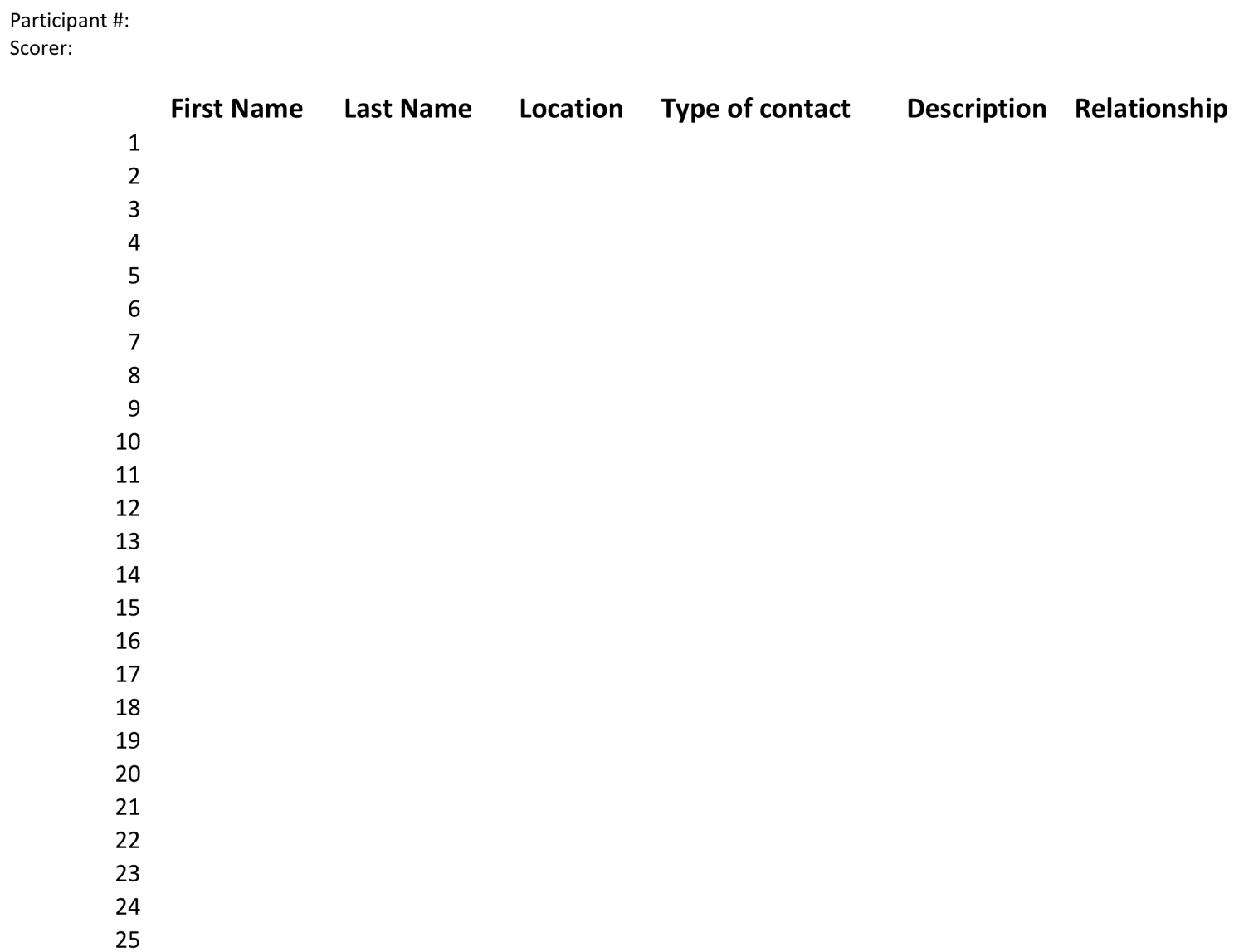




\section{APPENDIX I}

Quantity Scoring Protocol

Two Quantity Scores (Meningitis Contacts (1) and Airborne Only Contacts (2)):

\section{Meningitis Contacts (1) (physical/saliva contact only)}

If there was physical contact or saliva was shared:

Kissed

Hugged

Shared food

Shared drink

Shared eating utensils

Frequently slept in same dwelling

Lived with person (even if no contact)

\section{DOES NOT INCLUDE:}

If the person had exchanged plates at dinner (unless they ate from the same utensils) If the sick person had been handed a paper by a healthy person

If a cashier at Publix handed them their groceries

***For the above, UNLESS it's noted that their hands had touched

If the sick person and healthy person handed papers back and forth in class

If the healthy person had been in the home of the sick person, but didn't have physical contact with the sick person (the sick person was asleep while the healthy person was there, for example)

If the sick person sat next to a healthy person in class, but noted no physical contact $* * *$ Even if they sat very closely

\section{Airborne Contacts Only/Named Contacts (2)}

Count of all of the people listed- regardless of whether there was physical contact Does not include "places" (e.g., Location: FIU, Contact: No contact).

Still count when they say "no contact", because that would be a person who they would have had airborne contact with.

\section{Actual Scoring}

Under the Quantity Column in the transferred excel file, indicate the following:

Meningtis Contacts $=1$

Airborne Only Contacts $=2$ 
Places $/$ Pets $/$ Miscellaneous $=0$

When someone says "30 people" at the park, or 20 kids running around, list as one person, under the " 2 " category - since there was no contact

Score precision for all but 0

Precision score for both types of quantity:

Meningitis Contacts

Total Contacts Listed

*you would calculate these separately based on their categorization

*make a note when there is a person that they live with, but say they don't have contact with- (difference between memory and epidemiological rules) 


\section{APPENDIX J}

Red Book $29^{\text {th }}$ Edition (2012) Definition of Meningitis Contacts

\section{Table 3.36. Disease Risk for Contacts of People With Meningococcal Disease}

High risk: chemoprophylaxis recommended (close contacts)

- Household contact, especially children younger than 2 years of age

- Child care or preschool contact at any time during 7 days before onset of illness

- Direct exposure to index patient's secretions through kissing or through sharing toothbrushes or eating utensils, markers of close social contact, at any time during 7 days before onset of illness

- Mouth-to-mouth resuscitation, unprotected contact during endotracheal intubation at any time 7 days before onset of illness

- Frequently slept in same dwelling as index patient during 7 days before onset of illness

- Passengers seated directly next to the index case during airline flights lasting more than 8 hours

Low risk: chemoprophylaxis not recommended

- Casual contact: no history of direct exposure to index patient's oral secretions (eg, school or work)

- Indirect contact: only contact is with a high-risk contact, no direct contact with the index patient

- Health care personnel without direct exposure to patient's oral secretions

In outbreak or cluster

- Chemoprophylaxis for people other than people at high risk should be administered only after consultation with local public health authorities 


\section{APPENDIX K \\ Precision Scoring Protocol}

The precision of a response will be determined based on how easy a contact would be to find based on the response.

List the associated score in the precision column next to each contact.

Use the following scale.

3 (Easy to find): First and Last name

Lazaro Nunez, my friend from class at FIU

John Smith

Mother

Father

2 (Likely to find): First name and description; First name and clear relationship (e.g., coworker, friend, cousin)

John, in my psych class at FIU, he's cuban and has black hair and brown eyes

Juliette, my friends friend, at the park, she's tall and skinny, and has brown eyes

Amanda, coworker (no description)

Susan, my friend, no description

Marlene my friend at the gym (no description)

Jordan, my uncle (no description)

Kyle, Amanda's uncle (no description)

1 (Difficult to find): No name, but a description that would help to find the person (or a relationship that would help find the person); first name, but no description (and no clear relationship; e.g., at church, in class)

Waiter at Cheesecake factory who was tall, and blonde 
Girl at friend's party in Hialeah who I shared a cigarette with, she's short and has black hair

My coworker at TJ Maxx, she’s tall, blonde, and skinny

Taiwan at Mattress firm, no description

Acquaintance, dark skin, black hair, skinny

Friend, she's blonde, chubby, light skinned

Boyfriend's cousin (no description) (don't know which cousin it is)

Mother's friend (no description) (don't know which friend it is)

Aunt (no description or description; don't know which aunt it is)

Cousin (no description or description; don't know which cousin it is)

Amanda's brother, with a good description

Aunt, with a description

Cousin, with a description

Kelsey, eyebrow lady (no description)

*note: a first name and then one descriptor (e.g, male; Hispanic) constitutes a 1.

0 (Impossible to find): No name, no description, and scant ways to find the person

Kids running around at the park in Hialeah

Girl at the bar who I shared a drink with, I don't remember what she looked like

Cashier at Publix, no description

Male valet

Coworkers no description 


\section{APPENDIX L \\ Debriefing Questionnaire (No Impairment Conditions)}

1. How easy or difficult was it to your contacts?

$\begin{gathered}\text { Extremely } \\ \text { Easy }\end{gathered}$
1

2. How much mental effort/resources did you expend during the interview?

\begin{abstract}
No
Mental

Effort
\end{abstract}

1

2

3

4

3. How successfully did you remember your contacts?

Not at all successfully

1

2

3

4

5

6

Extremely

Successfully 


\section{APPENDIX M \\ Debriefing Questionnaire (Impairment Conditions)}

1. How easy or difficult was it to remember your contacts?

$\begin{gathered}\text { Extremely } \\ \text { Easy }\end{gathered}$
1

2. How much mental effort/resources did you expend during the interview?

\section{No \\ Mental \\ Effort}

Extreme

Mental

Effort

1

2

3

4

5

6

7

3. How successfully did you remember your contacts?

Not at all

Extremely successfully

1

2

3

4

5

6

Successfully

7

4. How difficult was it to complete the time estimation task during the interview?

Not at all

Effectively

1

2

4

5

Extremely

Effectively

7

5. In the space below, please indicate how much of your mental attention (If you had to choose how much of $100 \%$ of your mental effort) you feel like you allotted to the time estimation procedure and to responding during the interview (the two numbers should add up to $100 \%$ - ask RA for a calculator if needed).

Time Estimation Procedure $\%$

Interview Responses

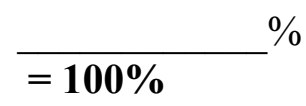




\author{
APPENDIX N \\ Experimental Protocol for Cognitive Interview with Task
}

Consent

1. Provide participant with the consent form.

2. Say, "this basically tells you that everything you say here will be anonymous and that you can leave at any time without penalty"

a. Let them read and sign

b. Make sure demos are filled out

i. FILL IN PARTICIPANT NUMBER

\title{
Training Phase (NEW!)
}

1. Today I'll be interviewing you about who you were in contact with. But, during the interview you will also be completing a simultaneous task. The task is located on my phone, here (show phone) and it is a basically a time estimation procedure. So what you will be doing is tapping the screen every time five seconds has passed. (example?)

2. It's really important that you put effort into the task. Your performance on the task is measured and it's very important you perform well on this task.

3. I'm going to ask you to practice this task for five minutes right now. Once the five minutes is up I will begin the interview.

4. Leave the room.

5. Record their score for the practice (participant number and screenshot)

6. Start new session for the interview.

\section{Introductory Phase}

3. Introduce yourself

a. Develop rapport

i. Draw some type of connection between yourself and the person and make them feel comfortable with speaking with you

1. Example:

a. Interviewer: "Did you find parking okay today?"

b. Witness: "Yes I got here early enough"

c. Interviewer: "Do you live close to campus?"

d. Develop some type of connection, make it clear you're comfortable also talking about your self and that this isn't a one-way interrogatory type of interview 


\section{Instruction Phase (Universal to Standard and CI)}

\section{General Instructions}

b. What we're going to do today is take part in a contact-tracing interview. Do you remember when the Ebola outbreak happened? Well, key to stopping the spread of diseases like Ebola is asking people who are infected to tell medical professionals who they were in contact with, so they can get the life saving treatment they need and avoid infecting others.

c. "What I need you to do now is imagine that you have been feeling ill the past 3 days and just now, with a high fever, you are coming into the hospital. The hospital tests revealed that you have the deadly infectious disease, Meningitis. It's very important to the health and safety of the public that I know about all of the individuals with whom you have had personal contact in the past 3 days. By alerting us to these individuals you could save many lives."

d. More specific instructions

i. "So, today I will be asking you about the individuals with whom you have interacted over the past 3 days (meaning from $\mathrm{x}$ day to right now). I will be asking you to report anyone you may have touched, which includes any type of physical contact (e.g., hug, handshake, kiss) over the past 3 days. We are also interested in whether you shared a plate, or cup, straw or a cigarette (basically anything where you might have transferred germs through the mouth) with someone.

ii. We would also like to know the type of interaction you had with the person. For example, you can say 'I shook my bosses hand after my shift on Thursday'.

iii. It is also important to list the places you have visited over that time. While you provide the information, I will be filling out this form (show form). If you do not know the first and last name of a specific person, or the name of the place you were, if you provide a description of the person or place, I will make a note of it. Any details you can provide about the person or the place, even if you do know the name, may be important in finding them

iv. Do you have any questions right now? If not, we can get started".

Interview Phase

* Throughout the interview phase, you will be taking notes on the listed contacts. It's important that you can then go BACK to those contacts to ask for more specifics, like where do they live, what kind of contact was it, etc... 
21. Social Dynamics

a. Steps:

i. Not like a TV interview

ii. Not going to ask a lot of questions

iii. You're the expert, you know what happened and who you have contacted with and I don't

iv. I'll just be taking notes

v. Like you're the boss and I'm the secretary

vi. Every detail is important

vii. We have a lot of time to go through this, so take you time.

22. Ideal response (very detailed, but not too long)

a. Explicitly say- this is the level of detail I want

23. Go through the first day using calendar:

a. "I want you to go through that first day. And just tell me everything you did and everyone you had contact with"

24. Go through second day:

a. Same instruction as first

25. Go through third day:

a. Same instruction as first and second

26. DO NOT EVER INTERRUPT FOR ANY REASON. Make a note and come back to it.

27. Reverse order/varied retrieval

a. Have participant close their eyes.

b. Instead of talking about what you were doing I'm going to ask you the places you were and the contacts who were there.

c. (Pause to let the first instruction sink in)

d. But I'm going to ask you to now describe the places you were on that day in reverse order.

e. So for example, from Wednesday night when you were home in bed all the way to Wednesday morning when you were home in the morning.

f. Do that for each day.

28. Context Reinstatement (pick sometime when the person was interacting with many people)

a. I want you to close your eyes and go to... (Describe the time). I want you to think about how you were feeling, what you were seeing, what you were smelling, and what you were doing at that time. Take a minute to develop a rich mental picture. GIVE THEM A MINUTE TO GET AN IMAGE (if they start talking quickly, tell them to take more time).

b. Tell me again what you were doing and who you interacted with. 
a. Pick an important scene where there were with a lot of people (depending on the person, it could be the same scene or different scene as the one chosen for context reinstatement)

b. Have them draw a sketch and NARRATE while the sketch is going on.

30. Go through the "circles" of contacts (people you live with, significant other etc...) (remind about the contacts)

a. 'First I want you to take me through the people you've lived with at any time over the past three days.

b. Do you also have a significant other who you interacted with as specified over the past three days?

c. Do you have a job? If yes: Next, I want you to tell me about the people you may have had direct contact with at your place of employment. Over the past three days, with whom did you interact with (touch or share desk/food, drink with??) and how?

d. What about any friends you may have interacted with over the past three days?

e. Finally, tell me with whom did you interact with and how who may have been acquaintances or even a stranger over the past three days (remember: if you don't know their name, a description might help to track them down)"

31. Remember to ask, "what else"? or "Is there anyone else" after they say "that's it" after one of the narratives.

32. Introduce the Form.

33. Go back and ask the critical questions about each of the contacts (this is important)

34. Finish interview with, "Is there anyone else you can think of?" until the witness says, "No, that's it".

35. Thank you for participating. You will now be assigned your credit. 
Table 1

Study 2: Means, Standard Deviations, and Confidence Intervals of Total Contacts as a Function of Interview Type and Presence of the Impairment Task

\begin{tabular}{|c|c|c|c|c|}
\hline Condition & M & SD & $\mathrm{N}$ & $95 \% \mathrm{CI}$ \\
\hline \multicolumn{5}{|c|}{ Quantity of Total Contacts } \\
\hline CI Overall & 16.78 & 7.55 & 66 & {$[15.14,18.41]$} \\
\hline Task & 15.03 & 7.32 & 35 & {$[12.79,17.27]$} \\
\hline No Task & 18.52 & 7.50 & 31 & {$[16.13,20.90]$} \\
\hline SI Overall & 12.57 & 6.02 & 72 & {$[11.01,14.14$} \\
\hline Task & 13.43 & 6.10 & 37 & {$[11.25,15.62]$} \\
\hline No Task & 11.71 & 5.91 & 35 & {$[9.47,13.96]$} \\
\hline Task Overall & 14.23 & 6.72 & 72 & {$[12.67,15.80]$} \\
\hline No Task Overall & 15.12 & 7.48 & 66 & {$[13.48,16.75]$} \\
\hline \multicolumn{5}{|c|}{ Precision of Total Contacts } \\
\hline CI Overall & 2.08 & 0.47 & 66 & {$[1.99,2.18]$} \\
\hline Task & 2.10 & 0.51 & 35 & {$[1.97,2.23]$} \\
\hline No Task & 2.06 & 0.42 & 31 & {$[1.93,2.20]$} \\
\hline SI Overall & 2.50 & 0.29 & 72 & {$[2.41,2.59]$} \\
\hline Task & 2.48 & 0.25 & 37 & {$[2.36,2.61]$} \\
\hline No Task & 2.51 & 0.33 & 35 & {$[2.38,2.64]$} \\
\hline Task Overall & 2.29 & 0.44 & 72 & {$[2.20,2.38]$} \\
\hline No Task Overall & 2.29 & 0.44 & 66 & {$[2.19,2.38]$} \\
\hline \multicolumn{5}{|c|}{ Utility of Total Contacts } \\
\hline CI Overall & 35.35 & 18.73 & 66 & {$[31.30,39.40]$} \\
\hline Task & 31.89 & 18.50 & 35 & {$[26.34,37.44]$} \\
\hline No Task & 38.81 & 18.64 & 31 & {$[32.91,44.70]$} \\
\hline SI Overall & 31.00 & 14.68 & 72 & {$[27.12,34.86]$} \\
\hline Task & 33.30 & 15.58 & 37 & {$[27.90,38.70]$} \\
\hline No Task & 28.69 & 13.47 & 35 & {$[23.14,34.24]$} \\
\hline Task Overall & 32.59 & 32.61 & 72 & {$[28.72,36.46]$} \\
\hline No Task Overall & 33.75 & 33.44 & 66 & {$[29.70,37.80]$} \\
\hline
\end{tabular}


Table 2

Study 2: Means, Standard Deviations, and Confidence Intervals of Droplet-Transmitted Contacts As a Function of Interview Type and Presence of the Impairment Task

\begin{tabular}{lcccc}
\hline Condition & $\mathrm{M}$ & $\mathrm{SD}$ & $\mathrm{N}$ & $95 \% \mathrm{CI}$ \\
\hline \multicolumn{4}{c}{ Quantity of Droplet-Transmitted Contacts } & \\
Task & 12.43 & 6.71 & 66 & {$[10.91,13.96]$} \\
No Task & 10.80 & 6.52 & 35 & {$[8.72,12.89]$} \\
SI Overall & 14.07 & 6.60 & 31 & $11.86,16.28]$ \\
Task & 10.88 & 5.99 & 72 & {$[9.43,12.33]$} \\
No Task & 12.08 & 6.34 & 37 & {$[10.06,14.11]$} \\
Task Overall & 9.60 & 5.42 & 35 & {$[7.60,11.77]$} \\
No Task Overall & 11.44 & 11.46 & 72 & {$[9.99,12.89]$} \\
& 11.88 & 11.74 & 66 & {$[10.36,13.39]$} \\
CI Overall & Precision of Droplet-Transmitted Contacts & \\
Task & 2.37 & 0.46 & 66 & {$[2.28,2.46]$} \\
No Task & 2.37 & 0.53 & 35 & {$[2.25,2.50]$} \\
SI Overall & 2.36 & 0.38 & 31 & {$[2.23,2.50]$} \\
Task & 2.67 & 0.27 & 72 & {$[2.58,2.75]$} \\
No Task & 2.61 & 0.25 & 37 & {$[2.49,2.73]$} \\
Task Overall & 2.72 & 0.28 & 35 & {$[2.59,2.86]$} \\
No Task Overall & 2.49 & 0.43 & 72 & {$[2.40,2.58]$} \\
CI Overall & 2.54 & 0.37 & 66 & {$[2.50,2.63]$} \\
Task & Utility of Droplet-Transmitted Contacts & \\
No Task & 29.50 & 16.93 & 66 & {$[25.68,33.33]$} \\
SI Overall & 25.46 & 16.75 & 35 & {$[20.22,30.70]$} \\
Task & 33.55 & 16.34 & 31 & {$[27.98,39.12]$} \\
No Task & 28.38 & 14.99 & 72 & {$[24.73,32.04]$} \\
Task Overall & 31.19 & 16.08 & 37 & {$[26.09,36.29]$} \\
No Task Overall & 29.56 & 14.99 & 35 & {$[20.33,30.81]$} \\
\hline & 25.57 & 16.55 & 72 & {$[24.67,31.98]$} \\
& 28.32 & 66 & {$[25.74,33.38]$} \\
\hline
\end{tabular}


Table 3

Study 2: Means, Standard Deviations, and Confidence Intervals of Subjective Assessments of Difficulty Remembering, Mental Resources Expended, and Success at Remembering as a Function of Interview Type and Presence of the Impairment Task

\begin{tabular}{|c|c|c|c|c|}
\hline Condition & $\mathrm{M}$ & SD & $\mathrm{N}$ & $95 \% \mathrm{CI}$ \\
\hline \multicolumn{5}{|c|}{ Difficulty Remembering } \\
\hline CI Overall & 3.75 & 1.40 & 66 & {$[3.40,4.11]$} \\
\hline Task & 3.83 & 1.36 & 35 & {$[3.34,4.31]$} \\
\hline No Task & 3.68 & 1.47 & 31 & {$[3.16,4.19]$} \\
\hline SI Overall & 3.95 & 1.60 & 71 & {$[3.60,4.29]$} \\
\hline Task & 4.57 & 1.53 & 37 & {$[4.10,5.04]$} \\
\hline No Task & 3.32 & 1.42 & 34 & {$[2.83,3.82]$} \\
\hline Task Overall & 4.23 & 1.49 & 72 & {$[3.86,4.54]$} \\
\hline No Task Overall & 3.51 & 1.45 & 65 & {$[3.14,3.86]$} \\
\hline \multicolumn{5}{|c|}{ Mental Resources Expended } \\
\hline CI Overall & 4.99 & 1.13 & 66 & {$[4.70,5.29]$} \\
\hline Task & 5.34 & 0.97 & 35 & {$[4.94,5.75]$} \\
\hline No Task & 4.65 & 1.20 & 31 & {$[4.22,5.07]$} \\
\hline SI Overall & 4.91 & 1.41 & 71 & {$[4.62,5.19]$} \\
\hline Task & 5.43 & 1.24 & 37 & {$[5.04,5.83]$} \\
\hline No Task & 4.38 & 1.39 & 34 & {$[3.97,4.79]$} \\
\hline Task Overall & 5.39 & 1.27 & 72 & {$[5.11,5.67]$} \\
\hline No Task Overall & 4.51 & 1.09 & 65 & {$[4.22,4.81]$} \\
\hline \multicolumn{5}{|c|}{ Success at Remembering } \\
\hline CI Overall & 5.23 & 1.24 & 66 & {$[4.93,5.52]$} \\
\hline Task & 5.23 & 1.09 & 35 & {$[4.82,5.64]$} \\
\hline No Task & 5.23 & 1.41 & 31 & {$[4.79,5.66]$} \\
\hline SI Overall & 5.16 & 1.29 & 71 & {$[4.88,5.45]$} \\
\hline Task & 4.65 & 1.27 & 37 & {$[4.25,5.05]$} \\
\hline No Task & 5.68 & 1.09 & 34 & {$[5.26,6.09]$} \\
\hline Task Overall & 4.94 & 1.21 & 72 & {$[4.65,5.22]$} \\
\hline No Task Overall & 5.45 & 1.26 & 65 & {$[5.15,5.75]$} \\
\hline
\end{tabular}

Note: Anchors for measures are as follows: difficulty remembering ( 1 being extremely easy and 7 being extremely difficult, mental resources expended ( 1 being no mental effort and 7 being extreme mental effort), success at remembering ( 1 being not at all successfully and 7 being extremely successfully). 


\section{Table 4}

Study 2: Means, Standard Deviations, and Confidence Intervals of Subjective Assessments of Difficulty Completing Task, and Percent of Mental Resources Allocated to the Task as a Function of Interview Type

\begin{tabular}{lcccc}
\hline Condition & $\mathrm{M}$ & $\mathrm{SD}$ & $\mathrm{N}$ & $95 \%$ CI \\
\hline & \multicolumn{5}{c}{ Difficulty Completing the Task } \\
CI Task & 5.17 & 1.50 & 35 & {$[4.64,5.70]$} \\
SI Task & 5.22 & 1.64 & 36 & {$[4.70,2.75]$} \\
\multicolumn{5}{c}{ Percent of Resources Allocated to the Task } \\
CI Task & 43.23 & 14.37 & 35 & {$[37.43,49.03]$} \\
SI Task & 48.42 & 10.55 & 36 & {$[42.70,54.13]$} \\
\hline
\end{tabular}

Note: Anchors for measures are as follows: difficulty completing task ( 1 being not at all effectively and 7 being extremely effectively), percent of mental resources allocated to task (out of 100). 
Table 5

Study 2: Means, Standard Deviations, and Confidence Intervals of Length of Interview as a Function of Interview Type and Presence of the Impairment Task

\begin{tabular}{lcccc}
\hline & \multicolumn{4}{c}{ Interview Length } \\
\cline { 2 - 5 } Condition & $\mathrm{M}$ & $\mathrm{SD}$ & $\mathrm{N}$ & $95 \%$ CI \\
\hline CI Overall & 40.49 & 11.44 & 66 & {$[38.38,42.60]$} \\
Task & 39.68 & 10.04 & 35 & {$[36.79,42.57]$} \\
No Task & 41.30 & 12.95 & 31 & {$[36.79,42.57]$} \\
SI Overall & 19.58 & 6.44 & 72 & {$[17.56,21.59]$} \\
Task & 24.00 & 4.82 & 37 & {$[21.19,26.81]$} \\
No Task & 15.15 & 4.55 & 35 & {$[12.27,18.04]$} \\
Task Overall & 31.84 & 11.06 & 72 & {$[29.83,33.86]$} \\
No Task Overall & 28.23 & 16.16 & 66 & {$[26.12,30.33]$} \\
\hline
\end{tabular}




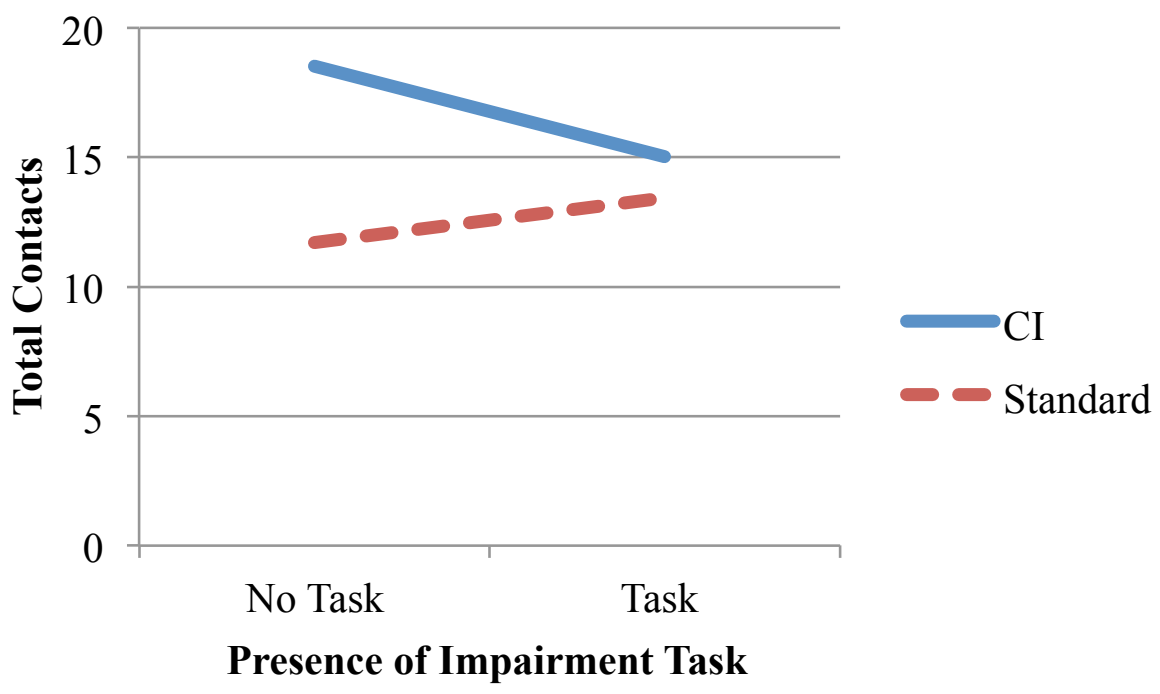

Figure 1. Study 2: Total Contacts listed as a function of interview type and presence of impairment task. 


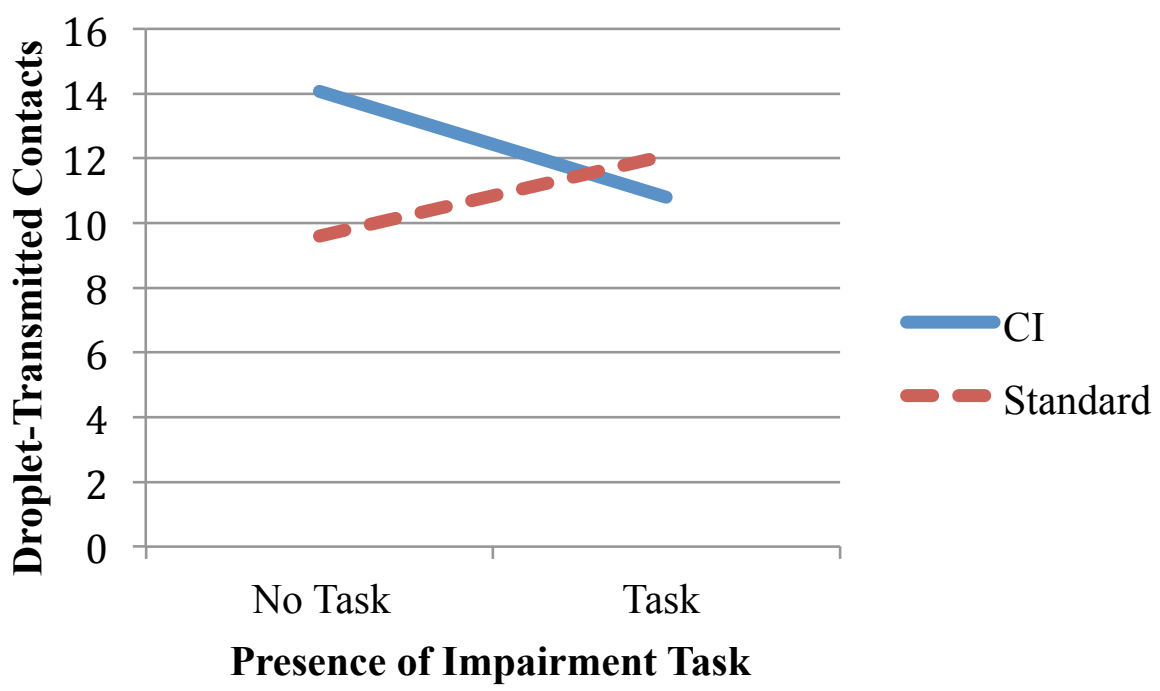

Figure 2. Study 2: Droplet-transmitted contacts listed as a function of interview type and presence of impairment task. 


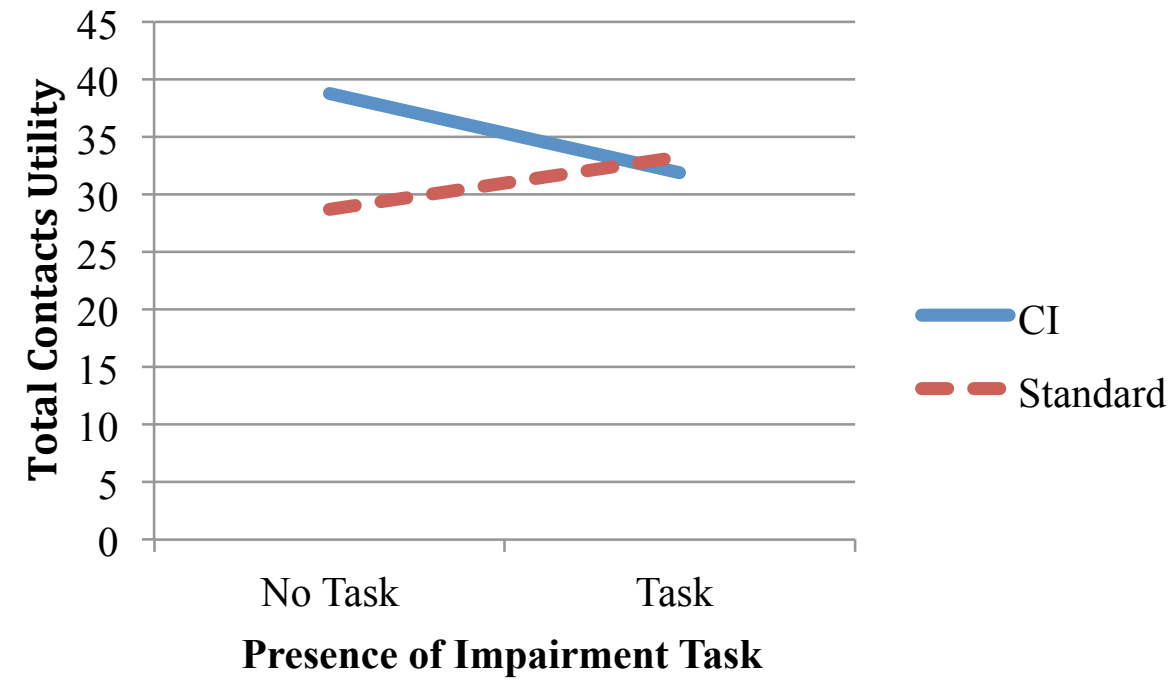

Figure 3. Study 2: Utility of total contacts as a function of interview type and presence of impairment task. 


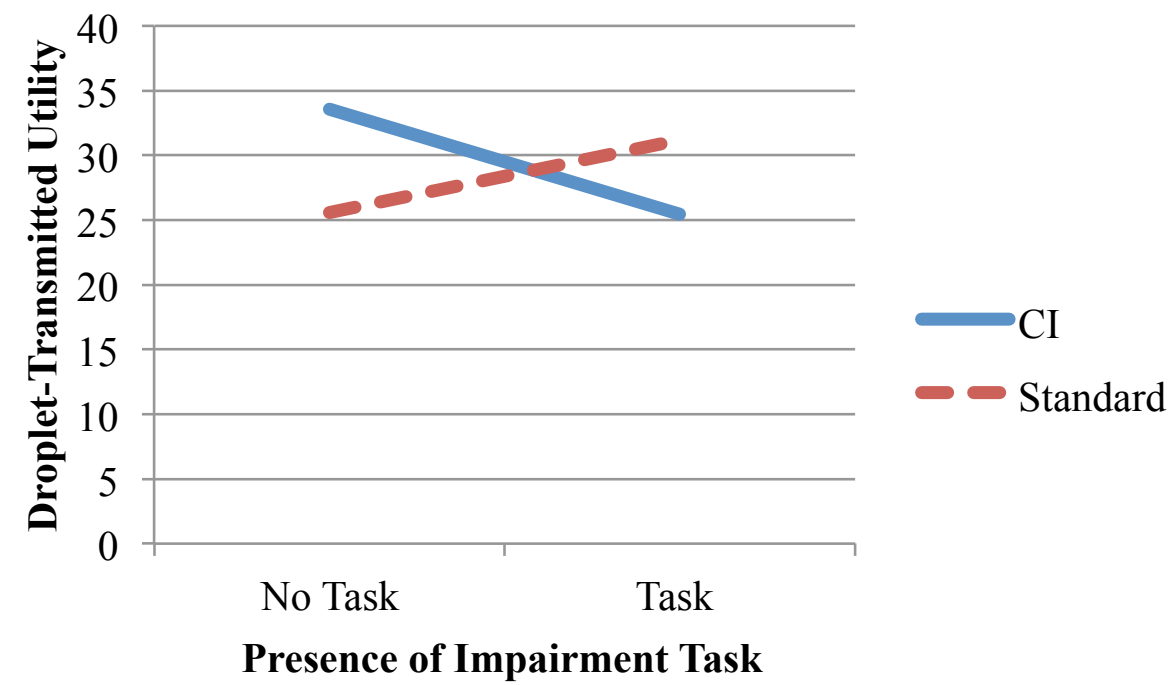

Figure 4. Study 2: Utility of droplet-transmitted contacts as a function of interview type and presence of impairment task. 


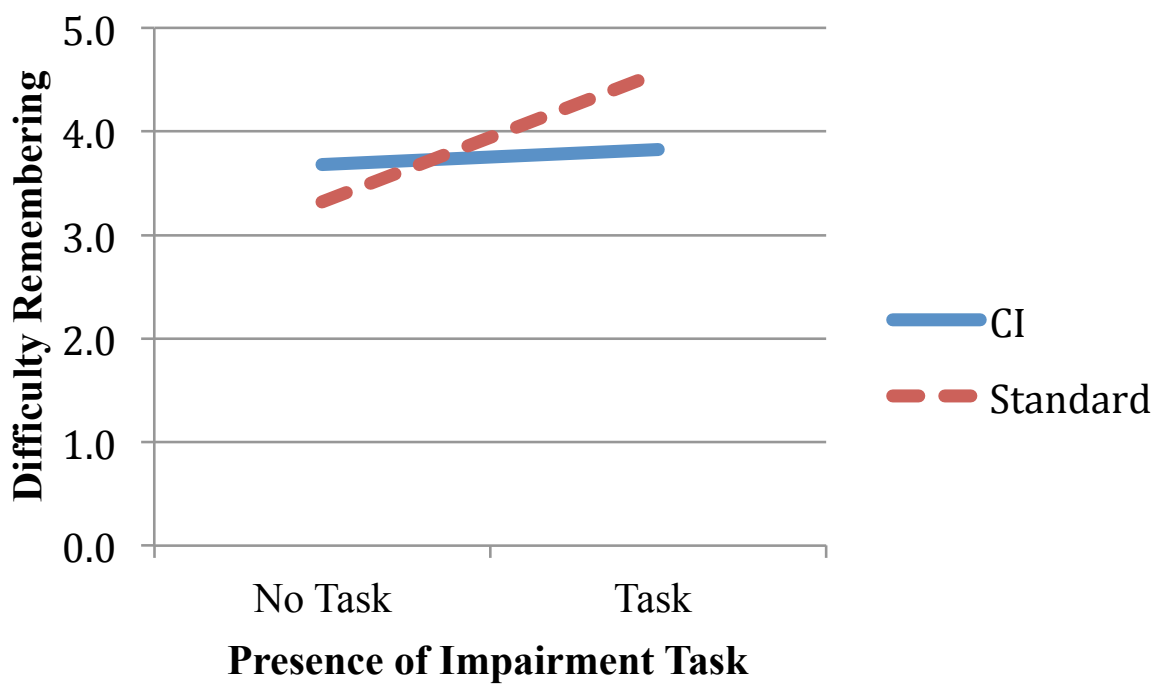

Figure 5. Study 2: Ratings of difficulty remembering as a function of interview type and presence of impairment task (higher score indicates greater difficulty). 


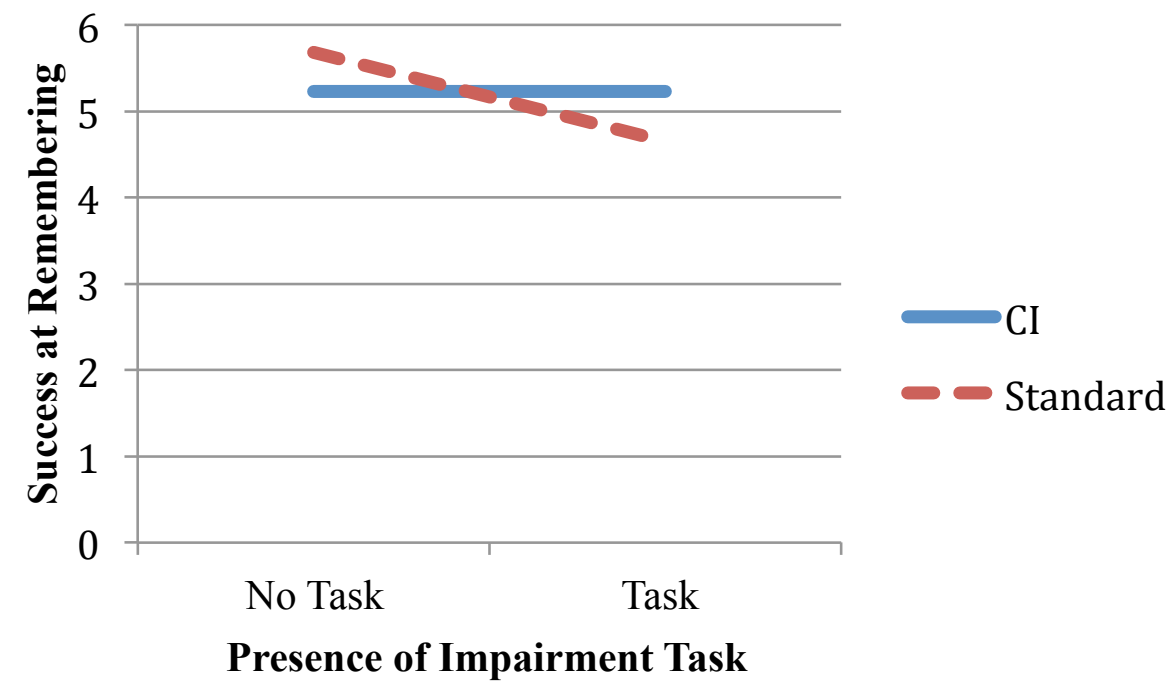

Figure 6. Study 2. Ratings of success of remembering contacts as a function of interview type and presence of impairment task (higher scores indicate greater success). 


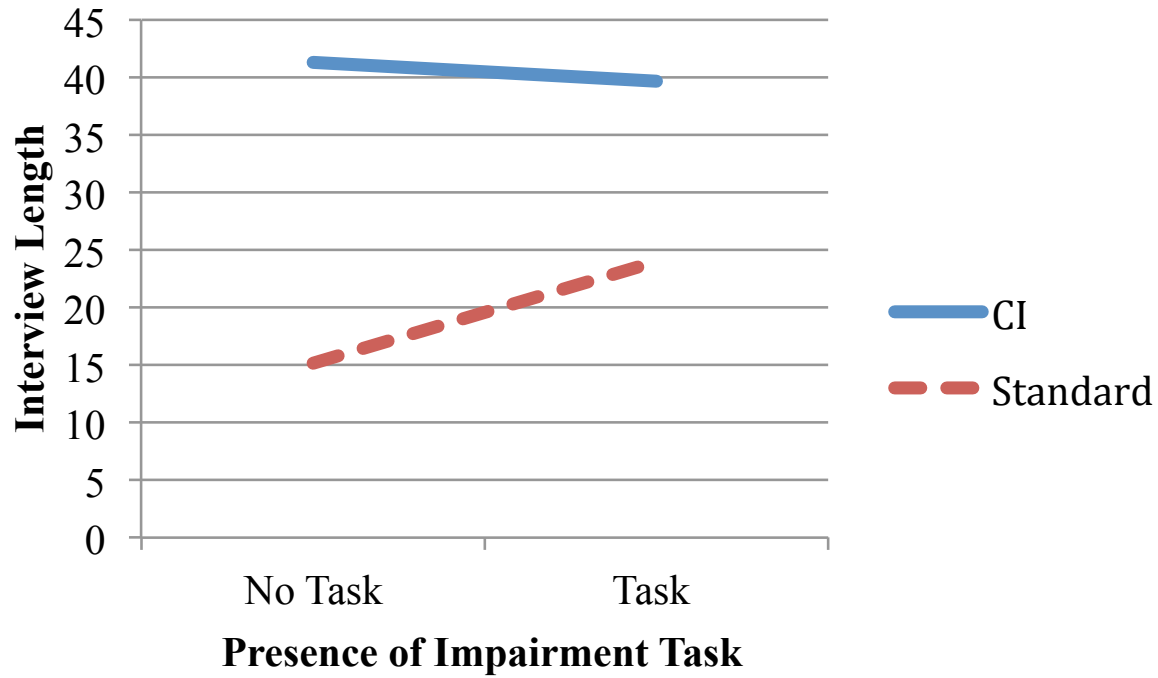

Figure 7. Study 2: Interview length as a function of interview type and presence of impairment task. 
VITA

\section{ALEXANDRA E. MOSSER}

April 2012

B.S., Psychology and Sociology

University of Pittsburgh

Pittsburgh, Pennsylvania

December $2014 \quad$ M.S., Psychology

Florida International University

Miami, Florida

July 2015 Doctoral Candidate

Florida International University

Miami, Florida

\section{PUBLICATIONS AND PRESENTATIONS}

Mosser, A. E., Evans, J. R., Boza, Z., Nunez, L., Padron, S., Weber, C. (2017).

Expanding the frontiers of investigative interviewing: Using the Cognitive Interview to enhance infectious disease contact tracing. Invited talk presented at the annual Southern Society of Philosophers and Psychologists conference, Savannah, GA. Presented by Mosser, A. E.

Mosser, A. E., Evans, J. R., Boza, Z., Nunez, L., Padron, S., Weber, C. (2017). From the police station to the hospital bed: Applying the Cognitive Interview to infectious disease contact tracing. Paper presented at the annual American Psychology-Law Society conference, Seattle, WA. Presented by Mosser, A. E.

Mosser, A. E., Fisher, R. P., Manon, M. (2017). Interviewing witnesses twice: Using a double-interview paradigm to assess the accuracy and veracity of witness recollection. Paper presented at the annual American Psychology-Law Society conference, Seattle, WA. Presented by Mosser, A. E.

Mosser, A. E., Ertzinger, B. (2016). Eliciting information from memory via the Cognitive Interview. Invited talk presented at the High Value Detainee Interrogation Group Presentation to Practitioners, Washington, D.C. Presented by Mosser, A. E.

Mosser, A. E., Fisher, R. P., Manon, M. (2016). Interviewing information sources twice with the Cognitive Interview: Novel measures to assess the accuracy and veracity of witness recollection. Paper presented at the High Value Detainee Interrogation Group Research Symposium, Washington, D.C. Presented by Mosser, A. E.

Mosser, A. E., Evans, J. R., Fisher, R. P. (2016). Enhancing recall during contact tracing using the Cognitive Interview. Paper presented at Fishchrift: Applied Cognition and the Cognitive Interview, Miami, FL. Presented by Mosser, A. E. 
Hagger, M. S., Chatzisarantis, N. L. D., Alberts, H., Anggono, C. O., Birt, A., Brand, R., ... Mosser, A. E., ... Zweinenberg, M. (2016). A Multi-Lab Pre-Registered Replication of the Ego-Depletion Effect. Perspectives on Psychological Science, 11, 546-573.

Mosser, A. E., Fisher, R.P., Molinaro, P., Satin, G., Manon, M. (2016). Train-theTrainer: Training law enforcement investigators in the Cognitive Interview. Paper presented at the annual American Psychology-Law Society conference, Atlanta, GA. Presented by Mosser, A. E.

Fisher, R. P., Mosser, A. E., \& Satin, G.E. (2015). Inconsistent Witness Testimony. In A. Reilly \& Pass, A. (Eds) Forensic Science. VOL 2. Grey House Publishing: Amenia, NY. Pages 590-92.

Fisher, R. P., Mosser, A.E., Molinaro, P., \& Satin, G.E. (2015). Cognitive Interview Techniques. In A. Reilly \& Pass, A. (Eds) Forensic Science. VOL 1. Grey House Publishing: Amenia, NY. Pages 224-227.

Mosser, A. E., Fisher, R.P., Molinaro, P., Satin, G., Manon, M. (2015). Training the trainer on the Cognitive Interview. Invited talk presented at the High Value Detainee Interrogation Group Research Symposium, Washington D.C.

Molinaro, P., Fisher, R. P., Mosser, A. E., Satin, G., Robertson, B. (2015). Training law enforcement trainers on the Cognitive Interview. Paper presented at the annual American Psychology-Law Society conference, San Diego, CA. Presented by Mosser, A. E.

Mosser, A. E., Fisher, R. P., Rose, L. (2014). Does inconsistency predict accuracy?: Investigating the inconsistency-accuracy relationship with guidance from a litigation expert. Talk presented at the American Psychology-Law Society Annual conference, New Orleans, LA. Presented by Mosser, A. E.

Evans, J., Michael, S., Mosser, A. E., Allen, A., Tran, H. (2014). The impact of interviewee ego depletion on information Quality. Poster presented at the American Psychology-Law Society Annual Conference, New Orleans, LA.

Molinaro, P., Fisher, R. P., Mosser, A. E., Satin, G. (2014). Training the trainer on the Cognitive Interview: Translating science into practice. Invited talk presented at the High Value Detainee Interrogation Group Presentation to Practitioners, Washington, D.C.

Molinaro, P., Fisher, R. P., Mosser, A. E., Satin, G., Robertson, B. (2014). Training law enforcement trainers on the Cognitive Interview. Invited talk presented at the High Value Detainee Interrogation Group Research Symposium, Washington, D.C.

Mosser, A., Frieze, I. H. (2012). Work plans and Gender-Role Attitudes of Traditional Women, Non-Traditional Women and Men. Journal of Behavioural Sciences, 22(3),1-17. 